\title{
THE CONCEPT OF A SIMPLE POINT OF AN ABSTRACT ALGEBRAIC VARIETY
}

\author{
BY \\ OSCAR ZARISKI
}

Contents

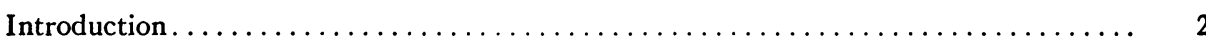

PART I. The local theory

1. Notation and terminology $\ldots \ldots \ldots \ldots \ldots \ldots \ldots \ldots \ldots \ldots \ldots \ldots \ldots \ldots, 5$

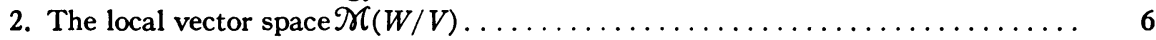

2.1. The mapping $\mathfrak{m} \rightarrow \mathfrak{m} / \mathfrak{m}^{2} \ldots \ldots \ldots \ldots \ldots \ldots \ldots \ldots \ldots \ldots \ldots \ldots \ldots \ldots, 6$

2.2. Reduction to dimension zero $\ldots \ldots \ldots \ldots \ldots \ldots \ldots \ldots \ldots \ldots \ldots \ldots \ldots \ldots \ldots \ldots$

2.3. The linear transformation $\mathcal{X}(W / V) \rightarrow \mathcal{X}\left(W / V^{\prime}\right) \ldots \ldots \ldots \ldots \ldots \ldots \ldots, 8$

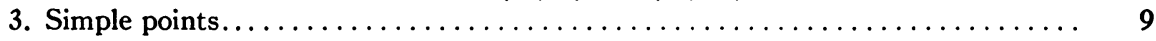

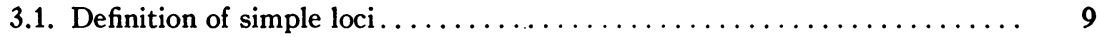

3.2. Geometric aspects of the definition....................... 10

3.3. Local ideal bases at a simple point $\ldots \ldots \ldots \ldots \ldots \ldots \ldots \ldots \ldots \ldots, 12$

3.4. Simple zeros of ideals $\ldots \ldots \ldots \ldots \ldots \ldots \ldots \ldots \ldots \ldots \ldots \ldots \ldots \ldots \ldots, 14$

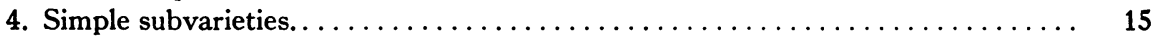

4.1. Generalization of the preceding results $\ldots \ldots \ldots \ldots \ldots \ldots \ldots \ldots \ldots, 15$

4.2. Singular subvarieties and singular points $\ldots \ldots \ldots \ldots \ldots \ldots \ldots \ldots, 16$

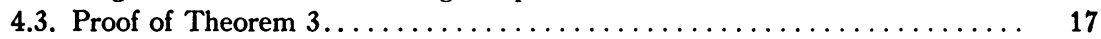

4.4. Continuation of the proof $\ldots \ldots \ldots \ldots \ldots \ldots \ldots \ldots \ldots \ldots \ldots \ldots, 17$

4.5. The case of a reducible variety $\ldots \ldots \ldots \ldots \ldots \ldots \ldots \ldots \ldots \ldots, \quad 19$

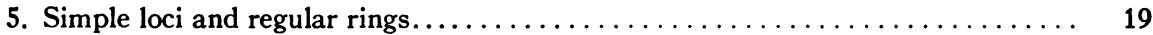

5.1. The identity of the two concepts....................... 19

5.2. Unique local factorization at simple loci .................. 22

5.3. The abstract analogue of Theorem 3 and an example of F. K. Schmidt... 23

PART II. Jacobian criteria for simple loci

6. The vector space $\mathscr{D}(W)$ of local differentials..................... 25

6.1. The local $W$-differentials in $S_{n} \ldots \ldots \ldots \ldots \ldots \ldots \ldots \ldots \ldots \ldots \ldots, \quad 25$

6.2. The linear transformation $\mathscr{X}\left(W / S_{n}\right) \rightarrow \mathscr{D}(W) \ldots \ldots \ldots \ldots \ldots \ldots \ldots .25$

7. Jacobian criterion for simple points: the separable case $\ldots \ldots \ldots \ldots \ldots \ldots \ldots, 26$

7.1. Criterion for uniformizing parameters $\ldots \ldots \ldots \ldots \ldots \ldots \ldots \ldots \ldots, \quad 26$

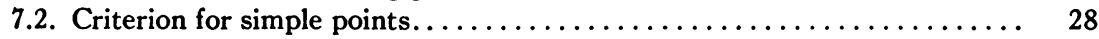

8. Continuation of the separable case: generalization to higher varieties in $S_{n} \ldots \ldots \quad 28$

8.1. Extension of the preceding results. $\ldots \ldots \ldots \ldots \ldots \ldots \ldots \ldots \ldots \ldots, 28$

8.2. A proof of the theorem of MacLane........................ 29

8.3. The singular manifold of an algebraic variety $\ldots \ldots \ldots \ldots \ldots \ldots \ldots, \quad 30$

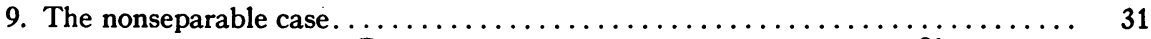

9.1. The dimension of $\mathcal{D}(W)$ as a numerical character of the field $\mathcal{f}(W) \ldots \ldots \quad 31$

9.2. Abstract differentiations over $\kappa^{p} \ldots \ldots \ldots \ldots \ldots \ldots \ldots \ldots \ldots \ldots, \quad 33$

9.3. A lemma on the canonical uniformizing parameters.............. 34

9.4. The vector space $\mathcal{D}^{*}(W)$ of the mixed local $W$-differentials.......... 36

9.5. The nonsingular character of the linear transformation $\mathcal{X}\left(W / S_{n}\right) \rightarrow \mathcal{D}^{*}(W)$. $\quad 37$

9.6. General Jacobian criteria for uniformizing parameters and for simple loci. . $\quad 38$

Presented to the Society, August 23, 1946; received by the editors August 15, 1946. 
10. Absolutely simple loci.................................. 39

10.1. Algebraic ground field extensions. . . . . . . . . . . . . . 39

10.2. Jacobian criterion for absolutely simple loci................ 41

11. Intrinsic characterizations of absolutely simple loci............... 43

11.1. Differential characterization ........................ 43

11.2. Direct verification of the local character of the definition.......... 44

11.3. André Weil's criterion.............................. 45

11.4. A criterion of analytical equivalence .................... 49

\section{INTRODUCTION}

In [11] and $[12]\left({ }^{1}\right)$ we have developed the concept of a simple point of an algebraic variety over an arbitrary ground field of characteristic zero. Our analysis of this concept has led us to the following characteristic property of a simple point which was novel and of intrinsic character (from the standpoint of regular birational transformations), since it referred to the quotient ring of the point and did not involve the ambient space of the variety:

A. A point $P$ of an $r$-dimensional irreducible algebraic variety $V$ is a simple point of $V$ if and only if the ideal of nonunits in the quotient ring of $P$ has a basis of $r$ elements.

This property of a simple point played an essential role in our study of other questions, for instance in the problem of local uniformization, and for this reason we found it convenient to use $\mathrm{A}$ as a definition of simple points $\left({ }^{2}\right)$.

On the other hand we have the following classical and time-honored definition of simple points:

B. If $\left(\left(f_{1}\left(x_{1}, x_{2}, \cdots, x_{n}\right), f_{2}\left(x_{1}, x_{2}, \cdots, x_{n}\right), \cdots, f_{v}\left(x_{1}, x_{2}, \cdots, x_{n}\right)\right)\right.$ is a basis of the defining prime ideal of an r-dimensional irreducible algebraic variety $V$ in an $S_{n}$, then a point $P$ of $V$ is simple for $V$ if and only if the Jacobian matrix $\partial\left(f_{1}, f_{2}, \cdots, f_{v}\right) / \partial\left(x_{1}, x_{2}, \cdots, x_{n}\right)$ is of rank $n-r$ at $P$.

If the ground field $\kappa$ is of characteristic zero or a perfect field of characteristic $p \neq 0$, the two definitions $\mathrm{A}$ and $\mathrm{B}$ are equivalent (see $\$ 7.2$, Theorem 7 and corollary), but in the case of nonperfect fields the two definitions may very well cease to be equivalent. We illustrate this fact by some examples.

Example 1. If $a$ is an element of $\kappa$ such that $a^{1 / p} \notin \kappa$, then the polynomial $f(x, y)=x^{p}+y^{p}-a$ is irreducible over $\kappa(p=$ characteristic of $\kappa, p \neq 0)$. The partial derivatives $\partial f / \partial x, \partial f / \partial y$ vanish identically; therefore in the sense of Definition $\mathrm{B}$ all the points of the irreducible curve $f(x, y)=0$ are singular. On the other hand it is easily seen that in the sense of Definition A all the points of the curve are simple. To see this one either shows directly that in the quotient ring of any point of the curve the nonunits form a principal ideal, or one observes that we are dealing here with a normal curve, since the coördinate ring $\kappa[\xi, \eta]$ (where $\xi$ and $\eta$ are the coördinates of the general point of the

(1) Numbers in brackets refer to the references cited at the end of the paper.

(2) See [12, p. 199]. This definition is restated in the present paper in terms of vector spaces (Definition 1, \$3.1). 
curve) is integrally closed $\left({ }^{3}\right)$.

Example 2. The field $\kappa(\xi, \eta)$ of rational functions on the curve of the preceding example is not separably generated over $\kappa$. Now, quite generally, let $V$ be an irreducible algebraic variety over $\kappa$ and let $\mathcal{f}(V)$ denote the field of rational functions on $V$. It can be shown that if $\mathcal{F}(V)$ is not separably generated over $\kappa$ then all the points of $V$ are singular in the sense of Definition B (see §8.1). On the other hand we prove (Theorem 3 and corollary, \$4.2) that every irreducible variety carries points which are simple in the sense of Definition A.

Example 3. Our attention has been called by Chevalley to the following example: $f(x, y)=y^{2}+x^{p}-a=0, p \neq 2, \alpha=a^{1 / p} \bigoplus_{\kappa}$. We have $\partial f / \partial x=0, \partial f / \partial y=2 y$, and hence the curve has one singular point $(\alpha, 0)$ in the sense of Definition B. Again we are dealing here with a normal curve, and hence, according to Definition A, all the points of the curve are simple. This example is significant because not only is the field of functions on the given curve separably generated over $\kappa$, but the curve is even absolutely irreducible (that is, the polynomial $y^{2}+x^{p}-a$ remains irreducible upon any extension of the ground field).

In $\$ 7.2$ we shall prove (Theorem 7 ) that points which are simple in the sense of Definition B are also simple in the sense of Definition A. There is ample evidence in the present paper, as well as in previous papers of ours, in support of the thesis that it is the more general concept of a simple point, as defined in $\mathrm{A}$, that constitutes the natural generalization of the classical concept of simple point. The considerations expounded below will serve the twofold purpose of reviewing this evidence and of clarifying the underlying ideas and the motivation of our present systematic treatment of the general concept of a simple point in algebraic geometry.

(a) The case of algebraic curves is particularly illuminating and deserves special consideration. A normal algebraic curve $C$ is a true projective model of the Riemann surface of the field $\mathcal{F}(C)$ (in the sense of Dedekind-Weber), for in the first place the points of such a curve are in 1-1 correspondence with the prime divisors of the field $\mathcal{F}(C)$, and in the second place the quotient ring of any point $P$ of $C$ coincides with the set of functions in $\mathcal{f}(C)$ which have non-negative order at the corresponding divisor $\mathfrak{p}$ (in other words: that quotient ring is a valuation ring). Now as long as we remain within the field $\mathcal{F}(C)$ there is no good reason why certain prime divisors of this field be called singular, and there is even less reason why all the prime divisors of $\mathcal{F}(C)$ be regarded as singular whenever $\mathcal{F}(C)$ is not separably generated over $\kappa$. From this point of view it would appear that no point of a normal curve deserves to be branded as singular.

(b) On the other hand it must be observed that the curves of Examples 1 and 3 do have at their "singular" points (in the sense of Definition B) a singular behavior with respect to suitable extensions of the ground field $\kappa$. If we

(3) For the concept of a normal variety see [11], especially Theorem $11^{\prime}$, and $[13$, p. 506]. 
pass to the field $\kappa_{1}=\kappa(\alpha)$, then the curve of Example 1 becomes a $p$-fold line: $x^{p}+y^{p}-a=(x+y-\alpha)^{p}=0$, and therefore all the points of the given curve become $p$-fold points upon the ground field extension $\kappa \rightarrow \kappa_{1}$. A similar situation prevails at the point $(\alpha, 0)$ of the curve of Example 3, and this time the phenomenon is even more apparent because of the absolute irreducibility of the curve: the curve $y^{2}+x^{p}-a=0$ remains irreducible over $\kappa_{1}$, but since its equation takes the form $y^{2}+(x-\alpha)^{p}=0$ it follows that the point $(\alpha, 0)$ is singular, in the sense of Definition A, if viewed from the level of the new ground field $\kappa_{1}$. These examples show that points which are simple in the sense of Definition A may become singular when the ground field is extended. We prove in $\$ 10.2$ (Theorem 13) that a point which is simple in the sense of Definition A is also simple in the sense of Definition B if and only if it remains simple under any extension of the ground field. For this reason we use the term "absolutely simple" (proposed by André Weil) to designate points which are simple in the sense of Definition B (see Definition 2, \$10.2). From now on we shall use the term "simple" in the sense of Definition A.

(c) We have proved in [13] that simple points behave under birational transformations in the abstract case in much the same way as they do in the classical case. In [14] we have extended to arbitrary ground fields the well known theorem of Bertini on the variable singular points of a linear system (whereas the old proofs of this theorem invariably make use of the usual differential conditions for simple points). Thus we have two significant instances of classical questions which involve the concept of a simple point and in which it turns out that the final results have nothing to do with the Jacobian criterion B. Our work on the problems of local uniformization and the resolution of singularities contains a considerable amount of material which remains valid for ground fields of characteristic $p \neq 0$, provided simple points are intended in the sense of Definition $A$. The final results in these problems are definitely false if by "simple" we mean "absolutely simple" (see Example 2 above). That the difficulties still to be overcome in the case $p \neq 0$ are merely of a technical nature and are not caused by any flaw in our formulation of the general concept of a simple point is strongly indicated by the fact that these difficulties already arise in the case of perfect ground fields when the two definitions $\mathrm{A}$ and $\mathrm{B}$ are, as we know, still equivalent.

(d) The Jacobian criterion for absolutely simple points implies that the points of an algebraic variety $V$ which are not absolutely simple are those which satisfy a certain system of algebraic equations. Hence these points form an algebraic manifold, just as in the classical case the singular manifold is always algebraic. The only difference is that in the case of nonperfect fields the points which are not absolutely simple may fill up the entire variety $V$. In this respect the concept of an absolutely simple point presents no new problem.

We face an entirely different situation when we deal with the general con- 
cept of a simple point, since Definition A is strictly of a local character; it does not give a global criterion which would characterize singular points by means of algebraic equations. Therefore in the case of nonperfect fields two questions can be formulated: (1) is the singular manifold of an algebraic variety $V$ algebraic? (2) is this manifold always a proper subset of $V$ ? The present investigation originated in these two questions, and we answer both in the affirmative. The second question can be settled already by local considerations. That is done in $\$ 4$ of Part I (see Theorem 3 and corollary in $\$ 4.2$ ). In Part I we study the concept of a simple point by local methods only and we go as far as those methods permit.

Much more difficult is the proof that the singular manifold is algebraic. The proof is achieved by deriving an algebraic criterion for simple points in which there occur certain mixed Jacobian matrices (see Theorem 11 and corollary, §9.6). In these matrices there appear derivatives of two types: (1) ordinary derivatives with respect to the variable coördinates $x_{1}, x_{2}, \cdots, x_{n}$; (2) derivatives which arise from abstract differentiations in $\kappa$ over $\kappa^{p}$. Our general Jacobian criterion reduces of course to the classical criterion B if $\kappa$ is a perfect field.

Although so far we spoke only of points, actually this paper deals with subvarieties of any dimension of a given variety $V$. In principle, by a well known reduction to the zero-dimensional case ( $\$ 2.2)$, the case of higher subvarieties should not present new features, but in point of fact it does lead to specific results which are of interest. For an indication of the nature of these results, as well as of other results not mentioned in this introduction, we refer the reader to the table of contents.

It is with pleasure that the author takes this opportunity of gratefully acknowledging the stimulating discussions and the lively correspondence which he has had with André Weil and in the course of which the ideas embodied in the present work gradually took shape in his mind.

\section{PART I. The LOCAL THEORY}

1. Notation and terminology. We fix an arbitrary abstract field $\kappa$ and we refer to it as ground field (or field of coefficients). The algebraically closed field determined by $\kappa$ shall be denoted by $\bar{\kappa}$ and shall be referred to as the field of constants.

A point $P$ is an ordered $n$-tuple $\left(\alpha_{1}, \alpha_{2}, \cdots, \alpha_{n}\right)$ of constants $\alpha_{i}$ (that is, $\left.\alpha_{i} \in \bar{\kappa}\right)$. However, we stipulate that two $n$-tuples $(\alpha)$ and $(\beta)$ represent one and the same point (over $\kappa$ ) if (and only if) they are conjugate over $\kappa$, that is, if there exists a $\kappa$-isomorphism of the field $\kappa\left(\alpha_{1}, \alpha_{2}, \cdots, \alpha_{n}\right)$ onto the field $\kappa\left(\beta_{1}, \beta_{2}, \cdots, \beta_{n}\right)$ in which to $\alpha_{i}$ there corresponds $\beta_{i}(i=1,2, \cdots, n)$. Hence a point (over $\kappa$ ), if viewed from the level of the field $\bar{\kappa}$, is actually a complete set of conjugate points with respect to $\kappa$. The totality of all points is called the linear $n$-dimensional space over $\kappa$ and is denoted by $S_{n}^{\kappa}$. The superscript indi- 
cating the field will be used only if a field other than $\kappa$ is used temporarily as ground field. Consequently, in the case of the given ground field $k$ we shall write $S_{n}$ instead of $S_{n}^{\kappa}$.

If $x_{1}, x_{2}, \cdots, x_{n}$ are indeterminates, an ideal $\mathfrak{A}$ in the polynomial ring $\kappa\left[x_{1}, x_{2}, \cdots, x_{n}\right]$ defines an algebraic variety $V=V / \kappa$ in $S_{n}$ over $\kappa$, the zero manifold of $\mathfrak{A}$. We shall denote this variety by $U(\mathfrak{H})$. Conversely, every algebraic variety $V$ defines uniquely an ideal, namely the ideal consisting of those polynomials $f(x)$ which vanish at every point of $V$. We shall denote this ideal by $I(V)$. If $V=\mathcal{V}(\mathfrak{A})$, then $I(V)=$ Radical of $\mathfrak{A}$ (Hilbert's Nullstellensatz) [15] and $U(I(V))=V$.

For an irreducible variety $V$ the ideal $I(V)$ is prime and shall be denoted by $p(V)$. If $p=p(V)$ and if $\xi_{i}$ denotes the $p$-residue of $x_{i}$, then the ordered $n$-tuple $\left(\xi_{1}, \xi_{2}, \cdots, \xi_{n}\right)$ is a general point of $V$. We mean by the coördinate ring of $V$ the ring $\kappa\left[\xi_{1}, \xi_{2}, \cdots, \xi_{n}\right]$, and we denote this ring by $R[V]$. Similarly we denote by $\mathcal{F}(V)$ the field $\kappa\left(\xi_{1}, \xi_{2}, \cdots, \xi_{n}\right)$ of rational functions on $V$ (the quotient field of $R[V]$ ).

Let $V$ be irreducible and let $W$ be an irreducible algebraic subvariety of $V$. Then $\boldsymbol{p}(V) \subset \boldsymbol{p}(W)$ and $\boldsymbol{p}(W) / \boldsymbol{p}(V)$ is a prime ideal in the ring $\mathbb{R}[V]$. We denote this ideal by $\boldsymbol{p}(W / V)$. If $\left(\eta_{1}, \eta_{2}, \cdots, \eta_{n}\right)$ is the general point of $W$ then we have $\mathbb{R}[V] / \boldsymbol{p}(W / V)=\kappa\left[\eta_{1}, \eta_{2}, \cdots, \eta_{n}\right]$.

We denote by $Q(W / V)$ the quotient ring of $W$ on $V$, that is, the quotient ring of the prime ideal $p(W / V)$ with respect to the ring $\mathbb{R}[V]$. The ring $Q(W / V)$ is a local ring in the sense of Krull [7]. Its maximal ideal shall be denoted by $\boldsymbol{m}(W / V)$; this ideal consists of all nonunits of $Q(W / V)$. The residue field $Q(W / V) / \mathbf{m}(W / V)$ of this local ring coincides with the field $f(W)$.

2. The local vector space $\mathcal{H}(W / V)$.

2.1. The mapping $\mathfrak{m} \rightarrow \mathfrak{m} / \mathfrak{m}^{2}$. We set $\mathfrak{0}=Q(W / V), \mathfrak{m}=\boldsymbol{m}(W / V), \Delta=\mathfrak{o} / \mathfrak{m}$ $=\mathcal{F}(W)$ and we consider the ring $\mathrm{m} / \mathrm{m}^{2}$. This ring, as an additive group, can be regarded as a vector space over $\Delta$ if, for $\bar{u}$ in $\mathfrak{m} / \mathfrak{m}^{2}$ and $\bar{\delta}$ in $\Delta$, we define the product $\bar{\delta} \bar{u}$ as follows:

$$
\bar{\delta} \bar{u}=\mathfrak{m}^{2} \text {-residue of } \delta u,
$$

where $\delta$ is any element of $\boldsymbol{o}$ whose $\mathfrak{m}$-residue is $\bar{\delta}$ and $u$ is any element of $\mathfrak{m}$ whose $\mathfrak{m}^{2}$-residue is $\bar{u}$. It is immediately seen that the product $\bar{\delta} \bar{u}$ is uniquely determined by $\bar{\delta}$ and $\bar{u}$. We call this vector space the local vector space of $V$ at $W$ and we denote this space by $\mathcal{H}(W / V)$. We denote by $\tau$ the mapping $u \rightarrow \bar{u}$ of $\mathfrak{m}$ onto $\mathcal{X}(W / V)$ :

$$
\tau: \quad u \rightarrow \bar{u}, \quad u \in \mathfrak{m}, \quad \bar{u}=\mathfrak{m}^{2} \text {-residue of } u .
$$

Let $v, v_{1}, v_{2}, \cdots, v_{0}$ be elements of $\mathfrak{m}$ and let $\bar{v}=\tau v, \bar{v}_{i}=\tau v_{i}$. If $\delta_{1}, \delta_{2}, \cdots, \delta_{\theta}$ are elements of $\mathfrak{D}$ and if $\bar{\delta}_{i}$ is the $m$-residue of $\delta_{i}$ then the following relalations are equivalent: $\bar{v}=\sum_{i=1}^{v} \bar{\delta}_{i} \bar{v}_{i}, \quad v \equiv \sum_{i=1}^{o} \delta_{i} v_{i}\left(\bmod \mathrm{m}^{2}\right)$. Hence $\bar{v}$ is linearly dependent on $\bar{v}_{1}, \bar{v}_{2}, \cdots, \bar{v}_{\sigma}$ if and only if $v$ belongs to the ideal 
$\mathfrak{D} \cdot\left(v_{1}, v_{2}, \cdots, v_{0}\right)+\mathfrak{m}^{2}$. In particular, if the elements $v_{1}, v_{2}, \cdots, v_{0}$ form $a$ basis for the ideal $\mathrm{m}$, then the vectors $\bar{v}_{1}, \bar{v}_{2}, \cdots, \bar{v}_{o}$ span the entire vector space $\mathcal{H}(W / V)$. Consequently the dimension of this vector space is finite.

Conversely, let us assume that the $g$ vectors $\bar{v}_{i}$ span the entire vector space $\mathscr{H}(W / V)$. We show then that the g elements $v_{i}$ form a basis for the ideal $\mathfrak{m}$. For if we denote by $\mathfrak{A}$ the ideal $\mathfrak{D} \cdot\left(v_{1}, v_{2}, \cdots, v_{0}\right)$, then it follows, by assumption, that $\mathfrak{m}=\mathfrak{A}+\mathfrak{m}^{2}$. Hence $\mathfrak{m}^{2}=\mathfrak{A} \mathfrak{m}+\mathfrak{m}^{3} \subseteq \mathfrak{A}+\mathfrak{m}^{3}$ and therefore $\mathfrak{m}=\mathfrak{A}+\mathfrak{m}^{3}$. This implies $\mathfrak{m}^{2}=\mathfrak{A} \mathfrak{m}+\mathfrak{m}^{4} \subseteq \mathfrak{A}+\mathfrak{m}^{4}$ and therefore $\mathfrak{m}=\mathfrak{A}+\mathfrak{m}^{4}$. In like fashion we find $\mathfrak{m}=\mathfrak{A}+\mathfrak{m}^{i}$ for any integer $i$. Since $\mathfrak{o}$ is a local ring, we have $\bigcap_{\mathfrak{l}=1}^{\infty}\left(\mathfrak{A}+\mathfrak{m}^{i}\right)=\mathfrak{A}([7$, Theorem 2$])$. Hence $\mathfrak{A}=\mathfrak{m}$, and this proves our assertion.

We say that a basis $\left(u_{1}, u_{2}, \cdots, u_{s}\right)$ of $\mathfrak{m}$ is minimal if no proper subset of this basis is a basis of $m$. From the preceding results it follows that $\left(u_{1}, u_{2}, \cdots, u_{8}\right)$ is a minimal basis of $\mathfrak{m}$ if and only if the corresponding vectors $\bar{u}_{1}, \bar{u}_{2}, \cdots, \bar{u}_{s}$ form $a$ (independent) basis of the vector space $\mathcal{X}(W / V)$. All minimal bases of $\mathfrak{m}$ have therefore the same number of elements, this number being equal to the dimension of $\mathscr{N}(W / V)$.

Let $\left(u_{1}, u_{2}, \cdots, u_{s}\right)$ be a minimal basis of $\mathfrak{m}$, so that $s=$ dimension of $\mathcal{H}(W / V)$. We have $u_{i}=f_{i}(\xi) / g_{i}(\xi)$, where $f_{i}(\xi), g_{i}(\xi) \in R[V]$ and $g_{i}(x) \neq 0$ on $W$ (that is, $\left.g_{i}(\xi) \notin p(W / V)\right)$. Since $g_{i}(\xi)$ is a unit in $\mathfrak{o}$, also $f_{1}(\xi)$, $f_{2}(\xi), \cdots, f_{s}(\xi)$ form a minimal basis of $m$. Therefore we may assume without loss of generality that $u_{i} \in R[V], i=1,2, \cdots, s$. Let $r=\operatorname{dimension}$ of $V$ and $\rho=$ dimension of $W$. The finite integral domain $R=R[V]$ has degree of transcendency $r$ over $\kappa$. It is then well known (Krull $[5$, p. 43]) that every isolated prime ideal of the ideal $R \cdot\left(u_{1}, u_{2}, \cdots, u_{s}\right)$ is of dimension not less than $r-s$. Among the isolated prime ideals there is the ideal $p=p(W / V)$, since $\mathfrak{D} \cdot\left(u_{1}, u_{2}, \cdots, u_{s}\right)=\mathfrak{m}=\mathfrak{D} \cdot \mathfrak{p}$, and this prime ideal $\mathfrak{p}$ is of dimension $\rho$. Hence $\rho \geqq r-s$ or $s \geqq r-\rho$, that is, we have the following result: the dimension of the local vector space $\mathfrak{O C}(W / V)$ satisfies the inequality

$$
\operatorname{dim} \mathcal{H}(W / V) \geqq \operatorname{dim} V-\operatorname{dim} W .
$$

In particular, if $W$ is a point $P$ of $V$, then we have:

$$
\operatorname{dim} \mathscr{H}(P / V) \geqq \operatorname{dim} V \text {. }
$$

Two procedures will be used frequently in the sequel for the actual determination of a basis of the vector space $\mathcal{H}(W / V):(1)$ ground field extensions; (2) insertion of varieties between $W$ and $V$. The lemmas given below are intended to introduce these procedures.

2.2. Reduction to dimension zero. Let $\left(\eta_{1}, \eta_{2}, \cdots, \eta_{n}\right)$ be the general point of $W$. If for a given integer $\nu, 1 \leqq \nu \leqq n$, the $\kappa$-homomorphism $\kappa\left[\xi_{1}, \xi_{2}, \cdots, \xi_{n}\right] \sim \kappa\left[\eta_{1}, \eta_{2}, \cdots, \eta_{n}\right]$ which carries $\xi_{i}$ into $\eta_{i}, i=1,2, \cdots, n$, induces an isomorphism between $\kappa\left[\xi_{1}, \xi_{2}, \cdots, \xi_{\nu}\right]$ and $\kappa\left[\eta_{1}, \eta_{2}, \cdots, \eta_{\nu}\right]$, then it is permissible to identify $\xi_{1}, \xi_{2}, \cdots, \xi_{\nu}$ with $\eta_{1}, \eta_{2}, \cdots, \eta_{\nu}$ respec- 
tively. We shall express this assumption and the fact that the identification has actually been performed by writing: $\xi_{i}=\eta_{i}, i=1,2, \cdots, \nu$.

Lemma 1. Let $\xi_{i}=\eta_{i}, i=1,2, \cdots, \nu$, and let $\kappa^{*}$ denote the field $\kappa\left(\xi_{1}, \xi_{2}, \cdots, \xi_{\nu}\right)$. If $V^{*}$ and $W^{*}$ are the varieties (over $\left.\kappa^{*}\right)$ in $S_{n-\nu}^{\kappa^{*}}$ having respectively $\left(\xi_{\nu+1}, \xi_{\nu+2}, \cdots, \xi_{n}\right)$ and $\left(\eta_{v+1}, \eta_{v+2}, \cdots, \eta_{n}\right)$ as general points, then the quotient ring $\mathrm{o}=Q(W / V)$ coincides with the quotient ring $0^{*}=Q\left(W^{*} / V^{*}\right)$ (and hence also the vector spaces $\mathcal{\Re}(W / V), \mathcal{H}\left(W^{*} / V^{*}\right)$ coincide).

Proof. The fact that the identification $\xi_{i}=\eta_{i}, i=1,2, \cdots, \nu$, is permissible, signifies that 0 is the only element common to $\kappa\left[\xi_{1}, \xi_{2}, \cdots, \xi_{\nu}\right]$ and $\mathfrak{p}$ $(=\boldsymbol{p}(W / V))$. Hence the quotient ring $\mathrm{o}$ contains the entire field $\kappa^{*}$. It is clear that $\mathfrak{D} \in \mathfrak{D}^{*}$. On the other hand we have: $R^{*}=R\left[V^{*}\right]=\kappa^{*} R, \mathfrak{p}^{*}=p\left(W^{*} / V^{*}\right)=\kappa^{*} \mathfrak{p}$, and every element of $\kappa^{*} R$ which is not in $\kappa^{*} \mathfrak{p}$ is evidently a unit in $R_{\mathfrak{p}}(=\mathfrak{p})$. Hence $\mathfrak{b}^{*}=R_{\mathfrak{p}^{*}} \subseteq \mathfrak{D}$, and therefore $\mathfrak{D}^{*}=\mathfrak{b}$, as was asserted.

The above lemma includes as a special case the well known reduction to dimension zero. Namely, the dimension of $W$ being $\rho$, we may assume that $\eta_{1}, \eta_{2}, \cdots, \eta_{\rho}$ are algebraically independent over $\kappa$. In that case necessarily also $\xi_{1}, \xi_{2}, \cdots, \xi_{\rho}$ are algebraically independent over $\kappa$, and the identification $\xi_{i}=\eta_{i}, i=1,2, \cdots, \rho$, is permissible. The variety $V^{*}$ of the lemma is now a variety of dimension $n-\rho$ (over $\kappa^{*}=\kappa\left(\xi_{1}, \xi_{2}, \cdots, \xi_{\rho}\right)$ ), immersed in a linear space $S_{n-\rho}^{\kappa^{*}}$, while $W^{*} / \kappa^{*}$ is now a point of $V^{*}$, because $\eta_{\rho+1}, \eta_{\rho+2}$, $\cdots, \eta_{n}$ are algebraic quantities over the new ground field $\kappa^{*}$.

2.3. The linear transformation $\mathcal{X}(W / V) \rightarrow \mathscr{H}\left(W / V^{\prime}\right)$. In the "insertion" procedure we insert an irreducible variety $V^{\prime}$ between $W$ and $V: W \subset V^{\prime} \subset V$, and we consider the two vector spaces $\mathcal{X}=\mathcal{H}(W / V), \mathcal{X}^{\prime}=\mathcal{H}\left(W / V^{\prime}\right)$. These two spaces have the same field of scalars, namely $\mathcal{f}(W)(=\Delta)$. Let $\mathfrak{b}^{\prime}=Q\left(W / V^{\prime}\right), \mathfrak{m}^{\prime}=\boldsymbol{m}\left(W / V^{\prime}\right)$. We have a mapping $\boldsymbol{\tau}^{\prime}$ of $\mathfrak{m}^{\prime}$ onto $\mathcal{X}^{\prime}$ similar to the mapping $\tau$ of $\mathfrak{m}$ onto $\mathcal{X}$ (see (1)):

$$
\tau^{\prime}: \quad u^{\prime} \rightarrow \bar{u}^{\prime}, \quad u^{\prime} \in \mathfrak{m}^{\prime}, \quad \bar{u}^{\prime}=\mathfrak{m}^{\prime 2} \text {-residue of } u^{\prime} .
$$

If $\left(\xi_{1}^{\prime}, \xi_{2}^{\prime}, \cdots, \xi_{n}^{\prime}\right)$ is the general point of $V^{\prime} / \kappa$, there is a definite $\kappa$-homomorphism $\psi$ of $R=R[V]$ onto $R^{\prime}=R\left[V^{\prime}\right]$ which carries $\xi_{i}$ into $\xi_{i}^{\prime}$ (since $\left.V^{\prime} \subset V\right)$, and $\mathfrak{p}=\boldsymbol{p}(W / V)$ is the full inverse image of $\mathfrak{p}^{\prime}=\boldsymbol{p}\left(W / V^{\prime}\right)$ under $\boldsymbol{\psi}^{-1}$. It follows that $\psi$ can be extended (in a unique fashion) to a homomorphism of $\mathrm{D}=R_{\mathfrak{p}}$ onto $\mathfrak{D}^{\prime}=R_{\mathfrak{p}^{\prime}}^{\prime}$. We denote this extended homomorphism by the same letter $\psi$. It is clear that $\psi \mathfrak{m}=\mathfrak{m}^{\prime}$.

We consider in $R[V]$ the ideal $\boldsymbol{p}_{1}=\boldsymbol{p}\left(V^{\prime} / V\right)$. This ideal is the nucleus of the homomorphism $\psi$ of $R$ onto $R^{\prime}$. Since $W \subset V^{\prime}, \mathfrak{p}_{1}$ is contained in $\mathfrak{p}$, and therefore the extended ideal $\mathfrak{B}_{1}=\mathfrak{0} \cdot \mathfrak{p}_{1}$ is a prime ideal contained in $\mathfrak{m}$. The ideal $\mathfrak{P}_{1}$ is the nucleus of the homomorphism $\psi$ of $\mathfrak{D}$ onto $\mathfrak{D}^{\prime}$. If $\mathfrak{A}$ is any $\mathfrak{D}$-ideal contained in $\mathfrak{m}$, then $\tau \mathfrak{A}$ is a linear subspace of $\mathcal{H}$. We consider in particular the subspace $\tau \mathfrak{B}_{1}$ of $\mathcal{X}$. This subspace is spanned by the vectors belonging to the subset $\tau p_{1}$ of $\mathcal{X}$. 
Lemma 2. The transformation $\phi=\tau^{-1} \psi \tau^{\prime}$ is single-valued and is a linear transformation of $\mathcal{X}=\mathscr{H}(W / V)$ onto $\mathcal{X}^{\prime}=\mathcal{H}\left(W / V^{\prime}\right)$. The subspace of $\mathcal{X}$ which is annihilated by $\phi$ is the space spanned by the vectors belonging to $\tau p_{1}$ (where $p_{1}$ is the ideal $\boldsymbol{p}\left(V^{\prime} / V\right)$ of $V^{\prime}$ in $\left.\mathbb{R}[V]\right)$, or also the space $\tau \mathfrak{B}_{1}\left(\right.$ where $\left.\mathfrak{B}_{1}=\mathfrak{0} \cdot \mathfrak{p}_{1}\right)$.

Proof. Since $\tau$ and $\tau^{\prime}$ are respectively transformations of $\mathfrak{m}$ onto $\mathcal{X}$ and of $\mathfrak{m}^{\prime}$ onto $\mathcal{H}^{\prime}$, and since $\psi \mathfrak{m}=\mathfrak{m}^{\prime}, \psi$ is a transformation of $\mathcal{X}$ onto $\mathfrak{H}^{\prime}$. If $\bar{u}$ is any element of $\mathcal{X}$, then $\tau^{-1} \bar{u}$ is a residue class $u+\mathfrak{m}^{2}$ in $\mathfrak{m}$ modulo $\mathfrak{m}^{2}$; $\psi\left(u+\mathfrak{m}^{2}\right)=\psi u+\psi\left(\mathfrak{m}^{2}\right)=u^{\prime}+\mathfrak{m}^{\prime 2}$, since $\psi$ is a homomorphism and since $\psi \mathfrak{m}=\mathfrak{m}^{\prime}$; and finally $\tau^{\prime}\left(u^{\prime}+\mathfrak{m}^{\prime 2}\right)=\tau^{\prime} u^{\prime}=\bar{u}^{\prime}$. Hence $\psi$ is single-valued. Since $\tau$, $\psi$ and $\tau^{\prime}$ are homomorphisms of additive groups, it follows that $\phi$ is a homomorphism of the additive group $\mathcal{X}$ onto the additive group $\mathcal{X}^{\prime}$. If $\bar{\delta} \in \Delta(=\mathcal{F}(W))$ and $\bar{u} \in \mathcal{X}$, let $u$ be some element in $\tau^{-1} \bar{u}$ and let $\delta$ be some element in $\mathfrak{D}$ whose $\mathfrak{m}$-residue is $\bar{\delta}$. We have, by the definition of $\mathcal{X}$ and $\tau:(\tau \phi) \delta u=\phi(\bar{\delta} \bar{u})$. On the other hand, $\bar{\delta}$ is also the $\mathfrak{m}^{\prime}$-residue of $\psi(\delta)$, and therefore $\left(\psi \tau^{\prime}\right) \delta u$ $=\tau^{\prime}(\psi(\delta) \psi(u))=\bar{\delta} \cdot\left(\psi \tau^{\prime}\right) u$. Since $\tau \phi=\psi \tau^{\prime}$ we conclude that $\phi(\bar{\delta} \bar{u})=\bar{\delta} \cdot \phi \bar{u}$ which shows that $\phi$ is linear. Finally, we have $(\tau \phi) u=\phi(\bar{u})$ and $(\tau \phi) u=\left(\psi \tau^{\prime}\right) u$. Hence $\phi(\bar{u})=0$ if and only if $\left(\psi \tau^{\prime}\right) u=0$, that is, if and only if $\psi u \in \mathfrak{m}^{\prime 2}$. Since the nucleus of the homomorphism $\psi$ of $\mathfrak{D}$ onto $\mathfrak{D}^{\prime}$ is the ideal $\mathfrak{P}_{1}$ defined above, we conclude that $\phi(\bar{u})=0$ if and only if $u \in \mathfrak{m}^{2}+\mathfrak{B}_{1}$, and this shows that the subspace of $\mathcal{X}$ annihilated by $\phi$ is the space $\tau \mathfrak{B}_{1}$. This completes the proof of the lemma.

CoRollary. If $u_{1}, u_{2}, \cdots, u_{\nu}$ are elements of $m$ such that $\psi u_{1}, \psi u_{2}, \cdots, \psi u_{\nu}$ form a basis of $\mathfrak{m}^{\prime}$, and if $u_{\nu+1}, u_{\nu+2}, \cdots, u_{\nu+\mu}$ form a basis of $\mathfrak{B}_{1}$, then the $\nu+\mu$ elements $u_{i}$ form a basis of $\mathrm{m}$.

\section{Simple points.}

3.1. Definition of simple loci. The inequality $\left(2^{\prime}\right)$ assigns a lower limit to the dimension of the local vector space at any point $P$ of a given variety $V$. This lower limit is the dimension $r$ of $V$. More generally, by (2), the difference $r-\rho$ is a lower limit for the dimension of the local vector space at any $\rho$-dimentional irreducible subvariety $W$ of $V$. We speak of simple points and of simple subvarieties of $V$ when these lower limits are reached, that is, we give the following definition:

Definition 1. A point $P$ of $V$ is simple if $\operatorname{dim} \mathcal{X}(P / V)=r=\operatorname{dim} V$. More generally, a $\rho$-dimensional irreducible subvariety $W$ of $V$ is simple if $\operatorname{dim} \mathcal{H}(W / V)$ $=r-\rho$.

According to this definition, a $\rho$-dimensional irreducible subvariety $W$ of $V$ is simple if the maximal ideal $\boldsymbol{m}(W / V)$ of the quotient ring $Q(W / V)$ has a basis of $r-\rho$ elements (such a basis is then necessarily minimal). If $W$ is simple, the $r-\rho$ elements of any minimal basis of $\boldsymbol{m}(W / V)$ shall be called uniformizing parameters of $W$.

$W$ is singular for $V$ if it is not simple.

We observe that our definition of simple loci has an intrinsic character, 
since the ambient linear space $S_{n}$ does not intervene at all in the definition. Whether a given irreducible subvariety $W$ of $V$ is or is not simple for $V$ depends entirely on the structure of the quotient ring $Q(W / V)$. This puts in evidence the invariantive character of the concept of simple loci with respect to regular transformations: if $W$ is simple for $V$ and if $V$ is transformed into another variety $V^{*}$ by a birational transformation which is regular at $W$ (see $[13$, p. 513$]$ ), then to $W$ there corresponds on $V^{*}$ a (unique) subvariety $W^{*}$ which is also simple for $V^{*}$ (and has the same quotient ring as $W$ ).

3.2. Geometric aspects of the definition. In this section we shall discuss some geometric aspects of our definition of a simple point. For that purpose we shall examine the variety $V$, together with a given point $P$ on it, in relation to the ambient linear space $S_{n}$ in which $V$ is immersed.

We have shown elsewhere that every point $P$ of $S_{n}$ is simple (see [13], Lemma 9, p. 541 and first footnote on that page). It is easy to detect in our proof of this result a specialization of the general set-up dealt with in Lemma 2. Namely, we apply that lemma to the following case: $V=S_{n}, W=P$ $=P\left(\alpha_{1}, \alpha_{2}, \cdots, \alpha_{n}\right)$ and $V^{\prime}=$ the variety having $\left(\alpha_{1}, x_{2}, x_{3}, \cdots, x_{n}\right)$ as general point. The ideal $p\left(V^{\prime} / V\right)$ is in this case the principal ideal $\left(f\left(x_{1}\right)\right)$ in $\kappa\left[x_{1}, x_{2}, \cdots, x_{n}\right]$, where $f\left(x_{1}\right)$ is an irreducible polynomial in $\kappa\left[x_{1}\right]$ such that $f\left(\alpha_{1}\right)=0$. Hence the space annihilated by the linear transformation $\phi$ is at most of dimension 1 , and therefore $\operatorname{dim} \mathscr{x}\left(P / S_{n}\right) \leqq 1+\operatorname{dim} \mathcal{X}\left(P / V^{\prime}\right)$. If we now replace in Lemma 1 the varieties $V$ and $W$ by $V^{\prime}$ and $P$ respectively, the lemma is applicable if we set $\nu=1$, since in the present case we have $\xi_{1}=\eta_{1}=\alpha_{1}$. Therefore we conclude that $\mathscr{X}\left(P / V^{\prime}\right)=\mathscr{H}\left(P^{*} / S_{n-1}^{\kappa^{*}}\right)$, where $\kappa^{*}=\kappa\left(\alpha_{1}\right)$ and $P^{*}$ is the point $\left(\alpha_{2}, \alpha_{3}, \cdots, \alpha_{n}\right)$ over $\kappa^{*}$. We have therefore: $\operatorname{dim} \mathfrak{H}\left(P / S_{n}\right)$ $\leqq 1+\operatorname{dim} \mathcal{X}\left(P^{*} / S_{n-1}^{*^{*}}\right)$. Applying the same argument to the point $P^{*}$, and repeating this procedure $n$ times, we shall get ultimately the inequality: $\operatorname{dim} \mathcal{H}\left(P / S_{n}\right) \leqq n$. Hence necessarily $\operatorname{dim} \mathcal{H}\left(P / S_{n}\right)=n$, and therefore $P$ is a simple point of $S_{n}$.

We shall now proceed by analogy with the classical case, where $\kappa$ is the field of complex numbers, or, more generally, an algebraically closed field. In that case the coordinates $\alpha_{1}, \alpha_{2}, \cdots ; \alpha_{n}$ of any point $P$ in $S_{n}$ are in $\kappa$, and the $n$ differences $x_{i}-\alpha_{i}$ form a minimal base of $m\left(P / S_{n}\right)$, that is, they are uniformizing parameters of $P$. The field of scalars $\Delta$ of the vector space $\mathcal{X}\left(P / S_{n}\right)$ is the field $\kappa$ itself, since $\Delta=\kappa\left(\alpha_{1}, \alpha_{2}, \cdots, \alpha_{n}\right)$. Hence, this vector space can be identified with the space of all linear forms in $x_{1}-\alpha_{1}, x_{2}-\alpha_{2}, \cdots, x_{n}-\alpha_{n}$ with coefficients in $\kappa$. If $u=\sum_{i=1}^{n} c_{i}\left(x_{i}-\alpha_{i}\right)+$ terms of degree greater than 1 in the $x_{i}-\alpha_{i}$ is any polynomial in $\kappa\left[x_{1}, x_{2}, \cdots, x_{n}\right]$ which vanishes at $P$, then under the mapping $\tau$ of $\boldsymbol{m}\left(P / S_{n}\right)$ onto $\mathscr{H}\left(P / S_{n}\right)$ we get $\tau u=\sum_{i=1}^{n} c_{i}\left(x_{i}-\alpha_{i}\right)$. The classroom definition of a simple point of a hypersurface $H$, given by an equation $u=0$, takes therefore the following form: a point $P$ of the hypersurface $H$ is simple if and only if the vector $\tau u$ in $\mathscr{x}\left(P / S_{n}\right)$ is different from zero: 
At a simple point $P$ the vector $\tau u$ fixes the position of the tangent hyperplane at $P$ of the hypersurface $H$.

The above italicized statement can be of course easily proved in the abstract case on the basis of our definition of simple points. However, since we have defined simple points only for irreducible varieties, we must suppose that we are dealing with an irreducible hypersurface. We apply Lemma 2 to the case $V=S_{n}, V^{\prime}=H, W=P$. Since the ideal $p\left(V^{\prime} / V\right)$ is now the principal ideal $(u)$, the subspace of $\mathscr{H}\left(P / S_{n}\right)$ which is annihilated by $\phi$ is spanned by the single vector $\tau u$. Consequently the dimension of the vector space $\mathcal{X}(P / H)$ is $n$ or $n-1$, according as $\tau u=0$ or $\tau u \neq 0$. Since $H$ is of dimension $r=n-1$, the statement follows.

In the case of a reducible hypersurface $H$ we shall define simple points just by the above criterion $\tau u \neq 0$. Let $u=\prod_{i=1}^{p} u_{i}^{\nu_{i}}$ be the decomposition of $u$ into irreducible factors, and let $H_{i}$ denote the hypersurface $u_{i}=0$. The condition $\tau u \neq 0$ signifies that $u \in \mathfrak{m}, u \in \mathfrak{m}^{2}$, where $\mathfrak{m}=\boldsymbol{m}\left(P / S_{n}\right)$. That is equivalent to the following set of conditions: (1) one, and only one, of the polynomials $u_{1}, u_{2}, \cdots, u_{g}$ is in $\mathfrak{m}$; (2) if, say, $u_{1} \in \mathfrak{m}$, then $u_{1} \in \mathfrak{m}^{2}$ and $\nu_{1}=1$. Or in geometric language: $P$ is a simple point of $H$ if and only if the following conditions are satisfied: (1) only one of the irreducible components $H_{i}$ of the hypersurface $H$ goes through $P$; (2) if, say, $H_{1}$ contains $P$, then $P$ is a simple point of $H_{1}$ and $H_{1}$ is a simple component of $H$.

If $P$ is a simple point of our hypersurface $H$, we define as tangent hyperplane of $H$ at $P$ the one-dimensional subspace of $\mathcal{H}\left(P / S_{n}\right)$ spanned by the vector $\tau u$. A true picture of the set of all hyperplanes through $P$ is the bundle of hyperplanes in an affine $n$-dimensional space over $\Delta \quad(=\mathfrak{F}(P)$ $\left.=\kappa\left(\alpha_{1}, \alpha_{2}, \cdots, \alpha_{n}\right)\right)$ with center at the origin.

Given $\nu$ hypersurfaces $H_{i}: u_{i}=0, i=1,2, \cdots, \nu$, passing simply through $P$, we say that their tangent hyperplanes are linearly independent if the vectors $\tau u_{1}, \tau u_{2}, \cdots, \tau u_{\nu}$ in $\mathscr{H}\left(P / S_{n}\right)$ are linearly independent. The following result is well known [3, corollary on p. 87]:

Lemma 3. If $P$ is a simple point of an irreducible $r$-dimensional algebraic variety $V$ and if $u_{1}, u_{2}, \cdots, u_{\nu}$ are elements of $\boldsymbol{m}(P / V)$ such that the corresponding vectors $\tau u_{1}, \tau u_{2}, \cdots, \tau u_{\nu}$ of $\mathcal{X}(P / V)$ are linearly independent, then the ideal $p=\left(u_{1}, u_{2}, \cdots, u_{v}\right)$ in $Q(P / V)$ is prime and $(r-\nu)$-dimensional.

Corollary. The assumptions being as in Lemma 3 , the irreducible $(r-\nu)$ dimensional subvariety $W$ of $V$ passing through $P$ which is defined by the ideal $p$ is simple for $V$.

For if $\mathfrak{p}=Q(P / V)$, then $Q(W / V)=\mathfrak{o}_{\mathfrak{p}}$ and $\boldsymbol{m}(W / V)=\mathfrak{o}_{\mathfrak{p}} \cdot \mathfrak{p}=\mathfrak{o}_{\mathfrak{p}} \cdot\left(u_{1}, u_{2}\right.$, $\left.\cdots, u_{\nu}\right)$. Hence $\operatorname{dim} \mathcal{H}(W / V) \leqq \nu=r-\operatorname{dim} W$, and therefore, by (2), $\operatorname{dim}$ $\mathcal{X}(W / V)=\nu$.

For $V=S_{n}$ it follows from this lemma that if $\nu$ hypersurfaces $H_{i}$ pass 
simply through a point $P$ and have at $P$ linearly independent tangent hyperplanes, then locally, at $P$, the complete intersection $W$ of the hypersurfaces $H_{i}$ is irreducible and of dimension $n-\nu$. We shall say then that $W$ is locally, at $P$, regular intersection of the hypersurfaces $H_{i}$ (the "regularity" assumption signifies not only that $W$ is complete intersection of $H_{i}$ locally at $P$, but also that the tangent hyperplanes of the hypersurfaces $H_{i}$ at $P$ are linearly independent).

After these preliminaries, we can express the geometric content of our definition of simple points by means of the following result:

THEOREM 1. A point $P$ of an $r$-dimensional algebraic irreducible variety $V$ in $S_{n}$ is simple for $V$, if and only if $V$ is locally at $P$ a regular intersection of $n-r$ hypersurfaces.

Proof. If in Lemma 2 we identify $W, V^{\prime}$ and $V$ with $P, V$ and $S_{n}$ respectively and if we take into account the inequality $\left(2^{\prime}\right)(\$ 2.1)$, we conclude (independently of the assumption that $P$ is simple for $V$ ) that the ideal $p(V)$ in $\kappa\left[x_{1}, x_{2}, \cdots, x_{n}\right]$ cannot contain more than $n-r$ polynomials $u_{i}$ such that the corresponding vectors $\tau u_{i}$ in $\mathcal{H}\left(P / S_{n}\right)$ are linearly independent. The point $P$ is simple for $V$ if and only if the maximum $n-r$ is reached. Hence $P$ is simple for $V$ if and only if there exist $n-r$ hypersurfaces $H_{i}$ containing $V$ and having at $P$ linearly independent tangent hyperplanes. The complete intersection of the $H_{i}$, locally at $P$, being of dimension $r$ and containing $V$, it must coincide with $V$, q.e.d.

3.3. Local ideal bases at a simple point. In the preceding discussion the linear space $S_{n}$ played the privileged role of a universal ambient space. We now replace the $S_{n}$ by a fixed $n$-dimensional irreducible variety $S$ (immersed in some linear space of a higher dimension). We consider a fixed simple point $\boldsymbol{P}$ of $\boldsymbol{S}$ and we proceed to develop some aspects of the local icieal theory of $\boldsymbol{S}$ at $P$ similar to those developed above for the $S_{n}$.

We denote by $R$ the coordinate ring $R[S]$ and by $\mathfrak{D}$ the quotient ring $Q(P / S)$. Given any ideal $\mathfrak{A}$ in $R$, we say that a set of elements $\omega_{1}, \omega_{2}, \cdots, \omega_{8}$ in $R$ is a local $P$-basis of $\mathfrak{A}$ if this set is a basis for the ideal $\mathfrak{D} \cdot \mathfrak{A}$; and that it is a minimal local $P$-basis if no proper subset of the set $\left(\omega_{1}, \omega_{2}, \cdots, \omega_{s}\right)$ is a local $P$-basis.

We observe that if $\mathfrak{B}$ is any ideal in $\mathfrak{D}$ and if $\mathfrak{m}$ is the maximal ideal of $\mathfrak{D}$, then the residue class ring $\mathfrak{B} / \mathfrak{m} \mathfrak{B}$, as an additive group, can be regarded as a vector space $\mathcal{N}$ over the field $\Delta=\mathrm{D} / \mathfrak{m}=$ residue field of $P$. This is true in any local ring $D$, and the definition of that vector space is the same as the one given in $\$ 2.1$ for the special case $\mathfrak{B}=\mathfrak{m}$.

By exactly the same reasoning which was employed in that special case it can be proved that $\left(u_{1}, u_{2}, \cdots, u_{v}\right)$ is a basis of $\mathfrak{B}$ if and only if the corresponding vectors $\bar{u}_{1}, \bar{u}_{2}, \cdots, \bar{u}_{\nu}$ span the entire space $\mathcal{N}$, and that $\left(u_{1}, u_{2}, \cdots, u_{v}\right)$ is a minimal basis of $\mathfrak{B}$ if and only if the vectors $\bar{u}_{1}, \bar{u}_{2}, \cdots, \bar{u}_{\nu}$ 
form an independent basis of $\mathcal{N}$. Hence all minimal bases of $\mathfrak{B}$ have the same number of elements.

In particular, we conclude that all minimal local $P$-bases of a given ideal $\mathfrak{A}$ in $R$ have the same number of elements.

We now extend and further elaborate Theorem 1 as follows:

THEOREM 2. If $V$ is an irreducible algebraic subvariety of $\mathbf{S}$ passing through $P$, then $P$ is simple for $V$ if and only if either one of the following two conditions is satisfied:

(1) The dimension of $V$ being $r$, the ideal $p(V / S)$ contains $n-r$ elements $u_{1}, u_{2}, \cdots, u_{n-r}$ such that the corresponding vectors $\tau u_{1}, \tau u_{2}, \cdots, \tau u_{n-r}$ in $\mathcal{H}(P / S)$ are linearly independent.

(2) The ideal $p(V / S)$ possesses a local $P$-basis $u_{1}, u_{2}, \cdots, u_{s}$ such that the vectors $\tau u_{1}, \tau u_{2}, \cdots, \tau u_{s}$ are linearly independent.

Moreover, if condition (1) is satisfied, then the $n-r$ elements $u_{i}$ form necessarily a minimal local $P$-basis of $p(V / S)$, and any minimal local $P$-basis of $\boldsymbol{p}(V / S)$ necessarily consists of elements $v_{1}, v_{2}, \cdots, v_{n-r}$ of $R$ such that the vectors $\tau v_{1}, \tau v_{2}, \cdots, \tau v_{n-r}$ are independent. If condition (2) holds then $\operatorname{dim} V=n-s$ and $\left(u_{1}, u_{2}, \cdots, u_{s}\right)$ is necessarily a.minimal local $P$-basis of $p(V / S)$.

Proof. We have $P \subseteq V \subseteq S$ and $\operatorname{dim} \mathcal{H}(P / S)=n$. Therefore, by Lemma 2, $\operatorname{dim} \mathcal{H}(P / V)=\operatorname{dim} V$ if and only if $\boldsymbol{p}(V / S)$ contains $n-r$ elements $u_{i}$ such as specified in condition (1) (no more than $n-r$ such elements can belong to $\boldsymbol{p}(V / S)$ because $\operatorname{dim} \mathcal{H}(P / V)$ is always greater than or equal to $\operatorname{dim} V)$. Hence condition (1) is both necessary and sufficient.

By Lemma 3, the $n-r$ elements of condition (1) generate in $D$ a prime ideal of dimension $r$, and this ideal is contained in the ideal $\mathfrak{D} \cdot \mathfrak{A}$, where $\mathfrak{A}=\boldsymbol{p}(V / S)$. Since also $\mathfrak{o} \cdot \mathfrak{A}$ is prime and of dimension $r$, it follows that $\left(u_{1}, u_{2}, \cdots, u_{n-r}\right)$ is a local $P$-basis of $\boldsymbol{p}(V / S)$. This shows that condition (2) is necessary. On the other hand, if this condition is satisfied, then again, by Lemma 3 , we have necessarily $\operatorname{dim} V=n-s$, and by Lemma 2 we find $\operatorname{dim} \mathfrak{X}(P / V)=n-s$. Therefore condition (2) is also sufficient.

No subset of set $\left(u_{1}, u_{2}, \cdots, u_{n-r}\right)$ of condition (1) can be a local $P$-basis of $\boldsymbol{p}(V / \mathbf{S})$ for otherwise $V$ would be of dimension greater than $r$.

To complete the proof of the theorem we proceed as follows. Assuming that condition (1) is satisfied, let $\mathfrak{B}=\mathfrak{0} \cdot \boldsymbol{p}(V / \boldsymbol{S})$. We have $\mathfrak{B m} \subseteq \mathfrak{m}^{2} \cap \mathfrak{B}$. On the other hand, if $\omega$ is any element of $\mathfrak{m}^{2} \cap \mathfrak{B}$, then $\omega=\delta_{1} u_{1}+\delta_{2} u_{2}+\ldots$ $+\delta_{n-r} u_{n-r}$, where $\delta_{i} \in \mathfrak{0}$. Since $\omega \in \mathfrak{m}^{2}$ we have $\bar{\delta}_{1} \bar{u}_{1}+\bar{\delta}_{2} \bar{u}_{2}+\cdots+\bar{\delta}_{n-r} \bar{u}_{n-r}=0$, where $\bar{\delta}_{i}=\mathrm{m}$-residue of $\delta_{i}$ and $\bar{u}_{i}=\tau u_{i}$. Since the vectors $\tau u_{i}$ are linearly independent, it follows that $\bar{\delta}_{i}=0$, that is, $\delta_{i} \in \mathfrak{m}, i=1,2, \cdots, n-r$. Hence $\omega \in \mathfrak{B m}$. We have thus proved that $\mathfrak{B m}=\mathfrak{m}^{2} \cap \mathfrak{B}$. But this implies that the vector space $\mathfrak{B} / \mathscr{B} \mathfrak{m}$ can be regarded as a subspace of the vector space $\mathfrak{m} / \mathfrak{m}^{2}=(\mathscr{H}(P / S))$. Since we know that the elements of a minimal basis of $\mathscr{B}$ give rise to independent vectors of $\mathfrak{B} / \mathfrak{B m}$, we conclude that the elements of such a basis must 
also give rise to independent vectors of $\mathscr{H C}(P / S)$. This completes the proof of the theorem.

3.4. Simple zeros of ideals. The criterion for simple points given by condition (2) of Theorem 2 expresses a property of the ideal $\boldsymbol{p}(V / \boldsymbol{S})$ which continues to have a meaning if instead of $\boldsymbol{p}(V / S)$ we are dealing with an arbitrary ideal $\mathfrak{A}$ in $R(=\mathbb{R}[S])$. This leads to a definition of a simple zero of an ideal $\mathfrak{A}$, and in particular of a simple point of a reducible algebraic variety:

DEFINITION 2. Given an arbitrary ideal $\mathfrak{A}$ in $\mathbb{R}[\mathbf{S}]$ and.given a simple point $P(\alpha)$ of $S$, we say that $(\alpha)$ is a simple zero of $\mathfrak{U}$ if $(\alpha)$ is a zero of $\mathfrak{A}$ and if $\mathfrak{A}$ possesses a local $P$-basis $\left(u_{1}, u_{2}, \cdots, u_{s}\right)$ such that the vectors $\tau u_{1}, \tau u_{2}, \cdots, \tau u_{s}$ in $\mathcal{H}(P / S)$ are independent. In particular, given an arbitrary subvariety $V$ of $S$ (not necessarily irreducible), we say that $P$ is simple for $V$ if $(\alpha)$ is a simple zero of $I(V / \mathbf{S})$.

Concerning this definition the following observations should be made. By Lemma 3 , the elements $u_{1}, u_{2}, \cdots, u_{s}$ generate in $\mathfrak{D}$ a prime ideal $\mathfrak{B}$ of dimension $n-s$. If $\mathfrak{p}=\mathfrak{P} \cap \mathbb{R}[S]$ then we have $\mathfrak{D} \cdot \mathfrak{A}=\mathfrak{P}=\mathfrak{D} \cdot \mathfrak{p}$. Moreover, since $\left(u_{1}, u_{2}, \cdots, u_{s}\right)$ is also a local basis of $\mathfrak{p}$, it follows from Theorem 2 that $P$ is a simple point of the $(n-s)$-dimensional irreducible variety $U(\mathfrak{p})$. We thus see that the condition that $(\alpha)$ be a simple zero of $\mathfrak{A}$ signifies that locally, at $P$, the ideal $\mathfrak{A}$ does not differ essentially from a prime ideal $\mathfrak{p}$ (that is, we have $\mathfrak{o} \cdot \mathfrak{A}=\mathfrak{o} \cdot \mathfrak{p})$ and that the variety $\mathcal{U}(\mathfrak{p})$ of this prime ideal has a simple point at $P$. We also point out that, in terms of a normal decomposition of $\mathfrak{A}$ into primary components, the relation $\mathfrak{D} \cdot \mathfrak{A}=\mathfrak{D} \cdot \mathfrak{p}$ expresses the fact that $\mathfrak{p}$ itself is one of the primary (necessarily isolated) components of $\mathfrak{A}$ and that $\mathfrak{p}$ is the only prime ideal of $\mathfrak{A}$ which is contained in the prime ideal $p(P)$ of the point $P$.

We must point out explicitly that in the second part of our definition we speak of an algebraic variety $V$ in a strictly set-theoretic sense. There is no question of multiple components or of embedded components; $V$ is simply a set of points. This is clearly indicated by the fact that our definition is in terms of the ideal $I(V)$, this ideal being its own radical and therefore a finite intersection of prime ideals. The observations just made on the simple zeros of an arbitrary ideal show that, according to our definition, a point $P$ of an algebraic variety $V$ is simple if and only if $V$ has only one irreducible component through $P$ and this component has a simple point at $P$.

For simple zeros of an ideal $\mathfrak{A}$ one could formulate results similar to those stated in Theorem 2. For later applications we shall state explicitly the following theorem:

THEOREM $2^{\prime}$. If $(\alpha)$ is a zero of an $r$-dimensional isolated prime ideal $\mathfrak{p}$ of $\mathfrak{A}$ (that is, if the point $P(\alpha)$ belongs to an $r$-dimensional irreducible component of the variety $\mathcal{U}(\mathfrak{A}))$ and if $\left(\omega_{1}, \omega_{2}, \cdots, \omega_{\nu}\right)$ is a local $P$-basis of $\mathfrak{A}$, then $(\alpha)$ is a simple zero of $\mathfrak{A}$ if and only if exactly $n-r$ of the vectors $\tau \omega_{1}, \tau \omega_{2}, \cdots, \tau \omega_{\nu}$ in $\mathcal{O C}(P / S)$ are linearly independent. 
Proof. From the preceding considerations we know that in order that $(\alpha)$ be a simple zero of the ideal $\mathfrak{A}$ it is necessary and sufficient that two conditions be satisfied:

(a) We must have $\mathfrak{D} \cdot \mathfrak{A}=\mathfrak{D} \cdot \mathfrak{p}$. This condition is equivalent to the following: $\left(\omega_{1}, \omega_{2}, \cdots, \omega_{\nu}\right)$ is a local $P$-basis of $\mathfrak{p}$.

(b) $P$ must be a simple point of the variety $U(\mathfrak{p})$.

If $(\alpha)$ is a simple zero of $\mathfrak{A}$, then by (a) the set $\left(\omega_{1}, \omega_{2}, \cdots, \omega_{\nu}\right)$ contains a minimal local $P$-basis of $\mathfrak{p}$. Let $\left(\omega_{1}, \omega_{2}, \cdots, \omega_{\sigma}\right)$ be such a basis. By (b) and by the second part of Theorem 2, we must have $\sigma=n-r$, and the vectors $\tau \omega_{1}, \tau \omega_{2}, \cdots, \tau \omega_{n-r}$ must be linearly independent. By Lemma 3 no more than $n-r$ of the $\nu$ vectors $\tau \omega_{i}$ can be independent, since $\operatorname{dim} \mathfrak{p}=r$.

Conversely, if $n-r$ of the vectors $\tau \omega_{i}$, say $\tau \omega_{1}, \tau \omega_{2}, \cdots, \tau \omega_{n-r}$, are independent, then by condition (1) of Theorem 2, $P$ is a simple point of $U(p)$. By Lemma 3 , the $n-r$ elements $\omega_{1}, \omega_{2}, \cdots, \omega_{n-r}$ form then a local $P$-basis of $\mathfrak{p}$. Hence, a fortiori the $\nu$ elements $\omega_{1}, \omega_{2}, \cdots, \omega_{\nu}$ form a local $P$-basis of $\mathfrak{p}$. Therefore conditions (a) and (b) are satisfied, and $(\alpha)$ is a simple zero of $\mathfrak{A}$.

\section{Simple subvarieties.}

4.1. Generalization of the preceding results. If $W$ is a $\rho$-dimensional irreducible variety in $S_{n}$, the reduction to the zero-dimensional case (\$2.2) has the effect of replacing $S_{n}$ by an $S_{n-\rho}^{\kappa^{*}}$ and $W$ by a point $P^{*}\left(=W^{*}\right)$ in this $S_{n-\rho}^{x^{*}}$. Every irreducible $r$-dimensional variety $V$ in $S_{n}$ containing $W$ is then replaced by an $(r-\rho)$-dimensional irreducible variety $V^{*} / \kappa^{*}$ in $S_{n-\rho}^{\kappa^{*}}$ containing $P^{*}$. Since this reduction has no effect on the quotient ring $Q(W / V)$ (Lemma 1 ), the results of the preceding section are either valid or can be properly interpreted if we deal with $W$ instead of with a point $P$ in $S_{n}$. We shall briefly state the corresponding results using $W$ instead of $P$.

Every variety $W$ in $S_{n}$ is a simple subvariety of $S_{n}$, that is, $\operatorname{dim} \mathcal{H}\left(W / S_{n}\right)$ $=n-\rho$. A set of uniformizing parameters of $W$ in $S_{n}$ consists of $n-\rho$ elements. If $W$ is a simple subvariety of an $n$-dimensional irreducible variety $S$ and if $u_{1}, u_{2}, \cdots, u_{\nu}$ are elements of $\boldsymbol{m}(W / S)$ such that the corresponding vectors $\tau u_{1}, \tau u_{2}, \cdots, \tau u_{\nu}$ in $\mathcal{X}(W / S)$ are independent, then the ideal $\left(u_{1}, u_{2}, \cdots, u_{\nu}\right)$ in $Q(W / S)$ is prime and of dimension $n-\nu$ (see Lemma 3). Here the dimension is intended with respect to the given ground field $\kappa$. After the reduction to the zero-dimensional case the dimension $n-\nu$ of Lemma 3 becomes $(n-\rho)-\nu$, but this is the dimension of the ideal $\left(u_{1}, u_{2}, \cdots, u_{v}\right)$ in $Q(W / S)$ with respect to a field $\kappa^{*}$ which is of degree of transcendency $\rho$ over $\kappa$ (see $\$ 2.2$, Lemma 1). If $\mathfrak{D}=Q(W / S)$ and if $\mathfrak{A}$ is any ideal in $R[S]$, then any set of elements of $R[S]$ which is a basis of $\mathfrak{D} \cdot \mathfrak{A}$ is called a local $W$-basis of $\mathfrak{A}$. All minimal local $W$-bases of a given ideal $\mathfrak{A}$ in $R[S]$ have the same number of elements. Theorem 2 remains valid if $P$ is replaced by $W$ and $\mathcal{X}(P / S)$ by $\mathscr{X}(W / \mathbf{S})$. Thus $W$ is simple for $V$ ( $V$ irreducible) if and only if either one of the following conditions is satisfied: (1) $\operatorname{dim} V=r$ and the ideal $\boldsymbol{p}(V / S)$ contains $n-r$ elements $u_{i}$ such that the vectors $\tau u_{1}, \tau u_{2}, \cdots, \tau \dot{u}_{n-r}$ in $\mathcal{X}(W / S)$ 
are independent; $(2)$ the ideal $p(V / S)$ possesses a local $W$-basis $\left(u_{1}, u_{2}, \cdots, u_{s}\right)$ such that the $s$ vectors $\tau u_{i}$ are independent. (Note that by the reduction to the zero-dimensional case the difference $n-r$ remains invariant: $n-r$ $=(n-\rho)-(r-\rho) ;$ moreover we have $\operatorname{dim} V^{*} / \kappa^{*}=(n-\rho)-s=(n-s)-\rho$, whence $\operatorname{dim} V / \kappa=n-s$.) If $(\eta)$ is the general point of $W$, then Definition 2 reads as follows: $(\eta)$ is a simple zero of an ideal $\mathfrak{A}$ in $R=\mathbb{R}[S]$ if $\mathfrak{A}$ possesses a local $W$-basis $\left(u_{1}, u_{2}, \cdots, u_{s}\right)$ such that $\tau u_{1}, \tau u_{2}, \cdots, \tau u_{s}$ are independent vectors (the reduction to dimension zero leads to elements $u_{1}{ }^{*}, u_{2}{ }^{*}, \cdots, u_{s}^{*}$ in $\kappa^{*} R$, but since $\kappa^{*} \subseteq Q(W / S)$ these $u_{i}^{*}$ can be replaced by elements in $\left.R\right)$. In particular, if $V$ is any algebraic variety (not necessarily irreducible) and if $W \subseteq V$ then $W$ is simple for $V$ if and only if $W$ belongs to only one irreducible component of $V$ and $W$ is simple for that component.

4.2. Singular subvarieties and singular points. The customary geometric way of looking at singular subvarieties of dimension greater than 0 is given by the following statement, which we shall formulate as a theorem:

Theorem 3. An irreducible subvariety $W$ of a variety $V$ is singular for $V$ if and only if all the points of $W$ are singular for $V$.

This theorem follows as an immediate consequence from an algebraic criterion for simple loci which will be proved later on (see \$9.6) and which makes use of derivatives and of certain mixed Jacobian matrices. However, it is of interest from a methodological point of view to prove Theorem 3 without making use of differentiation arguments. Such a proof, based directly on our definition of simple loci, must be essentially a local argument and as such is likely to contain elements of interest for the general theory of local or semi-local rings.

Before we proceed with the proof of Theorem 3, we point out the following corollary of this theorem:

COROLlaRY. Every irreducible variety $V$ carries simple points.

In fact, if we regard $V$ as a subvariety of itself then we find that the vector space $\mathcal{X}(V / V)$ is of dimension zero (for $Q(V / V)$ is the entire field $\mathcal{f}(V)$ and $\boldsymbol{m}(V / V)$ is the zero ideal). Hence by Definition 1 (where we have now: $W=V, \rho=r=\operatorname{dim} V), V$ itself is simple for $V$.

Thus Theorem 3 implies that the singular manifold of an irreducible $V$ is a proper subset of $V$. In the course of the proof of Theorem 3 we shall actually establish the following stronger result: if $W$ is any proper algebraic subvariety of $V$, then $V-W$ carries at least one simple point of $V$ (see Lemma 4 below). That is as far as we were able to go by local arguments. For the proof that the singular manifold of $V$ is algebraic we have to fall back on the global algebraic criterion for simple loci ( $\$ 9.6)$.

We proceed to prove Theorem 3 under the assumption that $V$ is irreducible. The generalization to arbitrary varieties $V$ will then be straightforward. 
4.3. Proof of Theorem 3. We first show that the condition stated in Theorem 3 is necessary. We assume therefore that $W$ contains a simple point of $V$, and we must prove that then $W$ itself is simple for $V$. Let $P$ be a point of $W$ which is simple for $V$ and let $\rho$ be the dimension of $W$. Suppose that our assertion that $W$ is simple for $V$ has already been proved in the case when $W$ is a curve $(\rho=1)$. We then show that the assertion follows by induction with respect to $\rho$. We fix on $W$ an irreducible subvariety $W_{1}$ passing through $P$ and of dimension $\rho-1$. By our induction we have then that $W_{1}$ is a simple subvariety of $V$. By a reduction to dimension zero we replace $W_{1}$ by a point $W_{1}^{*}$ over a suitable extension $\kappa^{*}$ of the ground field $\kappa$. By this reduction the varieties $W$ and $V$ are replaced respectively by a curve $W^{*}$ and by a variety $V^{*}$ of dimension $r-\rho+1$ over $\kappa^{*}$. We have $W_{1}^{*} \subset W^{*} \subseteq V^{*}$ and $Q\left(W_{1}^{*} / V^{*}\right)=Q\left(W_{1} / V\right)$. Hence, since $W_{1}$ is simple for $V$, the point $W_{1}^{*}$ is simple for $V^{*}$. Since the point $W_{1}^{*}$ belongs to the curve $W^{*}$ and since we have assumed that the case $\rho=1$ has already been settled, it follows that $W^{*}$ is a simple curve on $V^{*}$. But then also $W$ is simple for $V$, since $Q(W / V)=.Q\left(W^{*} / V^{*}\right)$.

Suppose now that $W$ is a curve. We shall first consider the case in which the point $P$ is simple not only for $V$ but also for $W$. We apply Lemma 2 of $\$ 2.3$ taking for $V^{\prime}$ and $W$ respectively the curve $W$ and the point $P$. In the present case we have $\operatorname{dim} \mathscr{H}(P / W)=1$ (since $P$ is simple for $W$ ) and $\operatorname{dim}$ $\mathcal{H}(P / V)=r=$ dimension of $V$. Hence the dimension of the space annihilated by the linear transformation $\phi$ of $\mathcal{H}(P / V)$ onto $\mathscr{H}(P / W)$ must be $r-1$. This means that the ideal $p(W / V)$ must contain $r-1$ elements $u_{i}$ such that the vectors $\tau u_{i}$ in $\mathscr{H}(P / V)$ are independent. By Lemma 3, the $r-1$ elements $u_{1}, u_{2}, \cdots, u_{r-1}$ generate a prime one-dimensional ideal in $Q(P / V)$, and the corresponding curve through $P$ must therefore coincide with $W$. Hence by the corollary to Lemma $3, W$ is simple for $V$.

There remains the case in which $P$ is a singular point of the curve $W$. In this case we apply to $V$ successive quadratic transformations the effect of which is to resolve the singularity $P$ of the curve $W$. Let $V^{\prime}$ and $W^{\prime}$ be respectively the transforms of $V$ and $W$ under the product of the successive quadratic transformations. Since the center of each quadratic transformations is a point, the quotient ring $Q(W / V)$ is not affected by these transformations, that is, we have $Q(W / V)=Q\left(W^{\prime} / V^{\prime}\right)$. On the other hand $W^{\prime}$ carries at least one point $P^{\prime}$ of $V^{\prime}$ which is simple for both $V^{\prime}$ and $W^{\prime}$, namely any of the points of $W^{\prime}$ into which the singular points $P$ of $W$ has been resolved. Consequently, by the preceding case, $W^{\prime}$ is simple for $V^{\prime}$. Since $Q(W / V)=Q\left(W^{\prime} / V^{\prime}\right)$, it follows that also $W$ is simple for $V$.

4.4. Continuation of the proof. The sufficiency of the condition stated in Theorem 3 is included in the following lemma:

LEMмA 4. If $W$ is an irreducible simple subvariety of $V$, of dimension $\rho$, $0 \leqq \rho \leqq r=\operatorname{dim} V$, and if $S$ is a proper algebraic subvariety of $W$, then $W-S$ contains at least one simple point of $V$. 
Proof of the lemma. We shall prove this lemma by induction with respect to $r$. If $r=1, V$ is a curve, and there exists then at most a finite number of points $P$ on $V$ such that $Q(P / V)$ is not integrally closed. For all other points $P$ of $V$ the ring $Q(P / V)$ is a discrete valuation ring, $m(P / V)$ is a principal ideal, and therefore $P$ is a simple point of $V$. What we have shown is that a curve $V$ has at most a finite number of singular points, and this establishes the lemma for $r=1$ (the permissible values of $\rho$ being 0 and 1 ).

We now assume that the lemma is true for varieties $V$ of dimension less than $r$ and we proceed to prove the lemma for a given variety $V$ of dimension $r$.

We first consider the case $\rho<r$. We select in $R=R[V]$ a set of $r-\rho$ uniformizing parameters $t_{1}, t_{2}, \cdots, t_{r-\rho}$ of $W$, and we consider the ideal $R \cdot\left(t_{1}, t_{2}, \cdots, t_{r-\rho}\right)$ and a normal decomposition of this ideal in primary components. By the definition of uniformizing parameters and by known relations between ideals in $R$ and in the quotient ring $Q(W / V)$, the prime ideal $\mathfrak{p}=\boldsymbol{p}(W / V)$ must be itself one of the primary (necessarily isolated) components of the ideal $R \cdot\left(t_{1}, t_{2}, \cdots, t_{r-\rho}\right)$. Let $\mathfrak{q}_{1}, \mathfrak{q}_{2}, \cdots, \mathfrak{q}_{h}$ be the other primary components, and let $\mathfrak{p}_{1}, \mathfrak{p}_{2}, \cdots, \mathfrak{p}_{h}$ be their associated prime ideals. Let $W_{i}$ be the irreducible subvariety of $V$ defined by $\mathfrak{p}_{i}$. Since $\mathfrak{p}_{i} \nsubseteq \mathfrak{p}$, we have $W_{i} \nsupseteq W$, and therefore if we denote by $S_{1}$ the intersection of $W$ with the variety $\bigcup_{i=1}^{h} W_{i}$, then $S_{1}$ is a proper algebraic subvariety of $W$. Therefore also $S \cup S_{1}$ is a proper algebraic subvariety of $W$. Since $\rho<r$, it follows by our induction that there exists at least one point on $W$ which is simple for $W$ and does not belong to $S \cup S_{1}$. We shall now prove that any simple point of $W$ which does not belong to $S_{1}$ is necessarily a simple point of $V$. This will establish the lemma for varieties $V$ of dimension $r$, provided $\rho<r$.

Let then $P$ be a point of $W$ such that $P \notin S_{1}$. Since $P \notin W_{i}, i=1,2, \cdots, h$, we can find an element $\omega_{i}$ in $q_{i}$ such that $\omega_{i} \notin m(P / V)$. If $\omega=\prod_{i=1}^{h} \omega_{i}$ then $\omega$ is a unit in $Q(P / V)$ and we have moreover $\omega \cdot \mathfrak{p} \subseteq R \cdot\left(t_{1}, t_{2}, \cdots, t_{r-\rho}\right)$. Since $t_{i} \in \mathfrak{p}$ this shows that $\left(t_{1}, t_{2}, \cdots, t_{r-\rho}\right)$ is a local $P$-basis of $\mathfrak{p}$. We now use Lemma 2 (where $W, V^{\prime}$, and $V$ are now respectively $P, W$, and $V$ ). The subspace of $\mathcal{H}(P / V)$ which is spanned by the vectors belonging to $\tau \mathfrak{p}$ is in this case spanned by the $r-\rho$ vectors $\tau t_{i}$. Hence $\operatorname{dim} \mathcal{H}(P / V) \leqq r-\rho$ $+\operatorname{dim} \mathcal{H}(P / W)$. Since $P$ is simple for $W$, we have $\operatorname{dim} \mathcal{H}(P / W)=\rho$, and hence $\operatorname{dim} \mathcal{X}(P / V) \leqq r$. Hence necessarily $\operatorname{dim} \mathcal{X}(P / V)=r$, and $P$ is a simple point of $V$, as was asserted.

There remains to be considered the case $\rho=r$, that is, $W=V$. The consideration of the conductor of the coordinate ring $R=R[V]$ with respect to the integral closure of $R$ in its quotient field $\mathcal{F}(V)$ shows that there exists at most a finite number of $(r-1)$-dimensional irreducible subvarieties $H$ of $V$ such that the quotient ring $Q(H / V)$ is not integrally closed. There exist therefore irreducible subvarieties $H$ of $V$, of dimension $r-1$, such that $Q(H / V)$ is integrally closed and $H \nsupseteq S$. Let $H_{0}$ be such a subvariety of $V$. Since $Q\left(H_{0} / V\right)$ 
is integrally closed, this quotient ring is a discrete valuation ring and therefore $H_{0}$ is simple for $V$. Since $H_{0} \nsupseteq S, H_{0} \cap S$ is a proper algebraic subvariety of $H_{0}$. By the preceding case $\rho<r$ (namely $\rho=r-1$ ) we can find at least one point $P$ on $H_{0}$ which is simple for $V$ and does not belong to $H_{0} \cap S$. We have thus shown that there exist simple points of $V$ which are not in $S$. This completes the proof of Theorem 3 for an irreducible $V$.

4.5. The case of a reducible variety. The case of a reducible $V$ can now be readily taken care of. Let $V_{1}, V_{2}, \cdots, V_{o}$ be the irreducible components of $V$. We assume first that $W$ contains a simple point $P$ of $V$. Then $P$ belongs to only one of the irreducible components $V_{i}$, say to $V_{1}$, and $P$ is necessarily a simple point of $V_{1}$ (Definition 2, §3.4). From $P \notin V_{i}, i=2,3, \cdots, g$, follows $W \Phi V_{i}$ whence $W \subseteq V_{1}$. Since $W$ carries the simple point $P$ of $V_{1}$, it follows, by the irreducible case of Theorem 3, that $W$ is simple for $V_{1}$. Thus $W$ belongs to only one irreducible component of $V$ and is simple for that component. Hence $W$ is simple for $V$.

Assume now that $W$ is simple for $V$. For a suitable labeling of the irreducible components $V_{i}$ of $V$ we may assume that $W \subseteq V_{1}, W \Phi V_{i}, i=2,3, \cdots, g$, and that $W$ is simple for $V_{1}$. Let $S$ be the intersection of $W$ with $\bigcup_{i=2}^{0} V_{i}$. Then $S$ is a proper algebraic subvariety of $W$, and therefore by Lemma 4 there exists a point $P$ on $W-S$ which is simple for $V_{1}$. This point $P$ does not belong to any other component $V_{i}, i \neq 1$. Hence $P$ is simple for $V$. This completes the proof of Theorem 3 .

\section{Simple loci and regular rings.}

5.1. The identity of the two concepts. A special class of local rings, called regular, has been first introduced and studied by Krull [7], and the theory of these rings has been further developed by Chevalley [2] and I. S. Cohen $[3]\left({ }^{4}\right)$. Let $\mathfrak{o}$ be a local ring, $\mathfrak{m}$ the maximal ideal in $\mathfrak{D}$, and let $\left(t_{1}, t_{2}, \cdots, t_{8}\right)$ be a minimal basis of $\mathfrak{m}$. The local ring $\mathfrak{o}$ is called regular if the following condition is satisfied: in any homogeneous relation $f\left(t_{1}, t_{2}, \cdots, t_{s}\right)=0$ between $t_{1}, t_{2}, \cdots, t_{s}$, with coefficients in $\mathfrak{0}$, the coefficients necessarily all belong to $\mathrm{m}$. An equivalent condition is the following: if $\phi\left(t_{1}, t_{2}, \cdots, t_{8}\right) \in \mathfrak{m}^{\nu+1}, \phi=a$ form of degree $\nu$ with coefficients in $\mathfrak{D}$, then all the coefficients of $\phi$ must belong to $\mathrm{m}$. That the second of these two conditions implies the first is trivial. That the first condition implies the second follows immediately from the fact that every element of $\mathrm{m}^{\nu+1}$ can be expressed as a form of degree $\nu+1$ in $t_{1}, t_{2}, \cdots, t_{s}$ with coefficients in $\mathfrak{D}$, and hence also as a form of degree $\nu$ in $t_{1}, t_{2}, \cdots, t_{s}$, with coefficients in $\mathrm{m}$.

If $\mathfrak{D}$ is an arbitrary local ring, then the ring $\mathfrak{m} / \mathfrak{m}^{2}$, as an additive group, can be regarded as a vector space over the residue field $\Delta=\mathfrak{o} / \mathrm{m}$ as field of scalars. We denote this vector space by $\mathscr{X}_{1}$. The dimension of $\mathscr{X}_{1}$ is equal to the number $s$ of elements $u_{1}, u_{2}, \cdots, u_{s}$ in any minimal basis of $m$. More

(4) The term "regular" is due to Chevalley. The original term used by Krull was " $p$-Reihenringen." 
generally, $\mathfrak{m}^{\nu} / \mathfrak{m}^{\nu+1}$ can be regarded as a vector space over $\Delta$. We shall denote this space by $\mathcal{X}_{\nu}$. The $C_{s+\nu-1, \nu}$ power products $u_{1}^{i_{1}} u_{2}^{i_{2}} \cdots u_{s}^{i_{s}}$ of degree $\nu$, reduced modulo $\mathrm{m}^{\nu+1}$, form a basis of $\mathcal{X}_{\nu}$, but not necessarily an independent one. The ring $\mathrm{o}$ is regular if and only if the above basis is independent, that is, if and only if $\operatorname{dim} \mathscr{X}_{\nu}=C_{s+\nu-1, \nu}$ for all positive integers $\nu$.

THEOREM 4. Given an irreducible algebraic variety $V$, an irreducible algebraic subvariety $W$ of $V$ is simple for $V$ if and only if the quotient ring $Q(W / V)$ is regular.

Proof. By the reduction to dimension zero (\$2.2) it is sufficient to prove the theorem for points of $V$. We assume then that $W=P=$ a point of $V$. Let $\mathrm{D}=Q(P / V), \mathfrak{m}=\boldsymbol{m}(P / V), s=\operatorname{dim} \mathcal{H}(P / V)$, and $r=\operatorname{dim} V$. We know that $s \geqq r$. We have to show that $s=r$ if and only if $\mathrm{D}$ is a regular ring.

(1) Assume $s>r$. Let $\left(t_{1}, t_{2}, \cdots, t_{s}\right)$ be a minimal basis of $\mathrm{m}$. It is always possible to choose $r$ elements in that basis in such a fashion that the o-ideal generated by these elements is of dimension 0 . We may assume that $t_{1}, t_{2}, \cdots, t_{r}$ are such elements. Then the $\mathrm{o}$-ideal $\mathrm{q}=0 \cdot\left(t_{1}, t_{2}, \cdots, t_{r}\right)$ is primary, and its associated prime ideal is $\mathrm{m}$. Since $t_{s} \in \mathfrak{m}$, some power of $t_{s}$, say $t_{s}^{h}$, belongs to $q$. There exists an integer $\nu \geqq 0$ such that $t_{s}^{h} \in q m^{\nu}, t_{s}^{h} \in q m^{\nu+1}$ (such an integer exists since $\bigcap_{i=1}^{\infty} \mathfrak{m}^{i}=(0)$ and $t_{s} \neq 0$; by $\mathfrak{q} \cdot \mathfrak{m}^{0}$ we mean $\mathfrak{q}$ ). Every element of $\mathfrak{m}^{\nu}$ is expressible as a form of degree $\nu$ in $t_{1}, t_{2}, \cdots, t_{s}$ with coefficients in $\mathfrak{D}$, while every element in $\mathfrak{q}$ is expressible as a linear form in $t_{1}, t_{2}, \cdots, t_{r}$ with coefficients in $\mathfrak{D}$. Since $t_{s} \in \mathfrak{q m}^{\nu}$, we therefore have a relation of the form:

$$
F(t)=t_{s}{ }^{h}-\phi_{1}\left(t_{1}, t_{2}, \cdots, t_{r}\right) \psi_{\nu}\left(t_{1}, t_{2}, \cdots, t_{s}\right)=0,
$$

where $\phi_{1}$ and $\psi_{\nu}$ are forms with coefficients in $\mathfrak{0}$, of degrees 1 and $\nu$ respectively. If $h<\nu+1$ then the relation $t_{s}^{h} \in \mathfrak{q m}^{\nu}$ implies $t_{s}^{h} \in \mathfrak{m}^{\nu+1} \subseteq \mathfrak{m}^{h+1}$, and therefore $\mathrm{D}$ is not regular. Assume now that $h \geqq \nu+1$. Since $t_{s}^{h} \notin q \mathfrak{m}^{\nu+1}$ the coefficients of $\phi_{1}$ as well as the coefficients of $\psi_{\nu}$ are not all in $\mathrm{m}$. If $h>\nu+1$ then we have $\phi_{1}(t) \psi_{\nu}(t)=t_{s}^{h} \in \mathfrak{m}^{h} \subseteq \mathfrak{m}^{\nu+2}$, where, as we have just seen, $\phi_{1} \psi_{\nu}$ is a form of degree $\nu+1$ in $t_{1}, t_{2}, \cdots, t_{s}$ whose coefficients are not all in $m$. Hence again $\mathrm{D}$ is not regular. If finally $h=\nu+1$ then $F(t)$ is a form of degree $h$ in $t_{1}, t_{2}, \cdots, t_{s}$, and since the product $\phi_{1} \psi_{\nu}$ does not contain a term depending on $t_{s}$ only $\left(\phi_{1}\right.$ is linear homogeneous in $\left.t_{1}, t_{2}, \cdots, t_{r}\right)$, it follows that the coefficient of $t_{s}^{h}$ in $F(t)$ is 1 . The relation $F(t)=0$ shows then that $\mathrm{D}$ is not regular. We have therefore shown that if $s>r$ then the ring $\mathrm{D}$ is not regular.

(2) Assume $s=r$. To prove that in this case $\mathfrak{D}$ is a regular ring we first consider the case in which the residue field $\Delta(=\mathfrak{o} / \mathfrak{m})$ is infinite.

If $f\left(z_{1}, z_{2}, \cdots, z_{r}\right)$ is a form in indeterminates $z_{i}$ with coefficients in $\mathfrak{D}$ we shall denote by $\bar{f}\left(z_{1}, z_{2}, \cdots, z_{r}\right)$ the form with coefficients in $\Delta$ which is obtained when the coefficients of $f$ are replaced by their $\mathfrak{m}$-residues in $\Delta$. To show that $\mathrm{D}$ is regular we have to show the following: if $f\left(t_{1}, t_{2}, \cdots, t_{r}\right)=0$ then $\bar{f}\left(z_{1}, z_{2}, \cdots, z_{r}\right)=0$. We consider an arbitrary linear nonsingular homo- 
geneous transformation

$$
z_{i}^{\prime}=\sum_{j=1}^{r} \bar{a}_{i j} z_{j}, \quad i=1,2, \cdots, r ; \bar{a}_{i j} \in \Delta .
$$

With any such transformation we can associate (in more than one way) a transformation

$$
t_{i}^{\prime}=\sum_{j=1}^{r} a_{i j} t_{j}, \quad i=1,2, \cdots, r ; a_{i j} \in \mathrm{o},
$$

of the given minimal basis $\left(t_{1}, t_{2}, \cdots, t_{r}\right)$ of $\mathfrak{m}$ into another minimal basis $\left(t_{1}^{\prime}, t_{2}^{\prime}, \cdots, t_{r}^{\prime}\right)$ of $\mathrm{m}$. We have only to take for the $a_{i j}$ elements of $\mathfrak{o}$ whose m-residues are the $\bar{a}_{i j}$ (that the $t^{\prime \prime}$ s also form a basis of $\mathfrak{m}$ follows from the assumption $\left|\bar{a}_{i j}\right| \neq 0$, which implies $\left|a_{i j}\right| \notin \mathfrak{m}$, that is, $\left|a_{i j}\right|$ is a unit in $\mathfrak{D}$ ). It is clear that if $f\left(t_{1}, t_{2}, \cdots, t_{r}\right)$ is a form in the $t^{\prime}$ s with coefficients in 0 , and if $\phi\left(t_{1}^{\prime}, t_{2}^{\prime}, \cdots, t_{r}^{\prime}\right)$ is the transform of $f(t)$ under the transformation (4), then $\bar{\phi}\left(z_{1}^{\prime}, z_{2}^{\prime}, \cdots, z_{r}^{\prime}\right)$ is the transform of $\bar{f}\left(z_{1}, z_{2}, \cdots, z_{r}\right)$ under the transformation (3). Given $f\left(t_{1}, t_{2}, \cdots, t_{r}\right)$, homogeneous of degree $\nu$, and assuming that $\bar{f}\left(z_{1}, z_{2}, \cdots, z_{r}\right) \neq 0$, we can find a linear transformation (3) such that in $\phi\left(z_{1}^{\prime}, z_{2}^{\prime}, \cdots, z_{r}^{\prime}\right)$ the coefficient of $z_{r}^{\prime v}$ is not equal to 0 (since $\Delta$ is an infinite field). We then can match this transformation by a corresponding transformation (4) in such a fashion that the coefficient of $t_{r}^{\prime}{ }^{\nu}$ in $\phi\left(t_{1}^{\prime}, t_{2}^{\prime}, \cdots, t_{r}^{\prime}\right)$ is not in $\mathfrak{m}$, hence is a unit in $\mathbf{D}$. The upshot of the preceding argument is then this: if we have a homogeneous relation $f\left(t_{1}, t_{2}, \cdots, t_{r}\right)=0$ of degree $\nu$ between the $t$ 's, with coefficients in $\mathrm{D}$, and if we assume that $\bar{f}\left(z_{1}, z_{2}, \cdots, z_{r}\right) \neq 0$ we can assume (by first changing, if necessary, the minimal basis of $m$ ) that the coefficient of $t_{r}^{\nu}$ in $f$ is a unit $e$ in $\mathrm{o}$. But then the relation $f(t)=0$ implies that $t_{r}^{\nu} \in \mathcal{D} \cdot\left(t_{1}, t_{2}, \cdots, t_{r-1}\right), \mathfrak{m}^{\nu} \subseteq \mathcal{D} \cdot\left(t_{1}, t_{2}, \cdots, t_{r-1}\right)$, whence the ideal $\mathfrak{D} \cdot\left(t_{1}, t_{2}, \cdots, t_{r-1}\right)$ is zero-dimensional (having $\mathfrak{m}$ as associated prime ideal). This is impossible, since all minimal primes of the ideal $\mathrm{D} \cdot\left(t_{1}, t_{2}, \cdots, t_{r-1}\right)$ are of dimension not less than $r-(r-1)=1$. This contradiction has been obtained because we have assumed that $\bar{f}\left(z_{1}, z_{2}, \cdots, z_{r}\right) \neq 0$. Hence we must have $\bar{f}\left(z_{1}, z_{2}, \cdots, z_{r}\right)=0$ whenever $f\left(t_{1}, t_{2}, \cdots, t_{r}\right)=0$, and this shows that $D$ is a regular ring.

If $\Delta$ is a finite field, we adjoin to the field $\mathcal{F}(V)$ an indeterminate $u$ and we take as new ground field the field $\kappa^{*}=\kappa(u)$. We denote by $V^{*}$ and $P^{*}$ respectively the variety over $\kappa^{*}$ having the same general point as $V$ and the point over $\kappa^{*}$ having the same coördinates as $P$. We have $\operatorname{dim} V^{*} / \kappa^{*}=\operatorname{dim} V / \kappa=r$, $R^{*}=R\left[V^{*}\right]=\kappa^{*} R$ (where $R=R[V]$ ) and $\boldsymbol{p}\left(P^{*} / V^{*}\right)=R^{*} \cdot \boldsymbol{p}(P / V)$. From these facts it follows immediately that if $0^{*}=Q\left(P^{*} / V^{*}\right)$ and $\mathfrak{m}^{*}=m\left(P^{*} / V^{*}\right)$, then

$$
\begin{aligned}
\mathfrak{m}^{*} & =\mathfrak{o}^{*} \mathfrak{m}, \\
\mathfrak{m} & =\mathfrak{m}^{*} \cap \mathrm{o} .
\end{aligned}
$$


From (5) it follows that our minimal basis $\left(t_{1}, t_{2}, \cdots, t_{r}\right)$ of $\mathfrak{m}$ is also a basis of $\mathfrak{m}^{*}$-necessarily minimal, since $\operatorname{dim} V^{*}=r$. The residue field $\Delta^{*}=\mathfrak{o}^{*} / \mathfrak{m}^{*}$ contains the infinite new ground field $\kappa^{*}$. Hence, by the preceding proof, $0^{*}$ is a regular ring. Therefore if $f\left(t_{1}, t_{2}, \cdots, t_{r}\right)=0$ is a homogeneous relation with coefficients in $\mathfrak{D}$, these coefficients are all in $\mathrm{m}^{*}$. But then, in view of $\left(5^{\prime}\right)$, these coefficients must all belong to $\mathrm{m}$. This completes the proof of the theorem.

5.2. Unique local factorization at simple loci. The regular rings which occur in algebraic geometry as quotient rings of simple subvarieties are "unramified" in the sense of I. S. Cohen [3, p. 88], for they contain a field (namely the ground field $\kappa$ ). The theorems proved by Cohen for unramified regular rings are therefore valid for quotient rings of simple subvarieties. Thus if $W$ is an irreducible $\rho$-dimensional simple subvariety of an irreducible $r$-dimensional variety $V$ and if $\mathfrak{D}=Q(W / V)$, then the completion $\mathrm{o}^{*}$ of $\mathrm{o}$ (with respect to the powers of the maximal ideal $m$ in $\mathfrak{b}$ ) is isomorphic to the ring of formal power series in $r-\rho$ indeterminates over the residue field $\Delta=0 / \mathfrak{m}[3$, Theorem 15 , p. 88], and therefore [3, Theorem 18, p. 94], $\mathrm{o}^{*}$ is a unique factorization domain. The following result is a consequence of a general theorem on local rings due to Chevalley: if $W$ is an arbitrary (not necessarily simple) irreducible subvariety of $V$, then any prime ideal $p$ in the quotient ring $\mathrm{o}=Q(W / V)$ is unramified in the completion $\mathrm{o}^{*}$ of $\mathfrak{0}$, that is, $\mathrm{D}^{*} \cdot \mathfrak{p}$ is a finite intersection of prime ideals in $\mathrm{o}^{*}$ (see $\mathrm{C}$. Chevalley, Intersections of algebraic and algebroid varieties, Trans. Amer. Math. Soc. vol. 57 (1945) p. 9, last sentence of Lemma 9, and p. 11, Theorem 1). Using this result and the fact that $0^{*}$ is a unique factorization domain, we can now prove the following theorem:

THEOREM 5. The quotient ring of a simple subvariety is a unique factorization domain.

Proof. Let $\mathfrak{p}$ be a minimal prime ideal in $\mathfrak{o}$. To prove the theorem we have only to show that $\mathfrak{p}$ is a principal ideal. Let

$$
\mathfrak{p}^{*} \cdot \mathfrak{p}=\bigcap_{i=1}^{h} \mathfrak{p}_{i}^{*},
$$

where we may assume of course that no $\mathfrak{p}_{i}{ }^{*}$ is superfluous. It has been shown by Chevalley [2, Propositions 5 and 6, p. 699] for the completion $0^{*}$ of an arbitrary local ring $\mathfrak{D}$ that if $\mathfrak{p}$ is a prime ideal in $\mathfrak{D}$ then every prime ideal of $\mathfrak{o}^{*} \cdot \mathfrak{p}$ contracts to $\mathfrak{p}$. Using this result, we have therefore: $\mathfrak{p}_{i}^{*} \cap_{\mathfrak{o}}=\mathfrak{p}$, $i=1,2, \cdots, h$.

We now show that each prime ideal $\mathfrak{p}_{i}{ }^{*}$ is minimal in $\mathbf{0}$. Consider for instance the ideal $p_{1}^{*}$. Let $\omega$ be a fixed element of $p, \omega \neq 0$. Since $\omega \in p_{1}^{*}$, some isolated prime ideal of $\mathfrak{o}^{*} \cdot \omega$ must be contained in $p_{1}^{*}$. Let $p^{*}$ be such an isolated prime ideal of $\mathfrak{p}^{*} \cdot \omega, \mathfrak{p}^{*} \subseteq \mathfrak{p}_{1}^{*}$. We have $\mathfrak{p}^{*} \cap \mathcal{D} \subseteq \mathfrak{p}_{1}^{*} \cap \mathcal{D}=\mathfrak{p}$ and $\mathfrak{p}^{*} \cap \mathfrak{D} \neq(0)$ since $\omega \in p^{*}$. Hence $p^{*} \cap \mathfrak{D}=\mathfrak{p}$ since $\mathfrak{p}$ is minimal in $\mathfrak{o}$. Therefore $\mathfrak{o}^{*} \mathfrak{p} \subseteq \mathfrak{p}^{*}$ and conse- 
quently $\mathfrak{p}^{*}$ must contain one of the prime ideals $\mathfrak{p}_{i}^{*}, i=1,2, \cdots, h$. However, since we know already that $p^{*}$ in its turn is contained in $p_{1}^{*}$ and since $p_{1}^{*}$ is not superfluous in (6), we conclude that $p^{*}=p_{1}^{*}$. Hence $p_{1}^{*}$ is an isolated prime ideal of the principal ideal $0^{*} \cdot \omega$, and therefore it is necessarily minimal in $D^{*}$, as was asserted.

So far we have made no use of the assumption that $\mathrm{o}$ is the quotient ring of a simple subvariety. Using this assumption we know then that $\mathfrak{D}^{*}$ is a unique factorization domain. Therefore the ideal $0^{*} \cdot p$ must be a principal ideal, since we have just proved that $\boldsymbol{D}^{*} \cdot p$ is the intersection of minimal prime ideals. Hence $p$ must contain an element $u$ such that $p^{*} \cdot p=0^{*} \cdot u$. It is known that every ideal $\mathfrak{A}$ in $\mathfrak{D}$ satisfies the relation $\mathfrak{D}^{*} \cdot \mathfrak{A} \cap \mathfrak{D}=\mathfrak{A}$ (Krull $[7$, Theorem 15]). Hence $\mathfrak{p}=\mathfrak{o}^{*} \cdot \mathfrak{p} \cap \mathfrak{D}=\mathfrak{o}^{*} \cdot u \cap \mathfrak{D}=\mathfrak{D} \cdot u$. This completes the proof.

5.3. The abstract analogue of Theorem 3 and an example of F. K. Schmidt. We shall conclude this section with a discussion of the first part of the proof of Theorem $3(\$ 4.3)$ from the standpoint of the general theory of regular local rings. The necessity of the condition stated in Theorem 3 is a strictly local fact. For let $P$ be a point of $W$, let $\mathrm{D}=Q(P / V)$ and let $\mathfrak{p}$ be the prime ideal in o defined by $W(\mathfrak{p}=\mathfrak{p} \cdot \boldsymbol{p}(W / V))$. Then in view of Theorem 4 of $\S 5.1$, the "only if" part of Theorem 3 can be stated as follows: "if $\mathfrak{D}$ is a regular ring, then also the ring $\mathrm{o}_{\mathfrak{p}}$ (that is, $Q(W / V)$ ) is regular." The question arises whether this statement is true for an arbitrary regular local ring $D$ and for any prime ideal $\mathfrak{p}$ in $\mathfrak{D}$ (the statement is trivial for minimal prime ideals $\mathfrak{p}$ in view of the fact that any regular local ring is an integrally closed domain; see [7, Theorem 6]). At present it is only known that the statement is true if the regular ring $D$ is complete (Cohen [3, Theorem 20, p. 97]). In addition to calling attention to this unsolved question, we wish to analyze the proof of the "only if" part of Theorem 3 in order to point out why that proof fails in the general case where we have an arbitrary regular local ring $\mathfrak{D}$. The first part of that proof consists in a reduction to the case $\operatorname{dim} W=\rho=1$, and that reduction can be carried out just as it stands also in the general case. In the second part of the proof we have first considered the case in which $P$ is a simple point of $W$. The corresponding assumption in the general case can be expressed by saying that the residue class ring $\mathfrak{D} / \mathfrak{p}$ is a regular ring (hence a discrete valuation ring, since $\mathfrak{p}$ is of dimension 1 ). Also in this case our proof of the regularity of $o_{p}$ remains valid in the general case.

The critical phase in our proof is that in which the local ring $\mathfrak{o} / \mathfrak{p}$ (of dimension 1) is not regular; in other words: $\mathfrak{o} / \mathfrak{p}$ is a primary integral domain which is not integrally closed. In this case we have applied to the neighborhood of the point $P$ on $V$, that is, to the local ring $\mathfrak{D}$, successive quadratic transformations, the effect of which was to resolve the singularity of the curve $W$ at $P$, that is, to transform $\mathfrak{D} / \mathrm{p}$ into a regular ring. It is this step that may fail to work in the general case. The following free presentation of an example by F. K. Schmidt [9] will illustrate this possibility. 
Let $\kappa$ be a field of characteristic $p \neq 0$ and let $\Sigma^{\prime}$ be the field $\kappa(x, t)$ in two independent variables $x$ and $t$ over $\kappa$. Let $\xi_{1}, \xi_{2}, \ldots, \xi_{n}, \ldots$ be an infinite sequence of elements of $\kappa$ such that the formal power series

$$
\xi_{1}+\xi_{2} x+\xi_{3} x^{2}+\cdots
$$

is transcendental over $\kappa(x)(\mathfrak{b})$. Then the analytical branch in the $(x, t)$-plane defined by

$$
t=\left(\xi_{1}+\xi_{2} x+\cdots\right)^{p}
$$

is not algebraic, and therefore it defines a zero-dimensional, discrete, rank 1 valuation $v^{\prime}$ of $\Sigma^{\prime}$. Let $\boldsymbol{D}^{\prime}$ be the valuation ring of $v^{\prime}$. We now consider the field $\Sigma=\Sigma^{\prime}(\tau)=\kappa(x, \tau)$, where $\tau=t^{1 / p}$, and the ring

$$
\mathfrak{D}=\mathfrak{o}^{\prime}[\tau]=\mathfrak{D}^{\prime} \cdot 1+\mathfrak{D}^{\prime} \cdot \tau+\cdots+\mathfrak{o}^{\prime} \cdot \tau^{p-1} .
$$

Let $v$ be the extension of $v^{\prime}$ to $\Sigma$ (the extension is unique, for $\Sigma$ is purely inseparable over $\left.\Sigma^{\prime}\right)$. It is clear by (7) that $v$ is defined by the following analytical branch in the $(x, \tau)$-plane:

$$
\tau=\xi_{1}+\xi_{2} x+\cdots
$$

Since $\boldsymbol{D}^{\prime}$ is a discrete, rank 1, valuation ring, it is a local (regular) ring of dimension 1. Since $\mathfrak{D}$ is a finite $\mathfrak{D}^{\prime}$-module, it follows that also $\mathfrak{D}$ is a local ring of dimension 1 (Chevalley [2, Proposition 3, p. 694]). We shall now show that $\mathrm{D}$ is not regular and that it cannot be transformed into a regular ring by successive quadratic transformations.

Let $\tau_{1}=\left(\tau-\xi_{1}\right) / x$. We have, by $(9), v\left(\tau_{1}\right) \geqq 0$, whence $v^{\prime}\left(\tau_{1}^{p}\right) \geqq 0$. Consequently $\tau_{1}^{p} \in \mathfrak{o}^{\prime}$ and therefore $\tau_{1}$ is integrally dependent on $\mathfrak{D}$. However $\tau_{1}=-\xi_{1} / x \cdot 1+1 / x \cdot \tau$, whence, by $(8), \tau_{1} \notin 0$, since $1, \tau, \cdots, \tau^{p-1}$ form an independent basis of $\Sigma$ over $\Sigma^{\prime}$ and since $1 / x \in 0^{\prime}$. Consequently 0 is not integrally closed and therefore it cannot be a regular ring.

The maximal ideal $\mathrm{m}^{\prime}$ in $\boldsymbol{D}^{\prime}$ is the principal ideal $\mathfrak{o}^{\prime} \cdot x$, since $v^{\prime}(x)=+1$. If $\mathfrak{m}$ denotes the maximal ideal in $\mathfrak{o}$, then we have $x \in \mathfrak{m}$, and also $\tau-\xi_{1} \in \mathfrak{m}$ (for $\left.\left(\tau-\xi_{1}\right)^{p}=t-\xi_{1}^{p} \in \mathfrak{m}^{\prime} \subseteq \mathfrak{m}\right)$. From (8) one sees then immediately that $x$ and $\tau-\xi_{1}$ form a basis for $\mathfrak{m}$.

To apply a "quadratic transformation" to the ring $\mathfrak{D}$ means to pass from $D$ to the following ring $\mathfrak{D}_{1}$ :

$$
\mathrm{D}_{1}=\mathrm{O}\left[\tau_{1}\right], \quad \tau_{1}=\left(\tau-\xi_{1}\right) / x .
$$

Let $t_{1}=\left(t-\xi_{1}^{p}\right) / x^{p}$. Then $\Sigma^{\prime}=\kappa(x, t)=\kappa\left(x, t_{1}\right)$ and, by $(7)$, the valuation $v^{\prime}$ can be equally well defined by the following analytical branch in the $\left(x, t_{1}\right)$ pláne:

(5) To construct such a power series one may proceed as Schmidt does, that is, take for $\kappa$ a field of infinite degree of transcendency over the prime field $\Gamma$ of characteristic $p$ and take for $\left(\xi_{1}, \xi_{2}, \cdots, \xi_{n}, \cdots\right)$ a transcendence set in $\kappa / \Gamma$. 


$$
t_{1}=\left(\xi_{2}+\xi_{3} x^{2}+\cdots\right)^{p} .
$$

We have $\tau_{1}=t_{1}^{1 / p}$ and $\mathfrak{o}_{1}=\mathfrak{o}^{\prime}\left[\tau_{1}\right]$. It follows that the ring $\mathfrak{D}_{1}$ is in similar relation to $\mathfrak{D}^{\prime}$ as $\mathfrak{D}$ was. This shows that $\mathfrak{D}_{1}$ is not a regular ring, and that the successive quadratic transformations will never lead to a regular ring (the next ring $\mathrm{D}_{2}$ will be defined as follows: $\mathrm{D}_{2}=\mathrm{D}_{1}\left[\tau_{2}\right]$, where $\tau_{2}=\left(\tau_{1}-\xi_{2}\right) / x$, and so on).

It is easy to verify that the integral closure of 0 in $\Sigma$ (that is, the valuation ring of $v$ ) is not a finite $\mathrm{p}$-module (Schmidt $[9, \mathrm{p} .447]$ ). This fact is the real reason why it is not possible to transform $D$ into a regular ring by successive "quadratic transformations." For it can be easily proved that "any primary integral domain $\boldsymbol{D}$ can be transformed into a regular ring by successive quadratic transformations, provided the integral closure of $\boldsymbol{D}$ is a finite $\boldsymbol{D}$-module."

\section{PART II. JACOBIAN CRITERIA FOR SIMPLE LOCI}

\section{The vector space $\mathcal{D}(W)$ of local differentials.}

6.1. The local $W$-differentials in $S_{n}$. Let $x_{1}, x_{2}, \cdots, x_{n}$ be coördinates in a linear $S_{n}$ and let $\left(\eta_{1}, \eta_{2}, \cdots, \eta_{n}\right)$ be the general point of an irreducible $\rho$-dimensional variety $W$ in $S_{n}$. If $u$ is any element of the maximal ideal $m$ of the quotient ring $\mathrm{D}$ of $W\left(\mathrm{D}=Q\left(W / S_{n}\right)\right)$, then $u=f(x) / g(x)$, where $f(x)$ and $g(x)$ are in $\kappa\left[x_{1}, x_{2}, \cdots, x_{n}\right], f(\eta)=0$ and $g(\eta) \neq 0$. From $g(\eta) \neq 0$ follows that the partial derivatives $\partial u / \partial x_{i}$ are elements of $\boldsymbol{0}$. The $\mathfrak{m}$-residues $\left(\partial u / \partial x_{i}\right)_{x=\eta}$ of these partial derivatives are therefore elements of the field $\Delta=\mathcal{F}(W)$ $=\kappa\left(\eta_{1}, \eta_{2}, \cdots, \eta_{n}\right)$. We call the ordered $n$-tuple

$$
\left(\partial u / \partial x_{1}, \partial u / \partial x_{2}, \cdots, \partial u / \partial x_{n}\right)_{x=\eta}
$$

of these residues the local differential of $u$ at $\dot{W}$, or the local $\dot{W}$-differential of $u$, and we denote it by $d_{W} u$. We emphasize our assumption that $u$ is an element of $\mathfrak{m}$, that is, $u=0$ on $W$.

We regard $d_{W} u$ as a vector in the $n$-dimensional vector space, over $\Delta$ as field of scalars, formed by all the ordered $n$-tuples of elements of $\Delta$. The set of all local differentials $d_{W} u$ ( $W$ is fixed, $u$ varies in $\mathfrak{m}$ ) is a linear subspace of that $n$-dimensional vector space. For in the first place, the relation $d_{W} u \pm d_{W} v=d_{W}(u \pm v)$ is obvious $(u, v \in \mathfrak{m}$ and consequently also $u \pm v \in \mathfrak{m})$. In the second place, if $\bar{\delta} \in \Delta$, we have $\bar{\delta}=\phi(\eta) / \psi(\eta)$, where $\phi(x), \psi(x)$ $\in \kappa\left[x_{1}, x_{2}, \cdots, x_{n}\right]$ and $\psi(\eta) \neq 0$. If $u \in \mathfrak{m}$ and if we set $\delta=\phi(x) / \psi(x)$, then $\delta u \in \mathfrak{m}$ and we have $d_{W}(\delta u)=\bar{\delta} \cdot d_{W} u$, for $(u)_{x=\eta}=0$.

We shall denote the vector space, over $\Delta$, formed by the local $W$-differentials $d_{W} u, u \in \mathfrak{m}$, by $\mathcal{D}(W)$.

6.2. The linear transformation $\mathcal{H}\left(W / S_{n}\right) \rightarrow \mathcal{D}(W)$. The given variety $W$ in $S_{n}$ defines also its local vector space $\mathcal{H}\left(W / S_{n}\right)$ over $\Delta(\$ 2.1)$, and we have the mapping $\tau$ of $\mathfrak{m}$ onto $\mathcal{H}\left(W / S_{n}\right)$ defined by (1). If for any $u$ in $\mathfrak{m}$ we let correspond to the vector $\tau u$ the local $W$-differential of $u$, we obtain a transformation 


$$
\tau u \rightarrow d_{W} u,
$$

$u \in \mathfrak{m}$,

of $\mathcal{X}\left(W / S_{n}\right)$ onto $\mathcal{D}(W)$. The linearity of this transformation is obvious. Therefore to prove that (10) is single-valued it is only necessary to show that if $\tau u=0$ then also $d_{W} u=0$. Now if $\tau u=0$, then $u \in \mathfrak{m}^{2}, u=\sum_{j=1}^{\nu} u_{j} v_{j}$, where $u_{j}, v_{j} \in \mathfrak{m}, j=1,2, \cdots, \nu$. Hence $\left(u_{j}\right)_{x=\eta}=\left(v_{j}\right)_{x=\eta}=0$, and therefore $\left(\partial u / \partial x_{i}\right)_{x=\eta}=0, i=1,2, \cdots, n$, that is, $d_{W} u=0$.

Hence (10) defines a linear transformation of $\mathcal{H}\left(W / S_{n}\right)$ onto $\mathcal{D}(W)$. We know that $W$ is simple for $S_{n}(\S 4)$, and that therefore $\mathcal{H}\left(W / S_{n}\right)$ is of dimension $n-\rho$. Consequently we can state the following result:

LEMMA 5. The dimension of the space $\mathcal{D}(W)$ of local $W$-differentials is at most equal to $n-\rho$, where $\rho$ is the dimension of $W$, and the dimension is exactly $n-\rho$ if and only if the linear transformation (10) is nonsingular.

\section{Jacobian criterion for simple points: the separable case.}

7.1. Criterion for uniformizing parameters. Let $P\left(\alpha_{1}, \alpha_{2}, \cdots, \alpha_{n}\right)$ be a point of $S_{n}$. We have constructed in $[13$, p. 541] a particular set of $n$ polynomials which form not only a minimal local basis (\$3.3) of the ideal $p(P)$ but even a basis of this ideal. It is a set of polynomials in $\kappa\left[x_{1}, x_{2}, \cdots, x_{n}\right]$,

$$
f_{1}\left(x_{1}\right), f_{2}\left(x_{1}, x_{2}\right), \cdots, f_{n}\left(x_{1}, x_{2}, \cdots, x_{n}\right),
$$

defined and uniquely determined by the following conditions:

a. $f_{i}$ depends only on $x_{1}, x_{2}, \cdots, x_{i}$ and is monic in $x_{i}$;

b. $f_{i}\left(\alpha_{1}, \alpha_{2}, \cdots, \alpha_{i}\right)=0$, that is, $f_{i}(x)=0$ at $P$;

c. $f_{i}\left(\alpha_{1}, \alpha_{2}, \cdots, \alpha_{i-1}, x_{i}\right)$ is irreducible over the field $\kappa\left(\alpha_{1}, \alpha_{2}, \cdots, \alpha_{i-1}\right)$;

d. If $f_{i}(x)$ is of degree $\nu_{i}$ in $x_{i}$ then for $j>i$ the degree of $f_{j}(x)$ in $x_{i}$ is less than $\nu_{i}$.

These $n$ polynomials $f_{i}(x)$ depend only on the order in which the indeterminates $x_{1}, x_{2}, \cdots, x_{n}$ are labeled. They shall be referred to as the canonical uniformizing parameters of $P$.

By Lemma 5 the dimension of the vector space $\mathcal{D}(P)$ of local $P$-differentials is at most equal to $n$. Making use of the particular form of the canonical parameters $f_{i}(x)$, we can easily prove the following theorem:

THEOREM 6. In order that the dimension of the vector space $\mathcal{D}(P)$ of local $P$-differentials have its maximum value $n$, it is necessary and sufficient that the coördinates $\alpha_{1}, \alpha_{2}, \cdots, \alpha_{n}$ of $P$ be separable quantities over $\kappa$.

Proof. By Lemma 5 the dimension of $\mathcal{D}(P)$ is equal to $n$ if and only if the transformation (10) (with $P$ instead of $W$ ) is nonsingular. Since $\tau f_{1}, \tau f_{2}, \cdots, \tau f_{n}$ form an independent basis of $\mathcal{H}\left(P / S_{n}\right)$, it follows that $\mathcal{D}(P)$ has dimension $n$ if and only if the local $P$-differentials $d_{P} f_{1}, d_{P} f_{2}, \cdots, d_{P} f_{n}$ are independent vectors. These vectors are independent if and only if the determinant of their components, that is, the Jacobian determinant $\left|\partial\left(f_{1}, f_{2}, \cdots, f_{n}\right) / \partial\left(x_{1}, x_{2}, \cdots, x_{n}\right)\right|_{x=\alpha}$, is different from zero. Since $f_{i}(x)$ is 
independent of $x_{j}, j>i$, this Jacobian determinant is given by the following product:

$$
\prod_{i=1}^{n}\left(\partial f_{i} / \partial x_{i}\right)_{x=\alpha}
$$

The first factor $\left(\partial f_{1} / \partial x_{1}\right)_{x=\alpha}$ is not equal to 0 if and only if $\alpha_{1}$ is separable over $\kappa$. The second factor $\left(\partial f_{2} / \partial x_{2}\right)_{x_{1}-\alpha_{1}, x_{2}=\alpha_{2}}$ is not equal to 0 if and only if $\alpha_{2}$ is separable over $\kappa\left(\alpha_{1}\right)$. Hence both factors are not equal to 0 if and only if $\alpha_{1}$ and $\alpha_{2}$ are separable over $\kappa$. In the same fashion it follows that the separability of all the coördinates $\alpha_{1}, \alpha_{2}, \cdots, \alpha_{n}$ is necessary and sufficient for the nonvanishing of the Jacobian determinant, q.e.d.

As an immediate corollary of Theorem 6, we obtain the following:

CRITERION FOR UNIFORMIZING PARAMETERs. Let the coördinates $\alpha_{1}, \alpha_{2}$, $\cdots, \alpha_{n}$ of $P$ be separable over $\kappa$ and let $u_{1}, u_{2}, \cdots, u_{n}$ be elements of $m\left(P / S_{n}\right)$. $A$ necessary and sufficient condition that the $u^{\prime}$ s be uniformizing parameters of $P$ is that the Jacobian determinant $\left|\partial\left(u_{1}, u_{2}, \cdots, u_{n}\right) / \partial\left(x_{1}, x_{2}, \cdots, x_{n}\right)\right|_{x=\alpha}$ be different from zero.

To see this it is only necessary to observe that if the $\alpha$ 's are separable, then by the preceding theorem and by Lemma 5 of the preceding section the linear transformation (10) (with $P$ instead of $W$ ) is nonsingular.

We add the obvious remark that if for a given set of elements $u_{1}, u_{2}, \cdots, u_{n}$ the above Jacobian determinant is different from 0 , then the differentials $d_{P} u_{i}$ are independent vectors of $\mathcal{D}(P)$, this vector space has then dimension $n$, and therefore the $\alpha$ 's are separable (Theorem 6), and the $u$ 's are uniformizing parameters of $P$.

We shall use the notation $J(v ; x)$ for the Jacobian matrix of a set $v_{1}, v_{2}, \cdots, v_{\nu}$ of rational functions of $x_{1}, x_{2}, \cdots, x_{n}$.

In the sequel we shall have occasion to use the following lemma:

Lemma 6. Let $\mathfrak{A}$ be an ideal in $\kappa\left[x_{1}, x_{2}, \cdots, x_{n}\right]$ and let $\left(F_{1}(x), F_{2}(x)\right.$, $\left.\cdots, F_{\nu}(x)\right)$ be a basis of $\mathfrak{A}$. If $\left(\xi_{1}, \xi_{2}, \cdots, \xi_{n}\right)$ is a zero of $\mathfrak{A}$ in some extension field of $\kappa$ and if the Jacobian matrix $J(F ; x)$ has rank $n$ at $x=\xi$, then the $\xi$ 's are separable algebraic over $\kappa$, and $(\xi)$ is a simple zero of $\mathfrak{A}$. Moreover, $n$ of the polynomials $F_{i}(x)$ are uniformizing parameters of the point $P(\xi)$.

Proof. Let $V$ be the irreducible algebraic variety in $S_{n}$ having $(\xi)$ as general point. Since $J(F ; x)$ has rank $n$ on $V$, it has still rank $n$ at some point $P\left(\alpha_{1}, \alpha_{2}, \cdots, \alpha_{n}\right)$ of $V$. We may assume that $\mid J\left(F_{1}, F_{2}, \cdots, F_{n} ; x_{1}, x_{2}\right.$, $\left.\cdots, x_{n}\right)\left.\right|_{x=\alpha} \neq 0$. By the preceding remark, the $\alpha$ 's are separable over $\kappa$, and the polynomials $F_{1}(x), F_{2}(x), \cdots, F_{n}(x)$ are uniformizing parameters of $P$. This implies that $(\alpha)$ is an isolated zero of the ideal $\left(F_{1}(x), F_{2}(x), \cdots, F_{n}(x)\right)$, that is, it is not a specialization of a zero of a higher dimension. But since $(\alpha)$ is a specialization of $(\xi)$ and $(\xi)$ is a zero of $\mathfrak{A}$, it follows that necessarily 
$(\xi)=(\alpha)$. Moreover, since $F_{1}(x), F_{2}(x), \cdots, F_{n}(x)$ are uniformizing parameters of $P$, they form a local $P$-basis of $\mathfrak{A}$. Hence $(\xi)$ is a simple zero of $\mathfrak{A}$ (Definition 2, §3.4).

Corollary. If the matrix $J(F ; x)$ of Lemma 6 has rank $n-\rho$ at $x=\xi$, say if $J\left(F ; x_{\rho+1}, x_{\rho+2}, \cdots, x_{n}\right)$ has rank $n-\rho$ at $x=\xi(0 \leqq \rho \leqq n)$, then $\xi_{\rho+1}, \xi_{\rho+2}, \cdots, \xi_{n}$ are separable algebraic over the field $\kappa\left(\xi_{1}, \xi_{2}, \cdots, \xi_{\rho}\right)$ (and hence the dimension of the "point" ( $\xi$ ) is not greater than $\rho$ ).

7.2. Criterion for simple points. The classical criterion for simple points of an algebraic variety (in terms of Jacobian matrices) can now be readily derived.

We shall take a slightly more general point of view and shall derive a criterion for a given point $P$ to be a simple zero of a given ideal $\mathfrak{A}$ in $\kappa\left[x_{1}, x_{2}, \cdots, x_{n}\right]$. If $\mathfrak{A}$ happens to be the ideal $I(V)$ of a given variety $V$ (irreducible or not), then we get the criterion for $P$ to be a simple point of $V$.

Let $V=V(\mathfrak{A})$ be the zero manifold of $\mathfrak{A}$ and let $P(\alpha)$ be a point of an irreducible $r$-dimensional component $V_{1}$ of $V$. Let $\left(F_{1}(x), F_{2}(x), \cdots, F_{\nu}(x)\right)$ be a basis of $\mathfrak{A}$.

THEOREM 7 (CLASSICAL CRITERION FOR SIMPLE POINTS). In order that $P(\alpha)$ be a simple zero of the ideal $\mathfrak{A}$ it is sufficient that the Jacobian matrix $J(F ; x)$ be of rank $n-r$ at $x=\alpha$. If the coördinates $\alpha_{1}, \alpha_{2}, \cdots, \alpha_{n}$ are separable over $\kappa$, then this condition is also necessary.

Proof. By Theorem $2^{\prime}(\$ 3.4),(\alpha)$ is a simple zero of $\mathfrak{A}$ if and only if exactly $n-r$ of the vectors $\tau F_{i}(x)$ are independent. If $\alpha_{1}, \alpha_{2}, \cdots, \alpha_{n}$ are separable over $\kappa$, the linear transformation (10) (with $P$ instead of $W$ ) is nonsingular. Hence, in this separable case, $(\alpha)$ is a simple zero of $\mathfrak{A}$ if and only if exactly $n-r$ of the $\nu$ local $P$-differentials $d_{P} F_{i}(x)$ are linearly independent. Hence in the separable case the above condition on the Jacobian matrix $J(F ; x)$ is both necessary and sufficient.

In the general case, given that $J(F ; x)$ has rank $n-r$ at $x=\alpha$, it follows that $n-r$ of the local $P$-differentials $d_{P} F_{i}(x)$ are linearly independent. Since in the linear transformation (10) we have $\tau F_{i}(x) \rightarrow d_{P} F_{i}(x)$, it follows a fortiori that $n-r$ of. the vectors $\tau F_{i}(x)$ are independent. Hence, by Theorem 2', $(\alpha)$ is a simple zero of $\mathfrak{A}$. .

CoRollaRy. If $\kappa$ is of characteristic 0 or a perfect field of characteristic $p \neq 0$, then the criterion of Theorem 7 is both necessary and sufficient. in $S_{n}$.

8. Continuation of the separable case: generalization to higher varieties

8.1. Extension of the preceding results. The results of the preceding section can be easily generalized to the case in which instead of a point $P$ we are dealing with an arbitrary $\rho$-dimensional irreducible variety $W$ in $S_{n}$. Let 
$\left(\eta_{1}, \eta_{2}, \cdots, \eta_{n}\right)$ be the general point of $W$ and let $t_{1}, t_{2}, \cdots, t_{n-\rho}$ be a given set of uniformizing parameters of $W$. Since the vectors $\tau t_{1}, \tau t_{2}, \cdots, \tau t_{n-p}$ form an independent basis of the vector space $\mathcal{H}\left(W / S_{n}\right)$, it follows that the linear transformation (10) is nonsingular if and only if the Jacobian matrix $J(t ; x)$ has rank $n-\rho$ at $x=\eta$. If this condition is satisfied for the given set of uniformizing parameters $t_{i}$, then the matrix $J\left(t^{\prime} ; x\right)$ will be of rank $n-\rho$ at $x=\eta$ for any other set of uniformizing parameters $t_{1}^{\prime}, t_{2}^{\prime}, \cdots, t_{n-\rho}^{\prime}$. It is clear that in all cases the above rank condition for the matrix $J\left(t^{\prime} ; x\right)$ is sufficient in order that given $n-\rho$ elements $t_{i}^{\prime}$ of $\boldsymbol{m}\left(W / S_{n}\right)$ be uniformizing parameters of $W$.

By analogy with Theorem 6 of the preceding section, the condition that $J(t ; x)$ have rank $n-\rho$ at $x=\eta$ can be translated into the equivalent condition that the field $\mathcal{F}(W)\left(=\kappa\left(\eta_{1}, \eta_{2}, \cdots, \eta_{n}\right)\right)$ be separably generated over $\kappa$. The proof of this is as follows:

Suppose that $J(t ; x)$ is of rank $n-\rho$ at $x=\eta$. We can write $t_{i}=\phi_{i}(x) / g(x)$, where $\phi_{i}(x), g(x) \in \kappa\left[x_{1}, x_{2}, \cdots, x_{n}\right]$ and $g(\eta) \neq 0$. Then it is clear that also $J(\phi ; x)$ has rank $n-\rho$ at $x=\eta$. We may assume that

$$
\left|J\left(\phi_{1}, \phi_{2}, \cdots, \phi_{n-\rho} ; x_{\rho+1}, x_{\rho+2}, \cdots, x_{n}\right)\right|_{x=\eta} \neq 0 .
$$

We take the field $\kappa^{*}=\kappa\left(\eta_{1}, \eta_{2}, \cdots, \eta_{\rho}\right)$ as new ground field and we set $\phi_{i}^{*}=\phi_{i}\left(\eta_{1}, \eta_{2}, \cdots, \eta_{\rho}, x_{\rho+1}, \cdots, x_{n}\right)$. The $n-\rho$ polynomials $\phi_{i}^{*}$ in $\kappa^{*}\left[x_{\rho+1}\right.$, $\left.x_{\rho+2}, \cdots, x_{n}\right], i=1,2, \cdots, n-\rho$, have the common zero $\left(\eta_{\rho+1}, \eta_{\rho+2}, \cdots, \eta_{n}\right)$, and we have by (12) that $\left|J\left(\phi_{1}^{*}, \phi_{2}^{*}, \cdots, \phi_{n-\rho}^{*} ; x_{\rho+1}, x_{\rho+2}, \cdots, x_{n}\right)\right|_{x=\eta} \neq 0$. Hence by Lemma 6 (where $n$ and $\nu$ should be replaced by $n-\rho$ ) we can conclude that $\eta_{\rho+1}, \eta_{\rho+2}, \cdots, \eta_{n}$ are separable algebraic over $\kappa\left(\eta_{1}, \eta_{2}, \cdots, \eta_{\rho}\right)$. Since $\mathcal{F}(W)$ is of degree of transcendency $\rho$ over $\kappa$, it follows that $\left(\eta_{1}, \eta_{2}, \cdots, \eta_{\rho}\right)$ is a separating transcendence basis of $\mathcal{f}(W) / \kappa$.

Conversely, if $\left(\eta_{1}, \eta_{2}, \cdots, \eta_{\rho}\right)$ is a separating transcendence basis of $\mathcal{F}(W) / \kappa$, then the adjunction of $\eta_{1}, \eta_{2}, \cdots, \eta_{\rho}$ to $\kappa$ achieves a reduction of $W$ to a point in $S_{n-\rho}^{\kappa^{*}}$ having separable coördinates. Hence by Theorem 6 it follows that if $t_{1}, t_{2}, \cdots, t_{n-\rho}$ are uniformizing parameters of $W$ then the determinant $\left|J\left(t_{1}, t_{2}, \cdots, t_{n-p} ; x_{\rho+1}, x_{\rho+2}, \cdots, x_{n}\right)\right|$ is different from zero at $x=\eta$.

We have thus shown that if $t_{1}, t_{2}, \cdots, t_{n-\rho}$ are elements of $\mathbf{m}(W / V)$, then the condition that the determinant $\left|J\left(t_{1}, t_{2}, \cdots, t_{n-\rho} ; x_{\rho+1}, x_{\rho+2}, \cdots, x_{n}\right)\right|$ be different from zero at $x=\eta$ is equivalent to the condition that the set $\left(\eta_{1}, \eta_{2}, \cdots, \eta_{\rho}\right)$ be a separating transcendence basis of $\kappa\left(\eta_{1}, \eta_{2}, \cdots, \eta_{n}\right) / \kappa$ and that $t_{1}, t_{2}, \cdots, t_{n-p}$ be uniformizing parameters of $W$. Since by a theorem due to MacLane $[8$, Lemma 2, p. 380 or Theorem 15, p. 384] the set $\left(\eta_{1}, \eta_{2}, \cdots, \eta_{n}\right)$ always contains a separating transcendence basis of the field $\kappa\left(\eta_{1}, \eta_{2}, \cdots, \eta_{n}\right) / \kappa$ if this field is separably generated over $\kappa$, our assertion is proved.

8.2. A proof of the theorem of MacLane. It is of some interest that the 
italicized statement which we have just proved can be used in order to derive the quoted theorem of MacLane. We proceed as follows:

Let $\left\{\zeta_{1}, \zeta_{2}, \cdots, \zeta_{\rho}\right\}$ be a separating transcendence basis of $\kappa\left(\eta_{1}, \eta_{2}\right.$, $\left.\cdots, \eta_{n}\right) / \kappa$ and let

$$
\zeta_{j}=\psi_{j}(\eta) / \psi_{0}(\eta)
$$$$
j=1,2, \cdots, \rho .
$$

We consider in a linear space $S_{n+\rho}$ the irreducible $\rho$-dimensional variety $W^{\prime}$ defined by the general point $\left(\eta_{1}, \eta_{2}, \cdots, \eta_{n}, \zeta_{1}, \zeta_{2}, \cdots, \zeta_{\rho}\right)$ (note that $\mathfrak{F}(W)=\mathfrak{F}\left(W^{\prime}\right)$, whence $W$ and $W^{\prime}$ are birationally equivalent varieties). Let

$$
\phi_{1}(x), \phi_{2}(x), \cdots, \phi_{n-p}(x)
$$

be $n-\rho$ polynomials in $\kappa\left[x_{1}, x_{2}, \cdots, x_{n}\right]$ which are uniformizing parameters of $W$. Let moreover

$$
\phi_{n-\rho+j}(x)=x_{n+j} \psi_{0}(x)-\psi_{j}(x), \quad j=1,2, \cdots, \rho .
$$

The $n$ polynomials $\phi_{1}(x), \phi_{2}(x), \cdots, \phi_{n}(x)$ in $x_{1}, x_{2}, \cdots, x_{n+\rho}$ vanish on $W^{\prime}$, that is, they vanish for $x_{i}=\eta_{i}, i=1,2, \cdots, n$, and $x_{n+j}=\zeta_{i}, j=1,2, \cdots, \rho$. $W e$ assert that these polynomials are uniformizing parameters of $W^{\prime}$. To prove this we have to show the following: if $F\left(x_{i}, x_{n+j}\right) \in \kappa\left[x_{1}, x_{2}, \cdots, x_{n+\rho}\right]$ and $F\left(\eta_{i}, \zeta_{j}\right)=0$, then there exists a polynomial $A\left(x_{i}, x_{n+j}\right)$ such that $A\left(\eta_{i}, \zeta_{j}\right) \neq 0$ and $A \cdot F$ belongs to the ideal generated by $\phi_{1}(x), \phi_{2}(x), \cdots, \phi_{n}(x)$ in $\kappa\left[x_{1}, x_{2}, \cdots, x_{n+\rho}\right]$. In view of (14) we can write for an arbitrary polynomial $F\left(x_{i}, x_{n+j}\right)$ an identity of the form:

$$
\left[\psi_{0}(x)\right]^{\nu} \cdot F(x)=\sum_{j=1}^{p} B_{j}(x) \phi_{n-\rho+j}(x)+G\left(x_{1}, x_{2}, \cdots, x_{n}\right),
$$

where $B_{j}(x) \in \kappa\left[x_{1}, x_{2}, \cdots, x_{n+\rho}\right]$ and $\nu$ is a suitable integer. Now if $F\left(\eta_{i}, \zeta_{j}\right)$ $=0$ then also $G(\eta)=0$. Since $\phi_{1}(x), \phi_{2}(x), \cdots, \phi_{n-\rho}(x)$ are uniformizing parameters of $W$, there must exist a polynomial $h(x)=h\left(x_{1}, x_{2}, \cdots, x_{n}\right)$ such that $h(\eta) \neq 0$ and $h(x) G(x) \equiv 0\left(\phi_{1}(x), \quad \phi_{2}(x), \cdots, \phi_{n-\rho}(x)\right)$. Hence $\left[\psi_{0}(x)\right]^{\nu} h(x) F(x) \equiv 0\left(\phi_{1}(x), \phi_{2}(x), \cdots, \phi_{n}(x)\right)$, and this proves our assertion, since $\psi_{0}(\eta) \neq 0$ and $h(\eta) \neq 0$.

By hypothesis, the set $\left(\eta_{i}, \zeta_{j}\right)$ contains the separating transcendence basis $\left(\zeta_{1}, \zeta_{2}, \cdots, \zeta_{p}\right)$ of $\mathcal{F}\left(W^{\prime}\right)(=\mathcal{F}(W))$, and we have just proved that $\phi_{1}, \phi_{2}, \cdots, \phi_{n}$ are uniformizing parameters of $W^{\prime}$. Hence by the result proved above (applied to $W^{\prime}$ instead of to $\left.W\right)$, the determinant $\left|J\left(\phi_{1}, \phi_{2}, \cdots, \phi_{n} ; x_{1}, x_{2}, \cdots, x_{n}\right)\right|$ must be different from zero on $W^{\prime}$ (that is, for $x_{i}=\eta_{i}$ and $x_{n+j}=\zeta_{j}$ ). This implies that the matrix $J\left(\phi_{1}, \phi_{2}, \cdots, \phi_{n-\rho} ; x_{1}, x_{2}, \cdots, x_{n}\right)$ is of rank $n-\rho$ at $x=\eta$, and therefore, again by the same result proved above (and applied to $W$ itself), the set $\left(\eta_{1}, \eta_{2}, \cdots, \eta_{n}\right)$ must contain a separating transcendence basis of $\kappa\left(\eta_{1}, \eta_{2}, \cdots, \eta_{n}\right) / \kappa$.

8.3. The singular manifold of an algebraic variety. As an immediate consequence of the preceding results we find that if $\kappa$ is a field of characteristic zero 
or is a perfect field of characteristic $p \neq 0$, then the singular manifold of an irreducible algebraic variety $V$ in $S_{n}$ is a proper algebraic subvariety of $V$. For let $\left(\xi_{1}, \xi_{2}, \cdots, \xi_{n}\right)$ be the general point of $V$ and let $\left(F_{1}(x), F_{2}(x), \cdots, F_{\nu}(x)\right)$ be a basis of $\boldsymbol{p}(V)$. If $r$ is the dimension of $V$, then under our assumption concerning the field $\kappa$, the Jacobian matrix $(J(F ; x))_{x=\xi}$ (which can never be of rank greater than $n-r$ since $\mathcal{D}(V)$ is at most of dimension $n-r$; see Lemma 5 , $\S 6.2)$ must be exactly of rank $n-r$. Hence the points of $V$ where the above matrix is of rank less than $n-r$ form a proper algebraic subvariety of $V$, and by Theorem 7 this subvariety coincides with the singular manifold of $V$. The above result will be extended later on to arbitrary ground fields.

We observe that if we had assumed only that the field $\mathcal{f}(V)$ is separably generated over $\kappa$, then on the basis of Theorem 7 we could only assert the following: the singular points of $V$ belong to some proper algebraic subvariety of $V$. But we could not assert immediately that the singular manifold is algebraic, because Theorem 7 gives only a sufficient condition for simple points.

To conclude this section we shall state the analogue of Theorem 7 for the case in which instead of a point $P(\alpha)$ we are dealing with an irreducible variety $W$ in $S_{n}$ having $\left(\eta_{1}, \eta_{2}, \cdots, \eta_{n}\right)$ as general point. We assume, as in Theorem 7 , that $W$ lies on some $r$-dimensional irreducible component of the zero manifold $\mathcal{U}(\mathfrak{A})$ of tine given ideal $\mathfrak{A}$.

TheOREM $7 '$ (Classical CRITERION FOR SIMPle SUbVARIETIEs). In order that $\left(\eta_{1}, \eta_{2}, \cdots, \eta_{n}\right)$ be a simple zero of the ideal $\mathfrak{A}$ it is sufficient that the Jacobian matrix $J(F ; x)$ be of rank $n-r$ at $x=\eta$. If the field $\kappa\left(\eta_{1}, \eta_{2}, \cdots, \eta_{n}\right)$ is separably generated over $\kappa$ then the above condition is also necessary.

The proof is the same as that of Theorem 7.

We observe that if $\kappa$ is a field of characteristic zero or a perfect field of characteristic $p \neq 0$, then Theorem 3 ( $\$ 4.2)$ follows from Theorems 7 and $7^{\prime}$.

\section{The nonseparable case.}

9.1. The dimension of $\mathcal{D}(W)$ as a numerical character of the field $\mathcal{F}(W)$. We say that an irreducible variety $W$ in $S_{n}$ presents the nonseparable case if the field $\mathcal{f}(W)$ is not separably generated over $\kappa$. In particular, if $W$ is a point $P$, the nonseparable case is characterized by the condition that at least one of the coördinates of $P$ is inseparable over $\kappa$.

Whether we are dealing with the separable or the nonseparable case, it is always true that if $\left(\eta_{1}, \eta_{2}, \cdots, \eta_{n}\right)$ is the general point of $W$ and if $\left(u_{1}, u_{2}, \cdots, u_{v}\right)$ is a local $W$-basis of $\boldsymbol{p}(W)$, that is, a basis of $\boldsymbol{m}\left(W / S_{n}\right)$, the rank of the Jacobian matrix $J(u ; x)$ at $x=\eta$ is independent of the local basis, for this rank gives the dimension of the vector space $\mathcal{D}(W)$ of local $W$-differentials. We know that the rank is always less than or equal to $n-\rho$, where $\rho$ is the dimension of $W$, and that the equality sign holds if and only if $W$ presents the separable case. So at least in the separable case it is true that the difference $n-(n-\rho)$ between the dimension of the ambient space $S_{n}$ and the 
dimension of $\mathcal{D}(W)$ is an intrinsic character of the field $\mathcal{f}(W)$, namely it is equal to the degree of transcendency of $\mathcal{F}(W) / \kappa$. We shall now show that also in the nonseparable case, if we denote by $n-\sigma$ the dimension of $\mathcal{D}(W)(\sigma>\rho)$, then $\sigma$ is an intrinsic character of the field $\mathcal{F}(W)$.

THEOREM 8. If $\operatorname{dim} \mathcal{D}(W)=n-\sigma<n-\rho$, then $\mathcal{F}(W)$ is a $\sigma$-fold extension of $\kappa$ (that is, $\mathcal{F}(W)$ can be obtained by adjoining to $\kappa$ a suitable set of $\sigma$ elements of $\mathcal{F}(W))$ but is not a $(\sigma-1)$-fold extension of $\kappa^{(6)}$.

Proof. We select for uniformizing parameters of $W$ as set of polynomials

$$
\phi_{1}(x), \phi_{2}(x), \cdots, \phi_{n-p}(x) .
$$

We have, by hypothesis, that the Jacobian matrix $J(\phi ; x)$ has rank $n-\sigma$ at $x=\eta$. Let, say, $J\left(\phi ; x_{\sigma+1}, x_{\sigma+2}, \cdots, x_{n}\right)$ be of rank $n-\sigma$ at $x=\eta$. Then by the corollary to Lemma $6(\$ 7.1)$ the field $\mathcal{F}(W)$ is a separable algebraic extension of the field $\kappa\left(\eta_{1}, \eta_{2}, \cdots, \eta_{\sigma}\right)$. Without loss of generality we may assume that $\eta_{1}, \eta_{2}, \cdots, \eta_{\rho}$ are algebraically independent over $\kappa$. Since $\sigma>\rho$, the field $\mathcal{F}(W)$ is an algebraic extension of the field $\Delta_{1}=\kappa\left(\eta_{1}, \eta_{2}, \cdots, \eta_{\sigma-1}\right)$. Let $\Delta_{1}^{\prime}$ be the greatest subfield of $\mathcal{f}(W)$ which is separable algebraic over $\Delta_{1}$. Every element of $\mathcal{F}(W)$ is either in $\Delta_{1}^{\prime}$ or is purely inseparable over $\Delta_{1}^{\prime}$. A fortiori every element of $\mathcal{F}(W)$ is either in $\Delta_{1}^{\prime}\left(\eta_{\sigma}\right)$ or is purely inseparable over $\Delta_{1}^{\prime}\left(\eta_{\sigma}\right)$. From this we conclude that $\mathcal{f}(W)=\Delta_{1}^{\prime}\left(\eta_{\sigma}\right)$ since $\kappa\left(\eta_{1}, \eta_{2}, \cdots, \eta_{\sigma}\right) \subseteq \Delta_{1}^{\prime}\left(\eta_{\sigma}\right)$ and since therefore $\mathcal{F}(W)$ is a separable algebraic extension of $\Delta_{1}^{\prime}\left(\eta_{\sigma}\right)$. Now $\Delta_{1}^{\prime}$ as a separable algebraic extension of $\Delta_{1}$ is a simple extension of $\Delta_{1}: \Delta_{1}^{\prime}$ $=\Delta_{1}(\theta)$. Hence $\mathcal{f}(W)=\Delta_{1}\left(\theta, \eta_{\sigma}\right)$. Of the two elements $\theta$, $\eta_{\sigma}$ one, namely $\theta$, is separable algebraic over $\Delta_{1}$. Hence $\mathcal{F}(W)$ is a simple extension of $\Delta_{1}: \mathcal{F}(W)$ $=\Delta_{1}(\alpha)=\kappa\left(\eta_{1}, \eta_{2}, \cdots, \eta_{\sigma-1}, \alpha\right)$, that is, $\mathcal{F}(W)$ is a $\sigma$-fold extension of $\kappa$.

Now let $\left(\zeta_{1}, \zeta_{2}, \cdots, \zeta_{m}\right)$ be any set of generators of $\mathcal{f}(W)$ over $\kappa: \mathcal{F}(W)$ $=\kappa\left(\zeta_{1}, \zeta_{2}, \cdots, \zeta_{m}\right)$. To complete the proof of the theorem we have to show that

$$
m \geqq \sigma .
$$

Since $\mathcal{f}(W)=\kappa\left(\eta_{1}, \eta_{2}, \cdots, \eta_{n}\right)$, we can write

$$
\zeta_{j}=\psi_{j}(\eta) / \psi_{0}(\eta)
$$

$$
j=1,2, \cdots, m,
$$

where $\psi_{0}(x), \psi_{j}(x) \in \kappa\left[x_{1}, x_{2}, \cdots, x_{n}\right]$ and $\psi_{0}(\eta) \neq 0$. We denote by $W^{\prime}$ the algebraic variety in $S_{n+m}$ whose general point is $\left(\eta_{1}, \eta_{2}, \cdots, \eta_{n}, \zeta_{1}, \zeta_{2}, \cdots, \zeta_{m}\right)$. Let moreover $W_{1}$ be the algebraic variety of $S_{m}$ whose general point is $(\zeta)=\left(\zeta_{1}, \zeta_{2}, \cdots, \zeta_{m}\right)$. The three varieties $W, W_{1}, W^{\prime}$ are birationally equivalent, and $W^{\prime}$ is symmetrically related to $W$ and $W_{1}$, namely $W^{\prime}$ is the join of $W$ and $W_{1}$.

(6) The theorem is not true in the separable case. If $\sigma=\rho=$ degree of transcendency of $\mathcal{F}(W) / \kappa$, then $\mathcal{F}(W)$ can always be regarded as a $(\rho+1)$-fold extension of $\kappa$ (a pure transcendental $\rho$-fold extension, followed by a simple separable extension), but not always as a $\rho$-fold extension of $\kappa$. 
If we set

$$
\phi_{n-\rho+j}(x)=x_{n+j} \psi_{0}(x)-\psi_{j}(x), \quad j=1,2, \cdots, m,
$$

then it follows as in our proof of MacLane's theorem ( $\$ 8.2)$ that the $n-\rho$ polynomials (15) together with the $m$ polynomials $\phi_{n-\rho+j}$ just introduced form a set of uniformizing parameters of $W^{\prime}$ in $S_{n+m}$. The Jacobian matrix $J\left(\phi_{1}, \phi_{2}, \cdots, \phi_{n+m-p} ; x_{1}, x_{2}, \cdots, x_{n+m}\right)$ has the form

$$
\left\|\begin{array}{cc}
J\left(\phi_{1}, \phi_{2}, \cdots, \phi_{n-p} ; x_{1}, x_{2}, \cdots, x_{n}\right) & 0 \\
* & \psi_{0}(x) E_{m}
\end{array}\right\|,
$$

where $E_{m}$ is the $m$-rowed unit matrix. Since the matrix at the upper left corner has rank $n_{i}-\sigma$ at $x_{i}=\eta_{i}, i=1,2, \cdots, n$, it follows that the above $(n+m-\rho)$ rowed matrix has rank $n+m-\sigma$ at $x_{i}=\eta_{i}$ and $x_{n+j}=\zeta_{j}(j=1,2, \cdots, m)$. Therefore

$$
\operatorname{dim} \mathscr{D}\left(W^{\prime}\right)=n+m-\sigma .
$$

If $\operatorname{dim} \mathscr{D}\left(W_{1}\right)=m-\sigma_{1}$, then interchanging the roles of $W$ and $W_{1}$ we get

$$
\operatorname{dim} \mathscr{D}\left(W^{\prime}\right)=m+n-\sigma_{1} \text {. }
$$

Consequently $\sigma=\sigma_{1}$. Since $m-\sigma_{1} \geqq 0$ we have the inequality (16), and this completes the proof of the theorem.

9.2. Abstract differentiations over $\kappa^{p}$. We shall assume in this section that the ground field $\kappa$ is of characteristic $p \neq 0$. Our main purpose at this stage is to derive general criteria for uniformizing parameters and simple loci, valid whether we are dealing with the separable or the nonseparable case. First we recall a few well known facts about abstract differentiation. Given a field $K$, a differenitation in $\mathbf{K}$ is an operator $D$ in $\mathbf{K}$ with the following properties: (1) $D y \in K$ is defined and is single-valued for all elements $y$ in $K$; (2) if $y, z \in K$ then $D(y-z)=D y-D z$ and $D(y z)=y D z+z D y$. The set of all differentiations in $\mathrm{K}$ can be regarded as a vector space over $\mathrm{K}$, if we define: (1) $\left(D_{1}+D_{2}\right) y$ $=D_{1} y+D_{2} y$, (2) $(c D) y=c \cdot D y$ for any $c$ in $K$. The zero vector is the trivial differentiation $D_{0}: D_{0} y=0$ for all $y$ in $\mathrm{K}$.

If $\mathbf{P}$ is a subfield of $\mathbf{K}$, we say that a differentiation $D$ in $\mathbf{K}$ is over $\mathbf{P}$ if $D a=0$ for all $a$ in $\mathrm{P}$. The differentiations in $\mathrm{K}$ over $\mathrm{P}$ also form a vector space over $\mathbf{K}$ (and hence also over $\mathbf{P}$ ).

Suppose that $K$ is of characteristic $p \neq 0$ and that $K^{p} \subseteq P$. For any element $y$ of $\mathrm{K}$ it is true then that either $y \in \mathrm{P}$ or $y^{p}$ is the least power of $y$ which belongs to $P$. Let $Z=\left\{z_{i}\right\}$ be a minimal (finite or infinite) set of generators of $\mathbf{K} / \mathbf{P}(Z=a$ relatively $p$-independent basis of $\mathbf{K} / \mathbf{P}$; see MacLane $[8, \mathrm{p} .376])$. The minimality of $Z$ is equivalent to the property that if $\left\{z_{1}, z_{2}, \cdots, z_{*}\right\}$ is any finite set of $s$ elements in $Z$, then the relative degree $\left[\mathrm{P}\left(z_{1}, z_{2}, \cdots, z_{s}\right): \mathrm{P}\right]$ is $p^{\circ}$. This property in its turn is equivalent to the condition that each ele- 
ment $y$ of $\mathrm{K}$ can be written in one and only one form as a polynomial in the $z_{i}$, with coefficients in $\mathrm{P}$, of degree not greater than $p-1$ in each of the arguments $z_{i}$.

It is clear that an abstract differentiation $D$ over $\mathbf{P}$ is uniquely determined if the values $a_{i}=D z_{i}, z_{i} \in Z$, are known, for if $y=\phi\left(\left\{z_{i}\right\}\right)$ is any element of $K$, then $D y=\sum_{i}\left(\partial \phi / \partial z_{i}\right) \cdot a_{i}$, only a finite number of partial derivatives $\partial \phi / \partial z_{i}$ being different from zero. On the other hand, if the elements $a_{i}$ are preassigned in an arbitrary fashion, then the above expression of $D y$ defines a differentiation in $\mathrm{K}$ over $\mathrm{P}$. The only thing that ought to be verified is that $D$ is then single-valued, and for that it is sufficient to show that if $y=0$ then $D y=0$. Now if we have a relation $\phi\left(\left\{z_{i}\right\}\right)=0$ over $P$, then in view of the $p$-independence of the elements $z_{i}$ the polynomial $\phi\left(\left\{X_{i}\right\}\right)$ in the indeterminates $X_{i}$ must belong to the ideal generated in $\mathrm{P}\left[\left\{X_{i}\right\}\right]$ by the elements $X_{i}^{p}-c_{i}$, where $c_{i}$ is the element $z_{i}^{p}$ of $\mathrm{P}$. That implies that the partial derivatives $\partial \phi / \partial X_{i}$ also belong to that ideal, whence $\partial \phi / \partial z_{i}=0$.

In particular we have for each $z_{i}$ in $Z$ the differentiation $D_{i}=\partial / \partial z_{i}$ defined by the conditions $D_{i} z_{i}=1, D_{i} z_{j}=0$ if $j \neq i$. If the relative degree of $\mathrm{K} / \mathrm{P}$ is finite, these differentiations form a vector basis for the set of all differentiations in $\mathrm{K}$ over $\mathrm{P}$.

We now identify $\mathrm{K}$ with the field $\kappa\left(x_{1}, x_{2}, \cdots, x_{n}\right)$, where $x_{1}, x_{2}, \cdots, x_{n}$ are indeterminates, and $\mathrm{P}$ with the field $\kappa^{p}$. If $Z=\left\{z_{j}\right\}$ is a $p$-independent basis of $\kappa / \kappa^{p}$, then we consider the following differentiations in $\kappa\left(x_{1}, x_{2}, \cdots, x_{n}\right)$ over $\kappa^{p}$ :

$$
D_{i}=\partial / \partial x_{i}, \quad D_{j}^{*}=\partial / \partial z_{j}
$$

The differentiations $D_{i}$ are actually over $\kappa$, while the $D_{j}^{*}$ are extensions of differentiations in $\kappa / \kappa^{p}$.

Let $W$ be an irreducible algebraic variety in $S_{n}$, and let $\left(\eta_{1}, \eta_{2}, \cdots, \eta_{n}\right)$ be the general point of $W$. The differentiations (17) leave invariant the quotient ring $\mathfrak{D}=Q\left(W / S_{n}\right)$. For any element $y$ in $\mathfrak{o}$ we set

$$
D_{i}^{W} y=\left(D_{i} y\right)_{x=\eta}, \quad D_{j}^{* W} y=\left(D_{i}^{*} y\right)_{x=\eta} .
$$

The $D_{i}^{W}$ and $D_{j}^{* W}$ are operators $D^{W}$ satisfying the following conditions: (1) $D^{W}$ is a single-valued mapping of $\mathrm{D}$ into the field $\mathcal{f}(W)=\kappa\left(\eta_{1}, \eta_{2}, \cdots, \eta_{n}\right)$; (2) $D^{w}(y-z)=D^{w} y-D^{w} z$; (3) $D^{w}(y z)=\bar{y} D^{w} z+\bar{z} D^{w} y$, where $\bar{y}$ and $\bar{z}$ are the $W$-residues of $y$ and $z$; (4) if $c \in \kappa^{p}$ then $D^{W} c=0$.

Any operator $D^{W}$ satisfying the first three of the above 4 conditions may be called a local $W$-differentiation. Condition (4) signifies that the differentiation is over $\kappa^{p}$. The local $W$-differentiations over $\kappa^{p}$ form in an obvious fashion a vector space over $\mathcal{F}(W)$. We denote this vector space by $K_{(}(W)$.

9.3. A lemma on the canonical uniformizing parameters. Our object now is to prove an important auxiliary lemma. Let $P\left(\alpha_{1}, \alpha_{2}, \cdots, \alpha_{n}\right)$ be a point in $S_{n}$ and let $f_{1}\left(x_{1}\right), f_{2}\left(x_{1}, x_{2}\right), \cdots, f_{n}\left(x_{1}, x_{2}, \cdots, x_{n}\right)$ be the canonical uniformiz- 
ing parameters of $P$ (see $\$ 7.1$ ). Let $\kappa_{1}$ be any subfield of $\kappa$ which is a finite extension of $\kappa^{p}$ and which contains all the coefficients of the $n$ polynomials $f_{i}\left(x_{1}, x_{2}, \cdots, x_{i}\right)$. Let $\left(z_{1}, z_{2}, \cdots, z_{\nu}\right)$ be a $p$-independent basis of $\kappa_{1} / \kappa^{p}$. The partial derivatives $D_{j}^{*} f_{i}=\partial f_{i} / \partial z_{j}, i=1,2, \cdots, n ; j=1,2, \cdots, \nu$, are well defined, and it is indifferent whether $D_{j}^{*}$ is regarded as a differentiation in $\kappa_{1}\left(x_{1}, x_{2}, \cdots, x_{n}\right)$, or as a differentiation in $\kappa\left(x_{1}, x_{2}, \cdots, x_{n}\right)$ defined by choosing a $p$-independent basis $Z$ of $\kappa / \kappa^{p}$ containing the elements $z_{1}, z_{2}, \cdots, z_{\nu}$.

Lemma 7. If each polynomial $f_{i}(x), i=1,2, \cdots, n$, depends only on $x_{1}^{p}, x_{2}^{p}, \cdots, x_{1}^{p}$, then the Jacobian matrix $J(f ; z)=\partial\left(f_{1}, f_{2}, \cdots, f_{n}\right) / \partial\left(z_{1}, z_{2}\right.$, $\left.\cdots, z_{\nu}\right)$ has rank $n$ at $x=\alpha$.

Proof. We shall proceed by induction with respect to the dimension $n$ of the space $S_{n}$. For $n=1$ the lemma asserts that the derivatives $\partial f_{1} / \partial z_{j}$, $j=1,2, \cdots, \nu$, are not all zero at $x_{1}=\alpha_{1}$. Let us assume that this assertion is false. Our assumption is then that $x=\alpha_{1}$ is a root of the polynomial $\partial f_{1}\left(x_{1}\right) / \partial z_{j}, j=1,2, \cdots, \nu$. Since $f_{1}\left(x_{1}\right)$ is an irreducible monic polynomial over $\kappa$ which vanishes at $x_{1}=\alpha_{1}$, it follows $\partial f_{1}\left(x_{1}\right) / \partial z_{j}$ is identically zero, that is, the coefficients of $f_{1}$ behave as "constants" under each of the differentiations $\partial / \partial z_{j}$ in $\kappa_{1}$ over $\kappa^{p}$. Therefore their derivatives are zero under all differentiations in $\kappa_{1} / \kappa^{p}$, and consequently all the coefficients of $f_{1}\left(x_{1}\right)$ are in $\kappa^{p}$. This implies that $f_{1}\left(x_{1}\right)$, which by hypothesis is a polynomial in $x_{1}^{p}$, is the pth power of a polynomial in $\kappa\left[x_{1}\right]$, in contradiction with the irreducibility of $f_{1}\left(x_{1}\right)$.

We assume now that the lemma is true for linear spaces $S_{n-1}$. Setting $\bar{\kappa}=\kappa\left(\alpha_{1}\right)$ we apply the lemma to $S_{n-1}^{\bar{\kappa}}$ and to the point $\bar{P}\left(\alpha_{2}, \alpha_{3}, \cdots, \alpha_{n}\right)$ in this space. The canonical uniformizing parameters of $\bar{P}$ are the polynomials $\bar{\phi}_{i}\left(x_{2}, x_{3}, \cdots, x_{i}\right)=f_{i}\left(\alpha_{1}, x_{2}, x_{3}, \cdots, x_{i}\right), i=2,3, \cdots, n$, and these depend only on $x_{2}^{p}, x_{3}^{p}, \cdots, x_{n}^{p}$. As field analogous to $\kappa_{1}$ we take the following field $\bar{\kappa}_{1}$ :

$$
\bar{\kappa}_{1}=\bar{\kappa}^{p}\left(z_{1}, z_{2}, \cdots, z_{v}\right)=\kappa_{1}\left(\alpha_{1}^{p}\right) .
$$

This field $\bar{\kappa}_{1}$ satisfies all the necessary requirements: (1) it is a subfield of $\bar{\kappa}$; (2) it is a finite extension of $\bar{\kappa}^{p}$; (3) it contains all the coefficients of the polynomials $\bar{\phi}_{2}, \bar{\phi}_{3}, \cdots, \bar{\phi}_{n}$ since by hypothesis only the powers of $\alpha_{1}^{p}$ occur explicitly in $f_{i}\left(\alpha_{1}, x_{2}, \cdots, x_{i}\right)$. Thus all the conditions of the lemma are satisfied. However, this time the elements $z_{1}, z_{2}, \cdots, z_{\nu}$ are not relatively $p$-independent over $\bar{\kappa}^{p}$. While the relative degree $\left[\kappa_{1}: \kappa^{p}\right]$ was $p^{\nu}$, we shall now show that

$$
\left[\bar{\kappa}_{1}: \bar{\kappa}^{p}\right]=p^{\nu-1} \text {, }
$$

and consequently $\nu-1$ of the elements $z_{j}$ form a p-independent basis of $\bar{\kappa}_{1} / \bar{\kappa}^{p}$. To see this we observe that $\left[\bar{\kappa}_{1}: \kappa^{p}\right]=\left[\bar{\kappa}_{1}: \bar{\kappa}^{p}\right] \cdot\left[\bar{\kappa}^{p}: \kappa^{p}\right]=\left[\bar{\kappa}_{1}: \bar{\kappa}^{p}\right] \cdot[\bar{\kappa}: \kappa]$, and that on the other hand $\left[\bar{\kappa}_{1}: \kappa^{p}\right]=\left[\bar{\kappa}_{1}: \kappa_{1}\right] \cdot\left[\kappa_{1}: \kappa^{p}\right]$. Hence

$$
\left[\bar{\kappa}_{1}: \bar{\kappa}^{p}\right] \cdot[\bar{\kappa}: \kappa]=\left[\kappa_{1}: \kappa^{p}\right] \cdot\left[\bar{\kappa}_{1}: \kappa_{1}\right] .
$$

Since $\bar{\kappa}=\kappa\left(\alpha_{1}\right)$ and $\alpha_{1}$ is a root of the irreducible polynomial $f_{1}\left(x_{1}\right)$ in $\kappa\left[x_{1}\right]$, 
we have $[\bar{k}: k]=\nu_{1}=$ degree of $f_{1}\left(x_{1}\right)$. On the other hand, by hypothesis we have $f_{1}\left(x_{1}\right)=\phi_{1}\left(x_{1}^{p}\right), \phi_{1}$ irreducible over $\kappa$, and $X_{1}=\alpha_{1}^{p}$ is a root of $\phi_{1}\left(X_{1}\right)$. Then $\phi_{1}\left(X_{1}\right)$ is also irreducible over the subfield $\kappa_{1}$ of $\kappa$, and since $\bar{\kappa}_{1}=\kappa_{1}\left(\alpha_{1}^{p}\right)$ it follows that $\left[\bar{\kappa}_{1}: \kappa_{1}\right]=$ degree of $\phi_{1}\left(X_{1}\right)=\nu_{1} / p$. Hence $[\bar{\kappa}: \kappa]=p \cdot\left[\bar{\kappa}_{1}: \kappa_{1}\right]$, and therefore, by $(20),\left[\kappa_{1}: k^{p}\right]=p \cdot\left[\bar{\kappa}_{1}: \bar{\kappa}^{p}\right]$, as asserted in (19).

We may then assume that $\left(z_{2}, z_{3}, \cdots, z_{v}\right)$ is a $p$-independent basis of $\bar{\kappa}_{1} / \bar{\kappa}^{p}$. We shall denote differentiation in $\bar{\kappa}_{1}$ over $\bar{\kappa}^{p}$ by $\bar{\partial} / \partial z_{j}, j=2,3, \cdots, \nu$. The differentiation $\bar{\partial} / \partial z_{j}$, if applied to elements of the subfield $\kappa_{1}$ of $\bar{\kappa}_{1}$, is expressible in terms of the old derivatives $\partial / \partial z_{j}, j=1,2, \cdots, \nu$, by the usual rule of composite differentiation:

$$
\bar{\partial} / \partial z_{j}=\partial / \partial z_{j}+\partial / \partial z_{1} \cdot \bar{\partial} z_{1} / \partial z_{j}
$$$$
j=2,3, \cdots, \nu \text {. }
$$

Here $\bar{\partial} z_{1} / \partial z_{j}$ is to be computed by making use of the relation

$$
\left[\partial f_{1} / \partial z_{j}+\partial f_{1} / \partial z_{1} \cdot \bar{\partial} z_{1} / \partial z_{j}\right]_{x_{1}-\alpha_{1}}=0 .
$$

From (21) and (22) we conclude that the matrix $(J(f ; z))_{x=\alpha}$ is equivalent to the matrix

$$
\left\|\begin{array}{cc}
\partial f_{1} / \partial z_{1}, & O \\
* & \bar{J}(\bar{\phi} ; z)
\end{array}\right\|_{x=\alpha}
$$

where $\bar{J}(\bar{\phi} ; z)=\bar{\partial}\left(\bar{\phi}_{2}, \bar{\phi}_{3}, \cdots, \bar{\phi}_{n}\right) / \partial\left(z_{2}, z_{3}, \cdots, z_{v}\right)$.

By our induction, the matrix $\bar{J}(\phi ; z)$ is of rank $n-1$ at $x=\alpha$. By the case $n=1$ and by (22) we have $\partial f_{1} / \partial z_{1} \neq 0$ at $x_{1}=\alpha_{1}$. Hence $J(f ; z)$ is of rank $n$ at $x=\alpha$, and this completes the proof of the lemma.

9.4. The vector space $\mathscr{D}^{*}(W)$ of the mixed local $W$-differentials. We go back to the vectors space $K(W)$ of the local $W$-differentiations ( $\$ 9.2)$. For a given element $\omega$ in $D$ we have that $D^{W} \omega$ is a linear function of the variable element $D^{W}$ in $K(W)$. This function we call the mixed local $W$-differential of $\omega$, and we denote it by $d_{W}^{*} \omega$, or simply by $d^{*} \omega$. The following relations are obvious: $d^{*}\left(\omega_{1}-\omega_{2}\right)=d^{*} \omega_{1}-d^{*} \omega_{2} ; d^{*}\left(\omega_{1} \omega_{2}\right)=\bar{\omega}_{1} d^{*} \omega_{2}+\bar{\omega}_{2} d^{*} \omega_{1}$, where $\bar{\omega}_{1}$ and $\bar{\omega}_{2}$ are the $\mathfrak{m}$-residues of $\omega_{1}$ and $\omega_{2}$ ( $\mathfrak{m}=$ the maximal ideal in $\left.\mathfrak{D}\right)$.

We are primarily interested in the differentials of elements in $\mathfrak{m}$. For $\omega$ in $\mathfrak{D}$ and $u$ in $\mathfrak{m}$ we have $d^{*}(\omega u)=\bar{\omega} d^{*} u$, where $\bar{\omega}$ is the $\mathfrak{m}$-residue of $\omega$, since the $\mathfrak{m}$-residue of $u$ is zero. It follows that the product $\bar{\omega} d^{*} u$ is again a differential of an element of $\mathfrak{m}$. Therefore, for variable $u$ in $\mathfrak{m}$ the differentials $d^{*} u$ form a vector space over $\mathcal{F}(W)$. We shall call this vector space the space of mixed local $W$-differentials, and we shall denote it by $\mathscr{D}^{*}(W)$.

From $d^{*}\left(\omega_{1} \omega_{2}\right)=\bar{\omega}_{1} d^{*} \omega_{2}+\bar{\omega}_{2} d^{*} \omega_{1}$ follows that if $u \in \mathfrak{m}^{2}$ then $d^{*} u=0$. From this we conclude, as in the case of the ordinary local differentials ( $\$ 6.2)$ that the mapping

$$
\tau u \rightarrow d^{*} u
$$

is a linear transformation of the local vector space $\mathcal{H}\left(W / S_{n}\right)$ onto the vector 
space $\mathcal{D}^{*}(W)$ of mixed local differentials at $W$.

9.5. The nonsingular character of the linear transformation $\mathcal{H}\left(W / S_{n}\right)$ $\rightarrow \Phi^{*}(W)$. Our main result for ground fields $\kappa$ of characteristic $p \neq 0$ is the following theorem:

THEOREM 9. The linear transformation (23) of $\mathcal{X}\left(W / S_{n}\right)$ onto $\mathcal{D}^{*}(W)$ is nonsingular (in other words: $\mathscr{D}^{*}(W)$ is of dimension $n-\rho$, where $\rho=$ dimension of $W)$.

Proof. We shall first prove Theorem 9 in the case in which $W$ is a point $P(\alpha)$. Let $n-\sigma=\operatorname{dim} \mathscr{D}(P), \sigma \geqq 0$. Then for any set of uniformizing parameters $t_{1}, t_{2}, \cdots, t_{n}$ of $P$ the matrix $J(t ; x)$ is of rank $n-\sigma$ at $P$. We take a fixed set of uniformizing parameters $t_{i}$ and we label the coördinates $x_{i}$ in $S_{n}$ in such a fashion that $J\left(t_{1}, t_{2}, \cdots, t_{n} ; x_{\sigma+1}, x_{\sigma+2}, \cdots, x_{n}\right)$ be of rank $n-\sigma$ at $x=\alpha$. If $\tau_{1}, \tau_{2}, \cdots, \tau_{n}$ is any other set of uniformizing parameters, then the matrix $J\left(\tau_{1}, \tau_{2}, \cdots, \tau_{n} ; x_{\sigma+1}, x_{\sigma+2}, \cdots, x_{n}\right)$ will also be of rank $n-\sigma$ at $x=\alpha$, for the $\tau$ 's are linear forms in the $t$ 's with coefficients in $D=Q\left(P / S_{n}\right)$, and the determinant of these coefficients is not 0 at $x=\alpha$. In particular we have then that if $f_{i}\left(x_{1}, x_{2}, \cdots, x_{i}\right), i=1,2, \cdots, n$, are the canonical uniformizing parameters of $P$ (relative to the particular order in which we have labeled the coördinates $\left.x_{i}\right)$, then $J\left(f_{1}, f_{2}, \cdots, f_{n} ; x_{\sigma+1}, x_{\sigma+2}, \cdots, x_{n}\right)$ is of rank $n-\sigma$ at $x=\alpha$. Since $f_{1}, f_{2}, \cdots, f_{\sigma}$ are independent of $x_{\sigma+1}, x_{\sigma+2}, \cdots, x_{n}$, it follows that

$$
\left|J\left(f_{\sigma+1}, f_{\sigma+2}, \cdots, f_{n} ; x_{\sigma+1}, x_{\sigma+2}, \cdots, x_{n}\right)\right|_{x=\alpha} \neq 0 .
$$

We have

$$
\begin{aligned}
& J\left(f_{1}, f_{2}, \cdots, f_{n} ; x_{1}, x_{2}, \cdots, x_{n}\right) \\
& =\left\|\begin{array}{cc}
J\left(f_{1}, f_{2}, \cdots, f_{\sigma} ; x_{1}, x_{2}, \cdots, x_{\sigma}\right) \\
* & J\left(f_{\sigma+1}, f_{\sigma+2}, \cdots, f_{n} ; x_{\sigma+1}, x_{\sigma+2}, \cdots, x_{n}\right)
\end{array}\right\|,
\end{aligned}
$$

and this matrix must be of rank $n-\sigma$ at $x=\alpha$. Hence, by (24), it follows that $\left(J\left(f_{1}, f_{2}, \cdots, f_{\sigma} ; x_{1}, x_{2}, \cdots, x_{\sigma}\right)_{x=\alpha}\right.$ is the zero matrix, that is, we must have: $\partial f_{i} / \partial x_{1}=\partial f_{i} / \partial x_{2}=\cdots=\partial f_{i} / \partial x_{i}=0$, at $x=\alpha, i=1,2, \cdots, \sigma$. This implies, in view of the defining properties of the canonical uniformizing parameters ( $\$ 7.1$, especially properties $\mathrm{c}$ and $\mathrm{d}$ ), that for $i=1,2, \cdots, \sigma$ the polynomial $f_{i}$ is a polynomial in $x_{1}^{p}, x_{2}^{p}, \cdots, x_{i}^{p}$. These polynomials are canonical uniformizing parameters of the point $P_{1}\left(\alpha_{1}, \alpha_{2}, \cdots, \alpha_{\sigma}\right)$ in $S_{\sigma}$. We are therefore in position to apply the preceding Lemma 7 (with $n$ replaced by $\sigma$ ), and we may assert that the matrix $J\left(f_{1}, f_{2}, \cdots, f_{\sigma} ; z_{1}, z_{2}, \cdots, z_{v}\right)$ is of rank $\sigma$ at $x=\alpha$. Here $\left(z_{1}, z_{2}, \cdots, z_{v}\right)$ is a $p$-independent basis of $k_{1} / \kappa^{p}$, where $\kappa_{1}$ is any field between $\kappa^{p}$ and $\kappa$ which is a finite extension of $\kappa^{p}$ and which contains all the coefficients of $f_{1}, f_{2}, \cdots, f_{\sigma}$. If we take $\kappa_{1}$ large enough so as to include in this field also the coefficients of $f_{\sigma+1}, f_{\sigma+2}, \cdots, f_{n}$, we can 
introduce the mixed Jacobian matrix:

$$
J(f ; x, z)=J\left(f_{1}, f_{2}, \cdots, f_{n} ; x_{1}, x_{2}, \cdots, x_{n} ; z_{1}, z_{2}, \cdots, z_{v}\right) .
$$

By (24) and (25), and in view of the fact that $J\left(f_{1}, f_{2}, \cdots, f_{\sigma} ; z_{1}, z_{2}, \cdots, z_{\nu}\right)$ is of rank $\sigma$ at $x=\alpha$, we conclude that the matrix $J(f ; x, z)$ is of rank $n$ at $x=\alpha$. This implies that the $n$ mixed local $P$-differentials $d^{*} f_{h}$ are linearly independent vectors of $D^{*}(P)$, for $d^{*} f_{h}$ is, by definition, a linear function on $K(P)$ whose value at $D_{i}^{P}$ and at $D_{j}^{* P}$ is respectively $\left(\partial f_{h} / \partial x_{i}\right)_{x=\alpha}$ and $\left(\partial f_{h} / \partial z_{j}\right)_{x=\alpha}$ $(i, h=1,2, \cdots, n ; j=1,2, \cdots, \nu)$. Hence $\mathcal{D}^{*}(P)$ is of dimension $n$, and this completes the proof of Theorem 9 when $W$ is a point.

In the general case we carry out our usual reduction to the zero-dimensional case. However, we must exercise caution in the course of this reduction, for there is one element in our definition of local $W$-differentiation which depends on the given ground field $\kappa$ (and not only on the quotient ring of $W$ ): it is the requirement that $D^{W} c=0$ if $c \in \kappa^{p}$. If $\left(\eta_{1}, \eta_{2}, \cdots, \eta_{n}\right)$ is the general point of $W$ and if we take as our new ground field the field $\bar{\kappa}=\kappa\left(x_{1}, x_{2}, \cdots, x_{\rho}\right)$ (where we assume that $\eta_{1}, \eta_{2}, \cdots, \eta_{\rho}$ are algebraically independent over $\kappa$ and hence are identifiable with $\left.x_{1}, x_{2}, \cdots, x_{\rho}\right)$, we shall be dealing with the point $\bar{P}\left(\eta_{\rho+1}, \eta_{\rho+2}, \cdots, \eta_{n}\right)$ in $S_{n-\rho}^{\bar{\kappa}}$. The local $\bar{P}$-differentiations $D^{\bar{P}}$ in 0 $\left(\mathrm{o}=Q\left(W / S_{n}\right)=Q\left(\bar{P} / S_{n-\rho}^{\bar{\alpha}}\right)\right)$ are then the local $W$-differentiations $D^{W}$ which satisfy the stronger requirement: $D^{W} \omega=0$ for $\omega$ in $\bar{\kappa}^{p}$. Therefore the vector space $K(\bar{P})$ is a (proper) subspace of $K(W)$. The elements $d^{*} u$ of $\mathcal{D}^{*}(W)$ are the linear functions on $K(W)$ defined by the various elements $u$ in the maximal ideal $\mathrm{m}$ of $\mathfrak{o}$, while the elements $\bar{d}^{*} u$ of $\mathcal{D}^{*}(\bar{P})$ are the linear functions on $K(\bar{P})$ defined by the same elements $u$. Hence $\bar{d}^{*} u$ is the linear function on $K(\bar{P})$ induced by $d^{*} u$, and therefore $\mathcal{D}^{*}(\bar{P})$ is a projection of $\mathcal{D}^{*}(W)$. The main point that has to be brought out, and this will complete the proof of Theorem 9 , is the following: the two vector spaces $\mathcal{D}^{*}(W)$ and $\mathcal{D}^{*}(\bar{P})$ have the same dimension. We have only to show that if $\bar{d}^{*} u=0$ then also $d^{*} u=0$. By Theorem 9 , which we have already proved in the case of points, it follows that if $\bar{d}^{*} u=0$ then $\tau u=0$. But then $u$ is necessarily an element in $\mathrm{m}^{2}$, and that, of course, implies that also $d^{*} u$ is equal to zero. Since by Theorem 9 (applied again to the point $\bar{P}$ in $\left.S_{n-\rho}^{\bar{\kappa}}\right)$ the space $\mathcal{D}^{*}(\bar{P})$ has dimension $n-\rho$, the proof of Theorem 9 is now complete.

9.6. General Jacobian criteria for uniformizing parameters and for simple loci. From Theorem 9 we now can derive readily the desired consequences.

TheOREM 10 (CRITERION FOR UNIFORMIZING PARAMETERs). A necessary and sufficient condition that given elements $u_{1}, u_{2}, \cdots, u_{n-\rho}$ in $\mathbf{m}\left(W / S_{n}\right)$ be uniformizing parameters of $W$ is that the mixed local $W$-differentials $d^{*} u_{1}, d^{*} u_{2}$, $\cdots, d^{*} u_{n-\rho}$ be independent vectors of $\mathcal{D}^{*}(W)$; or equivalently: if $\kappa_{1}$ is the field obtained by adjoining to $\kappa^{p}$ all the coefficients of the rational functions $u_{i}$ and if $\left(z_{1}, z_{2}, \cdots, z_{v}\right)$ is a p-independent basis of $\kappa_{1} / \kappa^{p}$, then the matrix $J(u ; x, z)$ should be of rank $n-\rho$ on $W$. 
The proof is obvious.

Let $\mathfrak{A}$ be a given ideal in $\kappa\left[x_{1}, x_{2}, \cdots, x_{n}\right]$ and let us assume that $W$ lies on an $r$-dimensional irreducible component of the zero manifold $U(\mathfrak{A})$ of $\mathfrak{A}$. Let $\left(F_{1}(x), F_{2}(x), \cdots, F_{h}(x)\right)$ be a basis of the ideal $\mathfrak{A}$, and let $\left(z_{1}, z_{2}, \cdots, z_{\nu}\right)$ be a $p$-independent basis of $\kappa_{1} / \kappa^{p}$, where $\kappa_{1}$ is the field obtained by adjoining to $\kappa^{p}$ all the coefficients of the polynomials $F_{j}(x)$. Let moreover $(\eta)=$ $\left(\eta_{1}, \eta_{2}, \cdots, \eta_{n}\right)$ be the general point of $W$.

THEOREM 11 (CRITERION FOR SIMPLE ZEROS). In order that $(\eta)$ be a simple zero of the ideal $\mathfrak{A}$ it is necessary and sufficient that the mixed Jacobian matrix $J(F ; x, z)$ be of rank $n-r$ on $W$ (that is, for $x=\eta)$.

The proof is the same as that of Theorem 7 (\$7.2), with all references to separability to be omitted.

COROLlaRy. If $V$ is an irreducible algebraic $r$-dimensional variety in $S_{n}$ and if the ideal $\mathfrak{A}$ of Theorem 11 is identified with the prime ideal $p(V)$, then the singular manifold of $V$ consists of those points of $V$ at which the mixed Jacobian matrix $J(F ; x, z)$ has rank less than $n-r$. This manifold is a proper algebraic subvariety of $V$.

The last assertion in the corollary follows from the fact that, in view of Theorem 11 , the rank of $J(F ; x, z)$ on $V$ itself must be exactly $n-r$.

10. Absolutely simple loci.

10.1. Algebraic ground field extensions. Let $R=\kappa\left[x_{1}, x_{2}, \cdots, x_{n}\right]$ be a polynomial ring in $n$ indeterminates $x_{i}$, over the ground field $\kappa$, and let $\kappa^{\prime}$ be an algebraic extension of $\kappa$. We denote by $R^{\prime}$ the polynomial ring $\kappa^{\prime}\left[x_{1}, x_{2}, \cdots\right.$, $\left.x_{n}\right]$.

If $\mathfrak{A}$ is an ideal in $R$ and if $\omega^{\prime}$ is an element of the extended ideal $R^{\prime} \cdot \mathfrak{A}$, then $\omega^{\prime}$ can be expressed in the form $\omega^{\prime}=\omega_{1} u_{1}^{\prime}+\omega_{2} u_{2}^{\prime}+\cdots+\omega_{8} u_{s}^{\prime}$, where $\omega_{i} \in \mathfrak{A}, u_{1}^{\prime}=1$ and $u_{1}^{\prime}, u_{2}^{\prime}, \cdots, u_{s}^{\prime}$ are elements of $\kappa^{\prime}$ which are lineary independent over $\kappa$. From this it follows that if $\omega^{\prime} \in R^{\prime} \cdot \mathfrak{A} \cap R$, then necessarily $\omega^{\prime}=\omega_{1}$ and $\omega_{2}=\omega_{3}=\cdots=\omega_{8}=0$. Hence the relation

$$
R^{\prime} \cdot \mathfrak{A} \cap R=\mathfrak{A}
$$

holds for any ideal $\mathfrak{A}$ in $R$.

LeMma 8. If $v_{1}^{\prime}, v_{2}^{\prime}, \cdots, v_{g}^{\prime}$ are elements of $\kappa^{\prime}$ whcih are linearly independent over $\kappa$, then an element of $R^{\prime}$ which is of the form $\omega_{1} v_{1}^{\prime}+\omega_{2} v_{2}^{\prime}+\cdots+\omega_{0} v_{0}^{\prime}$, $\omega_{i} \in R$, can belong to $R^{\prime} \cdot \mathfrak{A}$ only if $\omega_{i} \in \mathfrak{A}, i=1,2, \cdots, g$.

Proof. Let the element $\sum_{i=1}^{o} \omega_{i} v_{i}^{\prime}$ be denoted by $\omega^{\prime}$ and let $\kappa_{1}$ denote the field $k\left(v_{1}^{\prime}, v_{2}^{\prime}, \cdots, v_{0}^{\prime}\right)$. The set $\left\{v_{i}^{\prime}\right\}$ can be extended to an independent basis $\left\{v_{1}^{\prime}, v_{2}^{\prime}, \cdots, v_{g}^{\prime}, v_{g+1}^{\prime}, v_{g+2}^{\prime}, \cdots, v_{h}^{\prime}\right\}$ of $\kappa_{1} / \kappa$. Let $R_{1}=\kappa_{1} R=\kappa_{1}\left[x_{1}\right.$, $\left.x_{2}, \cdots, x_{n}\right]$ and let $\mathfrak{A}_{1}=R_{1} \cdot \mathfrak{A}$. We have $R^{\prime} \cdot \mathfrak{A}=R^{\prime} \cdot \mathfrak{A}_{1}$ and $\omega^{\prime} \in R_{1}$. Hence $\omega^{\prime} \in R^{\prime} \cdot \mathfrak{A}_{1} \cap R_{1}$, and therefore, by $(26), \omega^{\prime} \in \mathfrak{A}_{1}$. Since $R_{1}=\sum_{b=1}^{h} R \cdot v_{i}^{\prime}, \mathfrak{A}_{1}$ 
$=\sum_{i=1}^{n} \mathfrak{A} \cdot v_{i}^{\prime}$ and since $\omega^{\prime} \in \mathfrak{A}_{1}$, we can write $\omega^{\prime}$ in the form $\omega^{\prime}=\sum_{i=1}^{n} a_{i} v_{i}^{\prime}$, where the $a_{i}$ are in $\mathfrak{A}$. Since the $h$ elements $v_{i}^{\prime}$ are also linearly independent over the field $\kappa\left(x_{1}, x_{2}, \cdots, x_{n}\right)$, it follows that we must have $a_{i}=\omega_{i}, i=1$, $2, \cdots, g$, and $a_{j}=0$ for $j=g+1, g+2, \cdots, h$. Hence the $\omega_{i}$ are in $\mathfrak{A}$, as asserted.

Lemma 9. If $\mathfrak{p}$ is a prime ideal in $R$ and if $\mathfrak{p}^{\prime}$ is a prime ideal of $R^{\prime} \cdot \mathfrak{p}$, then $\mathfrak{p}^{\prime} \cap R=\mathfrak{p}$.

Proof. It is only necessary to show that $\mathfrak{p}^{\prime} \cap R \subseteq \mathfrak{p}$. Let $\omega$ be an element of $\mathfrak{p}^{\prime} \cap R$. Since $\omega \in \mathfrak{p}^{\prime}$ we must have $R^{\prime} \cdot \mathfrak{p}: R^{\prime} \cdot \omega \neq R^{\prime} \cdot \mathfrak{p}$. Let $\omega^{\prime}$ be an element of $R^{\prime} \cdot \mathfrak{p}: R^{\prime} \cdot \omega$ which is not in $R^{\prime} \cdot \mathfrak{p}$ and let us write $\omega^{\prime}$ in the form $\omega^{\prime}=\sum_{i=1}^{b} \omega_{i} v_{i}^{\prime}$, where $\omega_{i} \in R, v_{i}^{\prime} \in \kappa^{\prime}, v_{1}^{\prime}=1$ and $v_{1}^{\prime}, v_{2}^{\prime}, \cdots, v_{\sigma}^{\prime}$ are linearly independent over $\kappa$. We have $\omega^{\prime} \omega=\sum_{i=1}^{0} \omega_{i} \omega v_{i}^{\prime} \in R^{\prime} \cdot \mathfrak{p}$, and hence, by Lemma $8, \omega_{i} \omega \in \mathfrak{p}$, $i=1,2, \cdots, g$. On the other hand not all the $g$ elements $\omega_{i}$ can belong to $\mathfrak{p}$, since $\omega^{\prime} \notin R^{\prime} \cdot \mathfrak{p}$. Hence $\omega \in \mathfrak{p}, \mathfrak{p}^{\prime} \cap R \subseteq \mathfrak{p}$, and this establishes the lemma.

THEOREM 12. a. If $\kappa^{\prime}$ is a separable extension of $\kappa$ then the extension $R^{\prime} \cdot p$ of any prime ideal $\mathfrak{p}$ in $R$ is the intersection of prime ideals in $R^{\prime}$.

b. If the quotient field of $R / p$ is separably generated over $\kappa$, then a holds also for inseparable extensions $\kappa^{\prime} / \kappa$.

c. Under the assumption made in $\mathrm{b}$, the ideal $R^{\prime} \cdot \mathfrak{p}$ is prime if $\kappa^{\prime}$ is a pure inseparable extension of $\kappa$.

Proof. In parts a and $\mathrm{b}$ of the theorem we have to show that if some power of an element $\omega^{\prime}$ in $R^{\prime}$ belongs to $R^{\prime} \cdot \mathfrak{p}$, then $\omega^{\prime}$ itself belongs to $R^{\prime} \cdot \mathfrak{p}$. Now if $\omega^{\prime p} \in R^{\prime} \cdot \mathfrak{p}$, then $\omega^{\prime p}=\sum_{i=1}^{s} \omega_{i} u_{i}^{\prime}, \omega_{i} \in \mathfrak{p}, u_{i}^{\prime} \in \kappa^{\prime}$, so that $\omega^{\prime p} \in R_{1} \cdot \mathfrak{p}$, where $R_{1}=\kappa_{1}\left[x_{1}, x_{2}, \cdots, x_{n}\right]$ and $\kappa_{1}=\kappa\left(u_{1}^{\prime}, u_{2}^{\prime}, \cdots, u_{s}^{\prime}\right)=$ a finite extension of $\kappa$. To prove our assertion it is sufficient to prove that $\omega^{\prime} \in R_{1} \cdot \mathfrak{p}$. Hence for the proof of the theorem it is permissible to assume that $\kappa^{\prime}$ is a finite extension of $\kappa$ (a similar argument covers part c of the theorem).

Let $u_{1}, u_{2}, \cdots, u_{v}$ be an independent basis of $\kappa^{\prime}$ over $\kappa$, whence $R^{\prime}=R \cdot u_{1}+R \cdot u_{2}+\cdots+R \cdot u_{v}$. By (26) we have $R^{\prime} \cdot \mathfrak{p} \cap R=\mathfrak{p}$, and hence the integral domain $\bar{R}=R / \mathfrak{p}$ is a subring of $R^{\prime} / R^{\prime} \cdot \mathfrak{p}$. Consequently we can write:

$$
R^{\prime} / R^{\prime} \cdot \mathfrak{p}=\bar{R} \cdot u_{1}+\bar{R} \cdot u_{2}+\cdots+\bar{R} u_{v}=\kappa^{\prime} \cdot \bar{R}
$$

By Lemma 8 , the elements $u_{1}, u_{2}, \cdots, u_{v}$ are linearly independent with respect to $\bar{R}$. By Lemma 9 no element of $\bar{R}$, different from zero, is a zero divisor in $R^{\prime} / R^{\prime} \cdot \mathfrak{p}$. Hence if $\bar{\Sigma}$ denotes the quotient field of $\bar{R}$, the ring $R^{\prime} / R^{\prime} \mathfrak{p}$ can be embedded in the hypercomplex system

$$
\kappa \frac{\prime}{\bar{\Sigma}}=\bar{\Sigma} \cdot u_{1}+\bar{\Sigma} \cdot u_{2}+\cdots+\bar{\Sigma} \cdot u_{\nu},
$$

which is an extension of the field $\dot{\kappa}^{\prime} / \kappa$ regarded as a hypercomplex system $\kappa_{k}^{\prime}=\kappa \cdot u_{1}+\kappa \cdot u_{2}+\cdots+\kappa \cdot u_{\nu}$. It is well known that if $\kappa^{\prime}$ is a separable extension of $\kappa$ or if $\bar{\Sigma}$ is separably generated over $\kappa$, then $\kappa \frac{f}{\Sigma}$ is semi-simple 
(van der Waerden [10, p. 174]). If $\kappa \frac{\prime}{2}$ is semi-simple then $R^{\prime} / R^{\prime} \cdot \mathfrak{p}$ (which is a subring of $\kappa \frac{\prime}{\Sigma}$ ) has no proper nilpotent elements. This establishes parts a and $\mathrm{b}$ of the theorem.

As to part $\mathrm{c}$ it is sufficient to consider the case $\kappa^{\prime}=\kappa\left(a^{1 / p}\right)$, where $a \in \kappa$ and $p \neq 0$ is the characteristic of $\kappa$. In this case we have by $(27): R^{\prime} / R^{\prime} \cdot p$ $=\bar{R}[z] / z^{p}-a$, and hence $\kappa \frac{1}{\Sigma}=\bar{\Sigma}[z] / z^{p}-a$, where $z$ is an indeterminate. Since $\bar{\Sigma}$ is separably generated over $\kappa, z^{p}-a$ remains irreducible over $\bar{\Sigma}$. Hence $\kappa \frac{1}{\Sigma}$ is a field and therefore $R^{\prime} / R^{\prime} \cdot \mathfrak{p}$ is an integral domain. This completes the proof of the theorem.

Remark. The ring $\bar{R}[z] / z^{p}-a$ is at any rate a primary ring. Hence if $R / \mathfrak{p}$ is not separably generated over $\kappa$, we can still assert that if $\kappa^{\prime}$ is purely inseparable over $\kappa$ then $R^{\prime} \cdot \mathfrak{p}$ is a primary ideal.

10.2. Jacobian criterion for absolutely simple loci. Let $V$ be an irreducible algebraic variety in $S_{n}$ and let $p=p(V)$ be the corresponding prime ideal. We have seen that with one exception ( $\kappa^{\prime}$ inseparable over $\kappa$ and $\mathcal{F}(V)$ is not separably generated over $\kappa)$ the ideal $R^{\prime} \cdot \mathfrak{p}$ is the intersection of prime ideals in $R^{\prime}$ :

$$
R^{\prime} \cdot \mathfrak{p}=\bigcap_{i=1}^{h} \mathfrak{p}_{i}^{\prime}
$$

Correspondingly, the variety $V / \kappa$ may become reducible over $\kappa^{\prime}$; it splits into $h$ irreducible varieties:

$$
V / \kappa \rightarrow V / \kappa^{\prime}=\bigcup_{i=1}^{h} V_{i}^{\prime} / \kappa^{\prime}
$$

where $V_{i}^{\prime}=\mathcal{V}\left(p_{i}^{\prime}\right)$.

The varieties $V_{i}^{\prime}$ are all of the same dimension as $V$, for $\mathfrak{p}_{i}^{\prime} \cap R=\mathfrak{p}$ and $R^{\prime}$ is integrally dependent over $R$. In the exceptional case noted above, we have $h=1$ but $R^{\prime} \cdot p$ is, in general, not prime and hence does not coincide with $\boldsymbol{p}\left(V^{\prime}\right)$.

If $W$ is an irreducible subvariety of $V$, let

$$
W / \kappa \rightarrow W / \kappa^{\prime}=\bigcup_{i=1}^{0} W_{i}^{\prime} / \kappa^{\prime}
$$

describe the splitting of $W / \kappa$ into irreducible varieties upon the field extension $\kappa \rightarrow \kappa^{\prime}$. Let $q=p(W), q_{j}^{\prime}=p\left(W_{j}^{\prime}\right)$. Since $R^{\prime}$ is integrally dependent on $R$, it is well known that any $\mathfrak{p}_{i}^{\prime}$ is contained in at least one $\mathfrak{q}_{j}^{\prime}[6$, Theorem 3; 4 , Theorem 3]. Moreover, since $R$ is integrally closed, it is also well known that any $\mathfrak{q}_{j}^{\prime}$ contains at least one $\mathfrak{p}_{i}^{\prime}[6$, Theorem $6 ; 4$, Theorem 5]. Hence, each $V_{i}^{\prime}$ contains at least one of the varieties $W_{i}^{\prime}$ and each $W_{j}^{\prime}$ belongs to at least one of the varieties $V_{i}^{\prime}$.

We shall say that $W$ splits into simple subvarieties (under the given 
ground field extension $\kappa \rightarrow \kappa^{\prime}$ ) if each $W_{j}^{\prime}$ is simple for any of the varieties $V_{i}^{\prime}$ which contain $W_{j}^{\prime}$.

Definition 3. An irreducible simple subvariety $W / \kappa$ of $V / \kappa$ is absolutely simple for $V / \kappa$ if it splits into simple subvarieties under any algebraic extension of the ground field $\kappa$, and if $\mathcal{F}(V)$ is separably generated over $\kappa$.

Let $\left(f_{1}(x), f_{2}(x), \cdots, f_{\nu}(x)\right)$ be a basis of the ideal $\mathfrak{p}=\boldsymbol{p}(V)$.

THEOREM 13. A necessary and sufficient condition that an irreducible subvariety $W$ of an irreducible $r$-dimensional variety $V$ in $S_{n}$ be absolutely simple for $V$ is that the Jacobian matrix $J(f ; x)$ be of rank $n-r$ on $W$.

Proof. Assume that $W$ is absolutely simple for $V$. We pass from $\kappa$ to the algebraically perfect field $\kappa^{\prime}$ determined by $\kappa$. By assumption, $\mathcal{f}(V)$ is separably generated over $\kappa$. Hence, by Theorem 12, part $\mathrm{c}$, the ideal $\mathfrak{p}$ remains prime in $R^{\prime}$, so that $V$ remains irreducible over $\kappa^{\prime}$ and $\left(f_{1}(x), f_{2}(x), \cdots, f_{\nu}(x)\right)$ remains a basis of the prime ideal of $V$ over $\kappa^{\prime}$. Also $W$ remains irreducible (see the remark at the end of the proof of Theorem 12). By assumption $W / \kappa^{\prime}$ is simple for $V / \kappa^{\prime}$, and since $\kappa^{\prime}$ is a perfect field, it follows $(\S 8.3)$ that $J(f ; x)$ must be of rank $n-r$ on $W$.

Conversely, assume that $J(f ; x)$ is of rank $n-r$ on $W$. The matrix $J(f ; x)$ is then a fortiori of rank $n-r$ on $V$, and hence $(\$ 8.1) \mathcal{F}(V)$ is separately generated over $\kappa$. Let $\kappa^{\prime}$ be an arbitrary algebraic extension of $\kappa$, and let (28) and (29) be the corresponding decompositions of $V$ and $W$ over $\kappa^{\prime}$. If a given $W_{j}^{\prime}$ belongs to a given $V_{i}^{\prime}$, then we have that $f_{\mu}(x)=0$ on $V_{i}^{\prime}, \mu=1,2, \cdots, \nu$, and that $J(f ; x)$ has rank $n-r$ on $W_{j}^{\prime}$. Consequently (Theorem $7^{\prime}, \S 8.3$ ) $W_{j}^{\prime}$ is simple for $V_{i}^{\prime}$.

COROLlaRY 1. If $W / \kappa$ is absolutely simple for $V / \kappa$, then for any algebraic extension $\kappa^{\prime}$ of the ground field $\kappa$ it is true that each of the irreducible varieties $W_{j}^{\prime} / \kappa^{\prime}$ into which $W / \kappa$ splits belongs to only one of the varieties $V_{i}^{\prime} / \kappa^{\prime}$ into which $V / \kappa$ splits. In other words: each $W_{j}^{\prime} / \kappa^{\prime}$ is simple for the composite variety $V / \kappa^{\prime}$ in (28).

For the composite variety $V / \kappa^{\prime}$ is the zero manifold of the ideal $R^{\prime} \cdot\left(f_{1}(x), f_{2}(x), \cdots, f_{v}(x)\right)$, and the fact that $J(f ; x)$ is of rank $n-r$ on each $W_{j}^{\prime}$ implies that each $W_{j}^{\prime}$ is simple for this composite variety (Theorem $\left.7^{\prime}, \S 8.3\right)\left({ }^{7}\right)$.

Corollary 2. If it is known that the condition " $\mathcal{F}(V)$ is separably generated over $k "$ is already satisfied, then either one of the following two conditions is suff-

${ }^{7}$ It is clear from the proof that when we say " $W_{j}^{\prime} / \kappa^{\prime}$ is simple for the composite variety $V / \kappa^{\prime \prime}$ we actually mean more precisely the following: the general point of $W_{j}^{\prime} / \kappa^{\prime}$ is a simple zero of the ideal $R^{\prime} \cdot \mathfrak{p}$, where $\mathfrak{p}$ is the prime ideal of $V / \kappa$. This condition can replace in Definition 3 the condition that $\mathcal{f}(V)$ be separably generated over $\kappa$. For if $\mathcal{f}(V)$ is not separably generated over $\kappa$ then for a suitable extension $\kappa^{\prime}$ of $\kappa$ (for instance, for $\kappa^{\prime}=$ the algebraic closure of $\kappa$ ) the ideal $R^{\prime} \cdot p$ will be primary and therefore nowhere locally prime. 
cient in order that $W$ be absolutely simple for $V$ :

(1) $W / \kappa$ remains simple under the extension $\kappa \rightarrow \kappa^{\prime}=$ the algebraically perfect field determined by $\kappa$.

(2) $W / \kappa$ remains simple under any finite purely inseparable extension of $\kappa\left({ }^{8}\right)$.

The sufficiency of condition (1) has already been established in the course of the proof of Theorem 13. The sufficiency of condition (2) can be seen as follows. Let $\kappa^{\prime}$ be the field obtained by adjoining to $\kappa$ the $p$ th roots of the coefficients of the polynomials $f_{1}(x), f_{2}(x), \cdots, f_{\nu}(x)$ of a basis of $\boldsymbol{p}(V)$. The varieties $V / \kappa^{\prime}$ and $W / \kappa^{\prime}$ remain irreducible (Theorem 12 , part c). To these varieties we apply Theorem $11(\$ 9.6), \kappa^{\prime}$ being our new ground field. Since the coefficients of the polynomials $f_{\mu}(x), \mu=1,2, \cdots, \nu$, already belong to $\boldsymbol{\kappa}^{\prime p}$ and since these polynomials still form a base for the ideal $\boldsymbol{p}\left(V / \boldsymbol{\kappa}^{\prime}\right)$ (Theorem 12, part $\mathrm{c}$ ), it follows that the parameters $z_{j}$ are missing and that the matrix $J(f ; x, z)$ coincides with the matrix $J(f ; x)$. If $W / \kappa^{\prime}$ is simple for $V / \kappa^{\prime}$, then it follows that $J(f ; x)$ has rank $n-r$ on $W$, and hence $W / \kappa$ is absolutely simple for $V / \kappa$.

Corollary 3. Any simple irreducible subvariety $W$ of $V$ such that $\mathcal{f}(W)$ is separably generated over $\kappa$ is absolutely simple for $V$.

This is an immediate consequence of Theorem $7^{\prime}(\$ 8.3)$ and Theorem 13.

11. Intrinsic characterizations of absolutely simple loci.

11.1. Differential characterization. Using Theorem 13 it is not difficult to show that the concept of an absolutely simple subvariety $W$ of $V$ belongs to the local geometry of $V$ at $W$; in other words: whether $W$ is or is not absolutely simple for $V$ depends entirely on the structure of the quotient ring $\mathrm{D}=Q(W / V)$. The easiest way to see this is to use local $W$-differentiations on the variety $V$. We mean by such a differentiation a mapping $D$ of the above quotient ring $D$ into the field $\mathcal{f}(W)$ such that the following conditions are satisfied: (1) $D c=0$ if $c \in \kappa$; (2) $D\left(\omega_{1}-\omega_{2}\right)=D \omega_{1}-D \omega_{2}$; (3) $D\left(\omega_{1} \omega_{2}\right)=\bar{\omega}_{1} D \omega_{2}$ $+\bar{\omega}_{1} D \omega_{2}$, where $\bar{\omega}$ denotes the $W$-residue of $\omega$. These differentiations form a vector space over $\mathcal{f}(W)$. To prove the local character of the concept of an absolutely simple subvariety, we shall prove in this section the following stronger result:

Lemma 10. If $\left(f_{1}(x), f_{2}(x), \cdots, f_{\nu}(x)\right)$ is a basis of the ideal $\boldsymbol{p}(V)$ and if the Jacobian matrix $J(f ; x)$ has rank $n-\sigma$ at $W$, then there exist on $V$ exactly $\sigma$ local $W$-differentiations which are linearly independent over $\mathcal{F}(W)$.

In view of this lemma, the number of linearly independent local $W$-differentiations on $V$ is always greater than or equal to $r, r=\operatorname{dim} V$, and (by Theorem 13) this number is equal to $r$ if and only if $W$ is absolutely simple for $V$.

( $\left.{ }^{8}\right)$ The proof of (2) exhibits one specific finite, purely inseparable extension of $\kappa$, such that $W / \kappa$ is absolutely simple if and only if it remains simple under that particular extension of $\kappa$. 
Proof of the lemma. Let $(\xi)=\left(\xi_{1}, \xi_{2}, \cdots, \xi_{n}\right)$ and $(\eta)=\left(\eta_{1}, \eta_{2}, \cdots, \eta_{n}\right)$ be respectively the general point of $V$ and the general point of $W$. If $\omega=A(\xi) / B(\xi)$ is an element of $\mathrm{o}$ (whence $B(\eta) \neq 0$ ) and if $D$ is a local $W$-differentiation on $V$, then

$$
[B(\eta)]^{2} \cdot D \omega=\sum_{i=1}^{n}\left[B(\eta) \partial A / \partial \eta_{i}-A(\eta) \partial B / \partial \eta_{i}\right] D \xi_{i} .
$$

Hence the values of the $n$ derivatives $D \xi_{i}$ determine the differentiation $D$ uniquely. However these derivatives cannot be arbitrary elements of the field $\mathcal{F}(W)$, for they must satisfy the $\nu$ relations:

$$
G_{j}=\sum_{i=1}^{n} \partial f_{j} / \partial \eta_{i} \cdot D \xi_{i}=0, \quad i=1,2, \cdots, \nu .
$$

On the other hand, these relations are sufficient for the existence of a corresponding differentiation $D$, for then $D \omega$ can be defined by (30) for any element $\omega$ in 0 , and the only point to check is the following: if $F(x)=F\left(x_{1}, x_{2}, \cdots, x_{n}\right)$ is a polynomial with coefficients in $\kappa$ such that $F(\xi)=0$ then

$$
\sum_{i=1}^{n} \partial F / \partial \eta_{i} \cdot D \xi_{i}=0
$$

But this is obvious since $F(x)$ can be expressed in the form $\sum_{j=1}^{\nu} A_{j}(x) f_{j}(x)$, whence

$$
\sum_{i=1}^{n} \partial F / \partial \eta_{i} \cdot D \xi_{i}=\sum_{j=1}^{\nu} A_{j}(\eta) G_{j}
$$

If $J(f ; x)$ has rank $n-\sigma$ at $W$, that is, for $x=\eta$, the equations (31) have exactly $\sigma$ independent solutions $\left(D^{\mu} \xi_{1}, D^{\mu} \xi_{2}, \cdots, D^{\mu} \xi_{n}\right), \mu=1,2, \cdots, \sigma$, and this completes the proof of the lemma.

11.2. Direct verification of the local character of the definition. The local character of the concept of absolutely simple loci can also be deduced directly from the definition of these loci (Definition 3), without the use of Theorem 13. We shall only indicate the steps of the proof; the details present no difficulty whatsoever.

It will be sufficient to consider only finite algebraic extensions $\kappa^{\prime}$ of $\kappa$. Let $\kappa^{\prime}=\kappa \cdot u_{1}+\kappa \cdot u_{2}+\cdots+\kappa \cdot u_{\theta}$, where $\left(u_{1}, u_{2}, \cdots, u_{\theta}\right)$ is an independent basis of $\kappa^{\prime} / \kappa$. We extend the domain of coefficients in the hypercomplex system $\sum_{i=1}^{0} \kappa \cdot u_{i}$ from $\kappa$ to the quotient ring $D(=Q(W / V))$, that is, we consider the ring

$$
\mathfrak{o}^{\prime}=\mathfrak{o} \cdot u_{1}+\mathfrak{v} \cdot u_{2}+\cdots+\mathfrak{o} \cdot u_{g} .
$$

We use the notation of $\$ 10.1$. If $R^{\prime} \cdot \mathfrak{p}=\bigcap_{i=1}^{h} p_{i}^{\prime}$, then $\mathfrak{v}^{\prime}$ is a direct sum of $h$ integral domains: 


$$
\mathfrak{o}^{\prime}=\mathfrak{o}_{1}^{\prime} \oplus \mathfrak{o}_{2}^{\prime} \oplus \cdots \oplus \mathfrak{o}_{h}^{\prime} .
$$

If $V_{i}^{\prime}$ contains $s_{i}$ of the varieties $W_{j}^{\prime}$, then $o_{i}^{\prime}$ is a semi-local ring having exactly $s_{i}$ maximal prime ideals, and the $s_{i}$ quotient rings of these prime ideals in $0_{i}^{\prime}$ coincide with the $s_{i}$ quotient rings $Q\left(W_{i}^{\prime} / V_{i}^{\prime}\right)\left(i\right.$ fixed, $\left.W_{j}^{\prime} \subseteq V_{i}^{\prime}\right)$. Thus everything is described in terms of the quotient ring 0 , so that it is entirely a local matter whether or not each $W_{i}^{\prime}$ is simple for its carrier $V_{\boldsymbol{i}}^{\prime}$.

One could also proceed as follows. Let $\Sigma=\mathcal{F}(V)$ and let $\Sigma^{\prime} / \kappa$ be a composite extension of $\Sigma / \kappa$ and $\kappa^{\prime} / \kappa$ (see Chevalley [1]). Let $D^{\prime}=\kappa^{\prime} D$ be the least subring of $\Sigma^{\prime} / \kappa$ which contains both $D$ and $\kappa^{\prime}$. Then $D^{\prime}$ is a local ring and is in fact isomorphic to a quotient ring $Q\left(W_{j}^{\prime} / V_{i}^{\prime}\right)\left(W_{i}^{\prime} \subseteq V_{i}^{\prime}\right)$. There is only a finite number of nonequivalent or non-isomorphic composite extensions of $\Sigma / \kappa$ and $\kappa^{\prime} / \kappa$, and in this fashion we get the quotient rings $Q\left(W_{j}^{\prime} / V_{i}^{\prime}\right)$ for all pairs of indices $i$ and $j$ such that $W_{j}^{\prime} \subseteq V_{i}^{\prime}$.

11.3. André Weil's criterion. Another intrinsic characterization of absolutely simple loci has been communicated to me by André Weil. Given a simple irreducible subvariety $W$ of $V$ and given $r$ elements $\omega_{1}, \omega_{2}, \cdots, \omega_{r}(r=\operatorname{dim} V)$ in the quotient ring $\mathrm{D}(=Q(W / V))$, we say that the $\omega$ 's are uniformizing coördinates of $W$ (on $V$ ) if the following two conditions are satisfied: (1) the ring $\kappa\left[\omega_{1}, \omega_{2}, \cdots, \omega_{r}\right]$ contains a set of uniformizing parameters of $W ;(2)$ if $\zeta_{1}, \zeta_{2}, \cdots, \zeta_{r}$ denote the $W$-residues of the $\omega$ 's then $\mathcal{f}(W)$ is an algebraic extension of the field $\kappa\left(\zeta_{1}, \zeta_{2}, \cdots, \zeta_{r}\right)\left({ }^{(9)}\right.$.

Any simple $W$ possesses uniformizing coördinates. For we can take, for instance, for $\omega_{1}, \omega_{2}, \cdots, \omega_{\rho}(\rho=\operatorname{dim} W)$ any set of $\rho$ elements in $p$ whose $W$-residues are algebraically independent over $\kappa$, and for $\omega_{\rho+1}, \omega_{\rho+2}, \cdots, \omega_{r}$ a set of $r-\rho$ uniformizing parameters of $W$. For later purpose we prove the following lemma:

LEMMA 11. Any $r$ uniformizing coördinates of $W$ are algebraically independent over $\kappa$.

Proof. If $\rho=\operatorname{dim} W$ we may assume, in view of condition (2), that $\zeta_{1}, \zeta_{2}, \cdots, \zeta_{\rho}$ are algebraically independent over $\kappa$. Let $t_{1}, t_{2}, \cdots, t_{r-\rho}$ be elements of $\kappa\left[\omega_{1}, \omega_{2}, \cdots, \omega_{r}\right]$ which are uniformizing parameters of $W$. We shall prove the lemma by showing that the $r$ elements $\omega_{1}, \omega_{2}, \cdots, \omega_{\rho}, t_{1}, t_{2}, \cdots, t_{r-p}$ of $\kappa\left[\omega_{1}, \omega_{2}, \cdots, \omega_{r}\right]$ are algebraically independent over $\kappa$. Let $F\left(X_{1}, X_{2}\right.$, $\cdots, X_{r}$ ) be a nonzero polynomial in $\kappa\left[X_{1}, X_{2}, \cdots, X_{r}\right]$. We show that $F\left(t_{1}, t_{2}, \cdots, t_{r-\rho}, \omega_{1}, \omega_{2}, \cdots, \omega_{\rho}\right) \neq 0$. We write $F(X)$ in the following form:

$$
F(X)=\sum_{i=s}^{\infty} F_{i}(X)
$$

where $F_{i}$ is homogeneous, of degree $i$, in $X_{1}, X_{2}, \cdots, X_{r-\rho}$ and $F_{s}(X) \neq 0$. We

$\left({ }^{\circ}\right)$ We find it convenient to include condition (2) in the definition of uniformizing coördinates. When $W$ is a point this condition is, of course, vacuous. 
have $F_{i}\left(t_{1}, t_{2}, \cdots, t_{r-\rho} ; \omega_{1}, \omega_{2}, \cdots, \omega_{\rho}\right) \in \mathfrak{m}^{i}$, where $\mathfrak{m}=\boldsymbol{m}(W / V)$. On the other hand, since $F_{s}(X) \neq 0$ and since $\zeta_{1}, \zeta_{2}, \cdots, \zeta_{\rho}$ are algebraically independent over $\kappa$, the form $F_{s}\left(X_{1}, X_{2}, \cdots, X_{r-p} ; \zeta_{1}, \zeta_{2}, \cdots, \zeta_{\rho}\right)$ (having coefficients in the residue field $\mathcal{F}(W)$ of $\mathfrak{o})$ is not zero. Since $\mathfrak{o}$ is a regular ring it follows $(\$ 5.1)$ that $F_{s}(t ; \omega) \notin \mathfrak{m}^{s+1}$. Consequently $F(t ; \omega) \neq 0$, q.e.d.

Weil's criterion can be stated as follows:

THEOREM 14. A necessary and sufficient condition that a simple subvariety $W$ of $V$ be absolutely simple is that there exist uniformizing coördinates $\omega_{1}, \omega_{2}, \cdots, \omega_{r}$ of $W$ such that the $W$-residue $\zeta_{1}, \zeta_{2}, \cdots, \zeta_{r}$ of the $\omega$ 's generate over $\kappa$ the entire field $\mathcal{F}(W)$ (that is, $\mathcal{F}(W)=\kappa\left(\zeta_{1}, \zeta_{2}, \cdots, \zeta_{r}\right)$ ).

Proof. We first show that the condition is sufficient. Let $(\xi)=\left(\xi_{1}, \xi_{2}\right.$, $\left.\cdots, \xi_{n}\right)$ and $(\eta)=\left(\eta_{1}, \eta_{2}, \cdots, \eta_{n}\right)$ be respectively the general point of $V$ and of $W$. We consider the variety $V^{\prime}$ in $S_{n+r}$ having $\left(\xi_{1}, \xi_{2}, \cdots, \xi_{n}\right.$, $\left.\omega_{1}, \omega_{2}, \cdots, \omega_{r}\right)$ as general point. This variety $V^{\prime}$ is birationally equivalent to $V$ and it carries a subvariety $W^{\prime}$ with general point $\left(\eta_{1}, \eta_{2}, \cdots, \eta_{n}\right.$, $\left.\zeta_{1}, \zeta_{2}, \cdots, \zeta_{r}\right)$. Since the $\omega$ 's belong to the quotient ring $Q(W / V)$, it follows that $Q(W / V)=Q\left(W^{\prime} / V^{\prime}\right)$ (that is, the birational correspondence between $V$ and $V^{\prime}$ is regular at $W$ ). Hence it will be sufficient to show that $W^{\prime}$ is absolutely simple for $V^{\prime}$. Now if we deal with $V^{\prime}$ and $W^{\prime}$ we have a particular situation whereby the $r$ uniformizing coördinates $\omega_{i}$ of $W^{\prime}$ are among the coördinates of the general point of $V^{\prime}$. Hence we may assume that this situation prevailed already in the case of $W$, that is, we assume that the $\omega$ 's are in the set $\left(\xi_{1}, \xi_{2}, \cdots, \xi_{n}\right)$, say $\omega_{i}=\xi_{i}, i=1,2, \cdots, r$.

By hypothesis, the ring $\kappa\left[\xi_{1}, \xi_{2}, \cdots, \xi_{r}\right]$ contains a set of uniformizing parameters of $W$, say $f_{j}\left(\xi_{1}, \xi_{2}, \cdots, \xi_{r}\right), j=1,2, \cdots, r-\rho$. Let $f_{r-\rho+\mu}\left(x_{1}, x_{2}, \cdots, x_{n}\right), \mu=1,2, \cdots, n-r$, be uniformizing parameters of $V$ in $S_{n}$. Then it follows from Lemma 2, corollary (\$2.3) that the $n-\rho$ polynomials $f_{j}\left(x_{1}, x_{2}, \cdots, x_{r}\right), f_{r-\rho+\mu}\left(x_{1}, x_{2}, \cdots, x_{n}\right)$ are uniformizing parameters of $W$ in $S_{n}$ (that is, of $W$ regarded as subvariety of $S_{n}$ ). By hypothesis, the field $\mathcal{F}(W)\left(=\kappa\left(\eta_{1}, \eta_{2}, \cdots, \eta_{n}\right)\right)$ coincides with the field $\kappa\left(\eta_{1}, \eta_{2}, \cdots, \eta_{r}\right)$. Actually, for the purposes of the proof, we need only a weaker hypothesis, namely that $\mathcal{F}(W)$ is a separable extension of $\kappa\left(\eta_{1}, \eta_{2}, \cdots, \eta_{r}\right)\left({ }^{10}\right)$. For then there exists for $\mu=1,2, \cdots, n-r$ a polynomial $F_{\mu}\left(x_{1}, x_{2}, \cdots, x_{r}, x_{r+\mu}\right)$ such that $F\left(\eta_{1}, \eta_{2}, \cdots, \eta_{r}, \eta_{r+\mu}\right)=0$ and $\left(\partial F_{\mu} / \partial x_{r+\mu}\right)_{x=\eta} \neq 0$. Therefore the Jacobian determinant $\left|J\left(F_{1}, F_{2}, \cdots, F_{n-r} ; x_{r+1}, x_{r+2}, \cdots, x_{n}\right)\right|$ is not 0 at $x=\eta$. Since the $n-\rho$ polynomials $f_{j}, f_{n-\rho+\mu}$ are uniformizing parameters of $W$, it follows that a fortiori the Jacobian matrix $J\left(f_{1}, f_{2}, \cdots, f_{n-p} ; x_{r+1}, x_{r+2}, \cdots, x_{n}\right)$ is of rank not less than $n-r$ at $x=\eta$. Since $f_{1}, f_{2}, \cdots, f_{r-\rho}$ are independent of $x_{r+1}, x_{r+2}, \cdots, x_{n}$, it follows that the determinant $\mid J\left(f_{r-\rho+1}, f_{r-\rho+2}, \cdots, f_{n-\rho}\right.$;

(10) Hence we can weaken the sufficiency condition and assert that $W$ is absolutely simple for $V$ if there exist uniformizing coördinates $\omega_{1}, \omega_{2}, \cdots, \omega_{r}$ of $W$ such that $\mathcal{F}(W)$ is a separable algebraic extension of the field $\kappa\left(\zeta_{1}, \zeta_{2}, \cdots, \zeta_{r}\right)$, where $\zeta_{i}=W$-residue of $\omega_{i}$. 
$\left.x_{r+1}, x_{r+2}, \cdots, x_{n}\right) \mid$ is not 0 at $x=\eta$. Since $f_{r-\rho+\mu}(x)$ is zero on $V(\mu=1,2$, $\cdots, n-r)$ we conclude by Theorem 13 that $W$ is absolutely simple for $V$.

We now prove that the condition is necessary. If $W$ is absolutely simple for $V$ then we may assume that $\left|J\left(f_{1}, f_{2}, \cdots, f_{n-r} ; x_{r+1}, x_{r+2}, \cdots, x_{n}\right)\right| \neq 0$ on $W$, where $f_{1}(x), f_{2}(x), \cdots, f_{n-r}(x)$ are suitable polynomials which vanish on $V$. In that case $\eta_{r+1}, \eta_{r+2}, \cdots, \eta_{n}$ are (separable) algebraic over $\kappa\left(\eta_{1}, \eta_{2}, \cdots, \eta_{r}\right)$ (Lemma 6, corollary, §7.1), and $\rho$ of the first $r \eta^{\prime}$ 's, say $\eta_{1}, \eta_{2}, \cdots, \eta_{\rho}$, are algebraically independent over $\kappa$. We can therefore make the identification $x_{i}=\eta_{i}, i=1,2, \cdots, \rho$, and carry out a reduction to the zero-dimensional case. Instead of $S_{n}, V$, and $W$ we shall then have an $S_{n-\rho}^{*}, V^{*}$, and $W^{*}$, where $\kappa^{*}=\kappa\left(x_{1}, x_{2}, \cdots, x_{\rho}\right), \operatorname{dim} V^{*} / \kappa^{*}=r-\rho$, and $\operatorname{dim} W^{*} / \kappa^{*}=0$. Moreover, the general points of $V^{*}$ and $W^{*}$ are respectively $\left(\xi_{\rho+1}, \xi_{\rho+2}, \cdots, \xi_{n}\right)$ and $\left(\eta_{\rho+1}, \eta_{\rho+2}, \cdots, \eta_{n}\right)$, while the coördinates in $S_{n-\rho}^{*}$ are $x_{\rho+1}, x_{\rho+2}, \cdots, x_{n}$. From the above inequality, $\left|J\left(f_{1}, f_{2}, \cdots, f_{n-r} ; x_{r+1}, x_{r+2}, \cdots, x_{n}\right)\right| \neq 0$ at $W$, follows that also $W^{*}$ is an absolutely simple point of $V^{*}$. If we assume that the theorem is true for points, there will exist $r-\rho$ elements $\omega_{\rho+1}, \omega_{\rho+2}, \cdots, \omega_{r}$ in the quotient ring $\mathfrak{D}\left(=Q(W / V)=Q\left(W^{*} / V^{*}\right)\right)$ such that $\kappa\left[\omega_{\rho+1}, \omega_{\rho+2}, \cdots, \omega_{r}\right]$ contains a set of uniformizing parameters of $W^{*}$ (on $V^{*}$ ) and such that the $W^{*}$-residues of $\omega_{\rho+1}, \omega_{\rho+2}, \cdots, \omega_{r}$ generate over $\kappa^{*}$ the entire field $\mathcal{F}\left(W^{*}\right)$ $(=\mathcal{F}(W))$. If we set $\omega_{i}=\xi_{i}, i=1,2, \cdots, \rho$, the $r$ elements $\omega_{1}, \omega_{2}, \cdots, \omega_{r}$ will satisfy all the conditions of the theorem. Hence it is sufficient to prove the theorem for points of $V$.

We assume therefore that a given point $P\left(\alpha_{1}, \alpha_{2}, \cdots, \alpha_{n}\right)$ of $V$ is absolutely simple for $V$. Let, say,

$$
\left|J\left(f_{r+1}, f_{r+2}, \cdots, f_{n} ; x_{r+1}, x_{r+2}, \cdots, x_{n}\right)\right| \neq 0 \text { at } P,
$$

where $f_{r+1}, f_{r+2}, \cdots, f_{n}$ are suitable polynomials which vanish on $V$. Let $f_{i}\left(x_{1}, x_{2}, \cdots, x_{i}\right), i=1,2, \cdots, r$, be the canonical uniformizing parameters of the point $\left(\alpha_{1}, \alpha_{2}, \cdots, \alpha_{r}\right)$ in $S_{r}$. From (32) it follows that the $n-r$ polynomials $f_{r+j}\left(\alpha_{1}, \alpha_{2}, \cdots, \alpha_{r}, x_{r+1}, \cdots, x_{n}\right)$ are uniformizing parameters of the point $\left(\alpha_{r+1}, \alpha_{r+2}, \cdots, \alpha_{n}\right)$ in $S_{n-r}^{\kappa^{*}}$, where $\kappa^{*}=\kappa\left(\alpha_{1}, \alpha_{2}, \cdots, \alpha_{r}\right)$. From this one concludes by a simple calculation that the $n$ polynomials $f_{1}(x), f_{2}(x)$, $\cdots, f_{n}(x)$ are uniformizing parameters of the point $P$ in $S_{n}$. But since the last $n-r$ polynomials vanish on $V$, it follows that $f_{1}\left(\xi_{1}\right), f_{2}\left(\xi_{1}, \xi_{2}\right), \cdots$, $f_{r}\left(\xi_{1}, \xi_{2}, \ldots, \xi_{r}\right)$ are uniformizing parameters of the point $P$ on $V$. We have thus shown that $\xi_{1}, \xi_{2}, \cdots, \xi_{r}$ are uniformizing coördinates of $P$ on $V$.

Again by (32), we have that the field $\mathcal{f}(P)\left(=\kappa\left(\alpha_{1}, \alpha_{2}, \cdots, \alpha_{n}\right)\right)$ is a separable extension of $\kappa\left(\alpha_{1}, \alpha_{2}, \cdots, \alpha_{r}\right)$. From this it follows as in the proof of Theorem $8(\$ 9.1)$ that $\mathcal{F}(P)$ is a simple extension of $\kappa\left(\alpha_{1}, \alpha_{2}, \cdots, \alpha_{r-1}\right)$. We set

$$
\Delta_{1}=\kappa\left(\alpha_{1}, \alpha_{2}, \cdots, \alpha_{r-1}\right), \quad \Delta=\mathcal{F}(P)=\Delta_{1}\left(\alpha_{0}\right) .
$$

We proceed to show the existence of an element $\xi_{0}$ in $Q(P / V)$. whose $P$-residue 
is $\alpha_{0}$ and such that $\xi_{0}, \xi_{1}, \cdots, \xi_{r-1}$ are uniformizing coördinates of the point $P$ on $V$. This will complete the proof of the theorem.

We consider two cases, according as $\alpha_{r}$ is or is not separable over $\Delta_{1}$.

First case: $\alpha_{r}$ separable over $\Delta_{1}$. In this case also $\alpha_{0}$ is separable over $\Delta_{1}$, since $\alpha_{0}$ is separable over $\Delta_{1}\left(\alpha_{r}\right)$. Let $\phi\left(z ; \alpha_{1}, \alpha_{2}, \cdots, \alpha_{r-1}\right)$ be the monic irreducible polynomial in $\Delta_{1}[z]$ having $z=\alpha_{0}$ as a root. We may assume that $\phi$ is a polynomial in $\alpha_{1}, \alpha_{2}, \cdots, \alpha_{r-1}$ and $z$, with coefficients in $k$. Moreover, we have

$$
\phi_{\alpha_{0}}^{\prime}\left(\alpha_{0}, \alpha_{1}, \cdots, \alpha_{r-1}\right) \neq 0 .
$$

Let $\zeta_{0}$ be a fixed element in $Q(P / V)$ whose $P$-residue is $\alpha_{0}$, and let $\pi$ be a variable element of $\boldsymbol{m}(P / V)$. We set:

$$
\begin{aligned}
\xi_{0} & =\zeta_{0}+\pi, \\
\tau_{0} & =\phi\left(\zeta_{0}, \xi_{1}, \xi_{2}, \cdots, \xi_{r-1}\right), \\
t_{0} & =\phi\left(\xi_{0}, \xi_{1}, \xi_{2}, \cdots, \xi_{r-1}\right), \\
t_{i} & =f_{i}\left(\xi_{1}, \xi_{2}, \cdots, \xi_{i}\right),
\end{aligned}
$$$$
i=1,2, \cdots, r \text {, }
$$

so that $\alpha_{0}$ is also the $P$-residue of $\xi_{0}$, and $\tau_{0}, t_{0} \in \mathfrak{m}$, where $\mathfrak{m}=\boldsymbol{m}(P / V)$. We have

$$
t_{0} \equiv \tau_{0}+\partial \phi\left(\zeta_{0}, \xi_{1}, \cdots, \xi_{r-1}\right) / \partial \zeta_{0} \cdot \pi\left(\bmod \mathfrak{m}^{2}\right) .
$$

By (33) the partial derivative $\partial \phi / \partial \zeta_{0}$ in (34) does not belong to $m$ and is therefore a unit in $Q(P / V)$. We can therefore select $\pi$ in such a fashion as to have $\tau_{0}+\partial \phi / \partial \zeta_{0} \cdot \pi=t_{r}$. For such a choice of $\pi$ we shall have $t_{0} \equiv t_{r}\left(\bmod \mathrm{m}^{2}\right)$, and consequently $t_{1}, t_{2}, \cdots, t_{r-1}, t_{0}$ are uniformizing parameters of $P$ on $V$. From the above definition of the $t_{i}$ 's, $i=0,1, \cdots, r-1$, it follows then that $\xi_{0}, \xi_{1}, \cdots, \xi_{r-1}$ are uniformizing coördinates of $P$ on $V$.

Second case: $\alpha_{r}$ inseparable over $\Delta_{1}$. In this case we have that $\partial f_{r} / \partial x_{r}$ vanishes at $x=\alpha$, whence

$$
\partial f_{r}\left(\xi_{1}, \xi_{2}, \cdots, \xi_{r}\right) / \partial \xi_{r} \in \mathfrak{m} .
$$

Since $\alpha_{r} \in k\left(\alpha_{0}, \alpha_{1}, \cdots, \alpha_{r-1}\right)$, we can write

$$
\alpha_{r}=g\left(\alpha_{0}, \alpha_{1}, \cdots, \alpha_{r-1}\right),
$$

where $g$ is a polynomial with coefficients in $\kappa$. We now take for $\xi_{0}$ an arbitrary element of $Q(P / V)$ whose $P$-residue is $\alpha_{0}$. Let

$$
f_{0}\left(x_{0}, x_{1}, \cdots, x_{r-1}\right)=f_{r}\left(x_{1}, x_{2}, \cdots, x_{r-1}, g\left(x_{0}, x_{1}, \cdots, x_{r-1}\right)\right)
$$

and let $t_{0}=f_{0}\left(\xi_{0}, \xi_{1}, \cdots, \xi_{r-1}\right)$. We have

$$
\begin{aligned}
t_{0} \equiv & f_{r}\left(\xi_{1}, \xi_{2}, \cdots, \xi_{r-1}, \xi_{r}\right) \\
& +\partial f_{r}\left(\xi_{1}, \xi_{2}, \cdots, \xi_{r}\right) / \partial \xi_{r} \cdot\left[g\left(\xi_{0}, \xi_{1}, \cdots, \xi_{r-1}\right)-\xi_{r}\right]\left(\bmod m^{2}\right),
\end{aligned}
$$


since, by (36), $g\left(\xi_{0}, \xi_{1}, \cdots, \xi_{r-1}\right)-\xi_{r} \in \mathfrak{m}$. For this same reason and in view of (35) we conclude that $t_{0} \equiv f_{r}\left(\xi_{1}, \xi_{2}, \cdots, \xi_{r}\right)\left(\bmod \mathfrak{m}^{2}\right)$, that is, $t_{0} \equiv t_{r}$ $\left(\bmod \mathrm{m}^{2}\right)$, and therefore, as in the preceding case, we find that $\xi_{0}, \xi_{1}, \cdots, \xi_{r-1}$ are uniformizing coördinates of $P$ on $V$.

11.4. A criterion of analytical equivalence. Closely connected with Theorem 14 is another characterization of absolutely simple loci which is very suggestive and which we proceed to derive. For that purpose we introduce first of all the concept of analytically equivalent varieties. Let $V$ and $V^{\prime}$ be two irreducible algebraic varieties over $\kappa$, and let $W$ and $W^{\prime}$ be irreducible subvarieties respectively of $V$ and of $V^{\prime}$. Nothing is said about the dimensions of the ambient linear spaces $S_{n}, S_{n^{\prime}}$ in which $V$ and $V^{\prime}$ are immersed; these dimensions may very well be distinct. A priori we do not even assume that the dimensions of $V$ and $V^{\prime}$, or of $W$ and $W^{\prime}$, are the same. Let $\mathrm{o}=Q(W / V), \mathrm{o}^{\prime}=Q\left(W^{\prime} / V^{\prime}\right) ; \mathfrak{m}=\mathbf{m}(W / V), \mathfrak{m}^{\prime}=\boldsymbol{m}\left(W^{\prime} / V^{\prime}\right)$. Let moreover $\mathrm{o}^{*}$ be the completion of $\mathrm{D}$ with respect to the powers of $\mathfrak{m}$, and let similarly $\mathrm{o}^{\prime *}$ be the completion of $\mathrm{o}^{\prime}$ with respect to the powers of $\mathrm{m}^{\prime}$.

DEFINITION 4. The varieties $V$ and $V^{\prime}$ are said to be analytically equivalent at (or in the neighborhood of) $W$ and $W^{\prime}$ respectively, if the rings $\mathrm{o}^{*}$ and $\mathrm{o}^{*}$ are $k$-isomorphic.

Let $r$ and $\rho$ be the dimensions of $V$ and $W$ respectively; similarly let $r^{\prime}$ and $\rho^{\prime}$ be the dimensions of $V^{\prime}$ and $W^{\prime}$. The dimensions of the local rings $D^{*}$ and $D^{\prime *}$ are then $r-\rho$ and $r^{\prime}-\rho^{\prime}$ respectively. Consequently, if $V$ and $V^{\prime}$ are analytically equivalent at $W$ and $W^{\prime}$, then we must have $r-\rho=r^{\prime}-\rho^{\prime}$. Moreover, the residue field of $D^{*}$ is $\mathcal{F}(W)$ and the residue field of $\mathrm{o}^{\prime *}$ is $\mathcal{F}\left(W^{\prime}\right)$. Consequently the assumption of analytical equivalence implies that the field $\mathcal{F}(W)$ and $\mathcal{F}\left(W^{\prime}\right)$ are $\kappa$-isomorphic, that is, $W$ and $W^{\prime}$ must be birationally equivalent. Hence $\rho=\rho^{\prime}$ and therefore also $r=r^{\prime}$. Thus analytically equivalent varieties $V$ and $V^{\prime}$ must have the same dimension (but they need not be birationally equivalent).

Having established the concept of analytical equivalence, we now can state the following theorem:

THEOREM 15. A necessary and sufficient condition that an irreducible subvariety $W$ of $V$ be absolutely simple for $V$ is that $V$ be, locally at $W$, analytically equivalent to the linear space $S_{r}$, where $r=\operatorname{dim} V$.

Proof. Assume that $W$ is absolutely simple for $V$, and let $\omega_{1}, \omega_{2}, \cdots, \omega_{r}$ be elements of the quotient ring $\mathrm{o}$ of $W(\mathrm{D}=Q(W / V))$ which have the properties stated in Theorem 14. Let $W^{\prime}$ denote the irreducible variety in $S_{r}$ whose general point is $\left(\zeta_{1}, \zeta_{2}, \cdots, \zeta_{r}\right)$, where $\zeta_{i}=W$-residue of $\omega_{i}$, and let $\mathfrak{o}^{\prime}=Q\left(W^{\prime} / S_{r}\right)$. As usual we denote by $\mathfrak{m}$ and $\mathfrak{m}^{\prime}$ the maximal ideals of $\mathfrak{o}$ and $D^{\prime}$ respectively.

By Lemma 11, the uniformizing coördinates $\omega_{1}, \omega_{2}, \cdots, \omega_{r}$ are algebraically independent over $\kappa$. Hence we can identify the ring $\kappa\left[\omega_{1}, \omega_{2}, \cdots, \omega_{r}\right]$ 
with the coördinate ring (the polynomial ring) $\kappa\left[x_{1}, x_{2}, \cdots, x_{r}\right]$ of $S_{r}$. The elements of $\mathfrak{o}^{\prime}$ are then of the form $\phi(\omega) / \psi(\omega), \psi(\zeta) \neq 0$. Hence:

(a) $\mathfrak{D}^{\prime}$ is a subring of $\mathfrak{D}$.

The ring $\mathfrak{o}$, topologized by the powers of $\mathfrak{m}$, shall be referred to as the $\mathfrak{m}$-adic ring $\mathfrak{D}$. We prove now that:

(b) The ring $\mathrm{o}^{\prime}$ is everywhere dense in the $\mathrm{m}$-adic ring $\mathrm{o}$.

We have to show that if $u$ is any element of $\mathfrak{o}$ and if $i$ is ar y positive integer, then there exists a rational function $\phi_{i}(\omega) / \psi_{i}(\omega)$ such that $\psi_{i}(\zeta) \neq 0$ and $u \equiv \phi_{i}(\omega) / \psi_{i}(\omega)\left(\bmod m^{i+1}\right)$. If $i=0$ we make use of the fact that $\mathcal{f}(W)$ $=\kappa\left(\zeta_{1}, \zeta_{2}, \cdots, \zeta_{r}\right)$. Because of this we can write: $W$-residue of $u=\phi_{0}(\zeta) / \psi_{0}(\zeta)$, and hence $u=\phi_{0}(\omega) / \psi_{0}(\omega)$ ( $\left.\bmod \mathfrak{m}\right)$. Assuming that $\phi_{i}(\omega) / \psi_{i}(\omega)$ has already been shown to exist for all $i<s$, we make use of the fact that the $\omega$ 's are uniformizing coördinates. Let $t_{1}, t_{2}, \cdots, t_{r-\rho}$ be elements of $\kappa\left[\omega_{1}, \omega_{2}, \cdots, \omega_{r}\right]$ which are uniformizing parameters of $W$. We have $u-\phi_{s-1}(\omega) / \psi_{s-1}(\omega) \in \mathfrak{m}^{s}$, hence we can write $u-\phi_{s-1}(\omega) / \psi_{s-1}(\omega)$ as a form in $t_{1}, t_{2}, \cdots, t_{r-\rho}$, of degree $s$, with coefficients in $\boldsymbol{D}$. By the case $i=0$, each of these coefficients is congruent $\bmod \mathfrak{m}$ to elements of $\mathfrak{o}^{\prime}$. If these elements-rational functions in the $\omega^{\prime} \mathrm{s}$-are substituted for the coefficients, then we get a rational function $\alpha(\omega) / \beta(\omega)$ such that $\beta(\zeta) \neq 0$ and such that $u \equiv \phi_{s-1}(\omega) / \psi_{s-1}(\omega)+\alpha / \beta\left(\bmod m^{s+1}\right)$. This yields the desired rational function $\phi_{s}(\omega) / \psi_{s}(\omega)$ and establishes the assertion (b).

An element $\phi(\omega) / \psi(\omega)$ of $\mathfrak{o}^{\prime}$ belongs to $\mathrm{m}^{\prime}$ if and only if $\phi(\zeta)=0$, that is, if and only if $\phi(\omega) / \psi(\omega) \in \mathfrak{m}$. Hence

$$
\mathfrak{m} \cap 0=\mathfrak{m}^{\prime} .
$$

If $u \in \mathfrak{m}$ and if we write, by (b), $u \equiv \phi_{1}(\omega) / \psi_{1}(\omega)\left(\bmod \mathfrak{m}^{2}\right)$, then $\phi_{1}(\omega) / \psi_{1}(\omega)$ $\in \mathfrak{m} \cap \mathfrak{v}^{\prime}$, that is, $\phi_{1}(\omega) / \psi_{1}(\omega) \in \mathfrak{m}^{\prime}$, by (37). Consequently $\mathfrak{m}=\mathfrak{v} \cdot \mathfrak{m}^{\prime}+\mathfrak{m}^{2}$, and from this we conclude as in $\$ 2.1$ that

$$
\mathfrak{m}=\mathfrak{o} \cdot \mathfrak{m}^{\prime} .
$$

By (38), any basis of $\mathfrak{m}^{\prime}$ is also a basis of $\mathfrak{m}$. Since $W^{\prime}$ is of dimension $\rho$ and is immersed in a linear $S_{r}$, it follows that a minimal basis of $\mathfrak{m}^{\prime}$ consists exactly of $r-\rho$ elements. Let $\tau_{1}, \tau_{2}, \cdots, \tau_{r-\rho}$ be any minimal basis of $\mathfrak{m}^{\prime}$, that is, the $\tau$ 's are uniformizing parameters of $W^{\prime}$ in $S_{r}^{\prime}$. Then the $\tau$ 's form also a minimal base of $\mathrm{m}$, and therefore they are, as well as $t$ 's, uniformizing parameters of $W$ on $V$. Since the $t^{\prime}$ s are in $\mathfrak{m}^{\prime}$ we have relations of the form: $t_{i}=\sum_{j=1}^{r-\rho} \alpha_{i j} \tau_{j}, \alpha_{i j} \in \mathrm{O}^{\prime}, i=1,2, \cdots, r-\rho$. Since both the $t^{\prime}$ 's and the $\tau^{\prime}$ 's are uniformizing parameters of $W$ on $V$ and since the $\alpha_{i j}$ are also in $\mathfrak{D}$, it follows that the determinant $\left|\alpha_{i j}\right|$ is a unit in $\mathfrak{b}$, that is, it is not an element of $\mathrm{m}$. But since $\left|\alpha_{i j}\right| \in \mathfrak{o}^{\prime}$ it follows by (37) that $\left|\alpha_{i j}\right| \notin \mathfrak{m}^{\prime}$, and therefore $\left|\alpha_{i j}\right|$ is also a unit in $\mathfrak{o}^{\prime}$. Consequently $t_{1}, t_{2}, \cdots, t_{r-\rho}$ are also uniformizing parameters of $W^{\prime}$. What we have shown is that not only is every minimal basis of $\mathfrak{m}^{\prime}$ a minimal basis of $\mathfrak{m}$, but also that any minimal basis of $\mathfrak{m}$ whose elements belong to $\mathfrak{o}^{\prime}$ is necessarily a minimal basis of $\mathfrak{m}^{\prime}$. 
If $u$ is an element of $\mathfrak{m}^{\prime i}$ but is not in $\mathfrak{m}^{\prime i+1}$, then $u=\phi_{i}\left(t_{1}, t_{2}, \cdots, t_{r-\rho}\right)$, where $\phi_{i}$ is a form of degree $i$ with coefficients in $\mathfrak{o}^{\prime}$, but not all in $\mathfrak{m}^{\prime}$. Therefore these coefficients are not all in $\mathfrak{m}$, and consequently $u \in \mathfrak{m}^{i}, u \in \mathfrak{m}^{i+1}$. Hence

$$
\mathfrak{m}^{i} \cap \mathrm{o}^{\prime}=\mathfrak{m}^{\prime i}, \quad i=1,2, \cdots .
$$

The relations (38) and (39) imply that:

(c) The $\mathfrak{m}^{\prime}$-adic ring $\mathfrak{D}^{\prime}$ is a subspace of the $\mathfrak{m}$-adic ring $\mathfrak{D}$.

From (a), (b) and (c) we conclude that the complete rings $\mathfrak{D}^{*}$ and $\mathfrak{b}^{\prime *}$ coincide (see Chevalley [2, Theorem 1, p. 698]), and therefore $V$ and $S_{r}$ have analytically equivalent neighborhoods at $W$ and $W^{\prime}$ respectively.

Conversely, assume that there exists in $S_{r}$ a $\rho$-dimensional irreducible variety $W^{\prime}$ such that $V$ and $S_{r}$ are analytically equivalent in the neighborhoods of $W$ and $W^{\prime}$ respectively. Let $x_{1}, x_{2}, \cdots, x_{r}$ be variable coördinates in $S_{r}$, and let $\left(\zeta_{1}^{\prime}, \zeta_{2}^{\prime}, \cdots, \zeta_{r}^{\prime}\right)$ be the general point of $W^{\prime}$. Let $\mathfrak{o}^{\prime}=Q\left(W^{\prime} / S_{r}\right)$, $\mathfrak{m}^{\prime}=\boldsymbol{m}\left(W^{\prime} / S_{r}\right)$. We denote by $\mathfrak{o}^{*}$ and $\mathfrak{o}^{\prime *}$ the complete rings determined respectively by the $\mathfrak{m}$-adic ring $\mathfrak{D}$ and the $\mathfrak{m}^{\prime}$-adic ring $\mathfrak{o}^{\prime}$; and we denote by $\mathfrak{m}^{*}$ and $\mathfrak{m}^{\prime *}$ the maximal ideals in these rings. By hypothesis, $\mathfrak{o}^{\prime *}$ and $\mathfrak{o}^{*}$ are $\kappa$-isomorphic. Let $f$ denote a fixed $\kappa$-isomorphism of $\mathfrak{b}^{\prime *}$ onto $\mathrm{D}^{*}$.

We can find a minimal basis of $\mathfrak{m}^{\prime}$ consisting of polynomials in $\kappa\left[x_{1}, x_{2}, \cdots, x_{r}\right]$. Let $\left(t_{1}^{\prime}, t_{2}^{\prime}, \cdots, t_{r-\rho}^{\prime}\right), t_{t}^{\prime}=\phi_{i}\left(x_{1}, x_{2}, \cdots, x_{r}\right)$, be such a basis. Let

$$
f x_{i}=\omega_{i}^{*} \in 0^{*} .
$$

Since $D^{*}$ is the completion of the $m$-adic ring $\mathfrak{D}$, we can find elements $\omega_{1}, \omega_{2}, \cdots$, $\omega_{r}$ in 0 such that

$$
\omega_{i} \equiv \omega_{i}^{*}\left(\bmod \mathrm{m}^{* 2}\right) .
$$

Let $\zeta_{i}$ be the $\mathrm{m}$-residue of $\omega_{i}$. By $(41), \zeta_{i}$ is also the $\mathrm{m}^{*}$-residue of $\omega_{i}^{*}$. Since $\mathfrak{o}^{\prime *} / \mathfrak{m}^{\prime *}=\mathfrak{o}^{\prime} / \mathfrak{m}^{\prime}=\mathcal{F}\left(W^{\prime}\right)=\kappa\left(\zeta_{1}^{\prime}, \zeta_{2}^{\prime}, \cdots, \zeta_{r}^{\prime}\right)$, it follows from (40) that $\mathrm{o}^{*} / \mathfrak{m}^{*}=\kappa\left(\zeta_{1}, \zeta_{2}, \cdots, \zeta_{r}\right)$, that is (since $\left.0^{*} / \mathfrak{m}^{*}=\mathfrak{o} / \mathfrak{m}=\mathcal{F}(W)\right)$ :

(a) The $W$-residues of $\omega_{1}, \omega_{2}, \cdots, \omega_{r}$ generate over $\kappa$ the entire field $\mathcal{F}(W)$, Let

$$
\begin{aligned}
t_{i}^{*} & =f t_{i}^{\prime}=\phi_{i}\left(\omega_{1}^{*}, \omega_{2}^{*}, \cdots, \omega_{r}^{*}\right), \\
t_{i} & =\phi_{i}\left(\omega_{1}, \omega_{2}, \cdots, \omega_{r}\right) .
\end{aligned}
$$

Since $t_{1}^{\prime}, t_{2}^{\prime}, \cdots, t_{r-\rho}^{\prime}$ is a basis of $\mathfrak{m}^{*}$, it follows from (42) that $t_{1}^{*}, t_{2}^{*}, \cdots$, $t_{r-\rho}^{*}$ is a basis of $\mathrm{m}^{*}$. In view of $\left(42^{\prime}\right)$ and $(41)$ we have $t_{i}^{*}=t_{i}\left(\bmod \mathrm{m}^{* 2}\right)$. Hence $t_{1}, t_{2}, \cdots, t_{r-\rho}$ also form a basis of $\mathrm{m}^{*}$. Since the $t^{\prime}$ s belong to $\mathfrak{D}$, they also form a basis of $m$. We have then that:

(b) The polynomial ring $\kappa\left[\omega_{1}, \omega_{2}, \cdots, \omega_{r}\right]$ contains a set of uniformizing parameters of $W$ on $V$.

From (a) and (b) it follows, by Theorem 14, that $W$ is absolutely simple for $V$. This completes the proof of the theorem. 


\section{REFERENCES} 487.

1. C. Chevalley, On the composition of fields, Bull. Amer. Math. Soc. vol. 48 (1942) pp. 482-

2. - On the theory of local rings, Ann. of Math. vol. 44 (1943) pp. 690-708.

3. I. S. Cohen, On the structure and ideal theory of complete local rings, Trans. Amer. Math. Soc. vol. 59 (1946) pp. 54-106.

4. I. S. Cohen and A. Seidenberg, Prime ideals and integral dependence, Bull. Amer. Math. Soc. vol. 52 (1946) pp. 252-261.

5. W. Krull, Idealtheorie, Ergebnisse der Mathematik und ihrer Grenzgebiete, vol. 4, no. 3, Berlin, 1935.

6. — Zum Dimensionsbegriff der Idealtheorie, Math. Zeit. vol. 42 (1937) pp. 745-766.

7. —- Dimensionstheorie in Stellenringen, J. Reine Angew. Math. vol. 179 (1938) pp. 204-226.

8. S. MacLane, Modular fields, I, Separating transcendence bases, Duke Math. J. vol. 5 (1939) pp. 372-393.

9. F. K. Schmidt, Über die Erhaltung der Kettensütze der Idealtheorie bei beliebigen endlichen Körpererweiterungen, Math. Zeit. vol. 41 (1936) pp. 443-450.

10. B. L. van der Waerden, Moderne Algebra, vol. 2, Berlin, 1931.

11. O. Zariski, Some results in the arithmetic theory of algebraic varieties, Amer. J. Math. vol. 61 (1939) pp. 249-294.

12. - Algebraic varieties over ground fields of characteristic zero, Amer. J. Math. vol. 62 (1940) pp. 187-221.

13. - Foundations of a general theory of birational correspondences, Trans. Amer. Math. Soc. vol. 53 (1943) pp. 490-542.

14. - The theorem of Bertini on the variable singular points of a linear system of varieties, Trans. Amer. Math. Soc. vol. 56 (1944) pp. 130-140.

15. - A new proof of Hilbert's Nullstellensatz, Bull. Amer. Math. Soc. vol. 53 (1947) pp. 362-368.

UNIVERSITY OF ILLINOIS,

URbaNa, ILL. 